NBS

\title{
PUBLICATIONS
}

\section{NISTIR 88-3894}



\section{Evaluation of Colors for Use on Traffic Control Devices}

\author{
Belinda L. Collins
}

U.S. DEPARTMENT OF COMMERCE

National Institute of Standards and Technology

(Formerly National Bureau of Standards)

National Engineering Laboratory

Center for Building Technology

Building Environment Division

Gaithersburg, MD 20899

November 1988

Prepared for:

Federal Highway Administration

6300 Georgetown Pike

McLean, VA 22101

$-Q C$
100
.456
$\# 88-3894$
1988
$C .2$







\section{Evaluation of Colors for Use on Traffic Control Devices}

Belinda L. Collins

U.S. DEPARTMENT OF COMMERCE

National Institute of Standards and Technology

(Formerly National Bureau of Standards)

National Engineering Laboratory

Center for Building Technology

Building Environment Division

Gaithersburg, MD 20899

November 1988

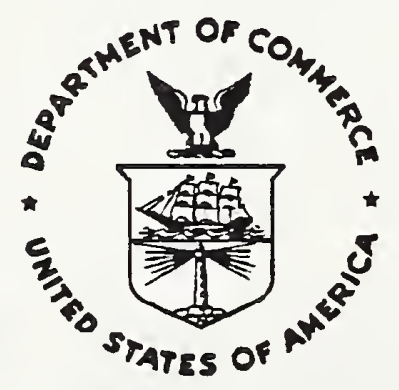

National Bureau of Standards became the National Institute of Standards and Technology on August 23, 1988, when the Omnibus Trade and Competitiveness Act was signed. NIST retains all NBS functions. Its new programs will encourage improved use of technology by U.S. industry.

Prepared for:

Federal Highway Administration

6300 Georgetown Pike

McLean, VA 22101

\section{U.S. DEPARTMENT OF COMMERCE \\ C. William Verity, Secretary}





\section{Abstract}

Color has traditionally been used to convey safety information because of its ability to attract attention and evoke a rapid response. In the present report, research on color coding, highway safety color codes, color deficiency, retroreflective materials, and conspicuity, is reviewed to evaluate the effectiveness of chromaticity specifications for highway signs and markings. The current highway specifications are typically for colors of medium lightness and saturation, which sometimes can appear quite dark. Data from a study by Collins et al. (1986) are reanalyzed to compare color appearance data for both ANSI and highway colors viewed under seven illuminants, including several HID sources. The analysis demonstrated that the ANSI colors, especially safety yellow, were identified more accurately in terms of color name, lightness, saturation, and primary hue than the corresponding highway color. In addition, selected measurements of the chromaticity of retroreflective materials were made as background for assessing the feasibility of developing a nighttime chromaticity specification. Based on the analysis of the appearance of safety colors under different illuminants, it is recommended that consideration be given to switching the FHWA specifications for the chromaticity of colors used on traffic control devices to the ANSI specifications.

Keywords:

Chromaticity, CIELAB, color, color code, color vision, color defects, conspicuity, highway, safety, retroreflectivity. 
This report was prepared under Federal Highway Administration (FHWA) contract No. DTFH61-87-Y-00073 to the National Bureau of Standards, now the National Institute of Standards and Technology (NIST), from June 1987 through October 1988.

\section{Acknowledgements}

The author wishes to acknowledge all the people who contributed to this research project including the manufacturers of retroreflective color samples who supplied the samples used in this report, the Contracting Officer's Technical Representative, Mr. Richard Schwab who provided many helpful insights, Dr. James Worthey and Mr. W.N. Hale who took many of the retroreflective color sample measurements, and Ms. Belinda Kuo and Mr. Gary Gillette who did many of the graphs. In addition, Dr. James Worthey reviewed the final document and provided important contributions, while Mr. W.N. Hale wrote Appendix A. The final report could not have been completed without the able assistance of all these people.

\section{Disclaimer}

Commercial products and tradenames are provided in this report for informational purposes only, and do not constitute an endorsement or recommendation by the National Institute of Standards and Technology or the Federal Highway Administration. 
1. Introduction . . . . . . . . . . . . . . . . . . . . . 1

1.1 Purpose... . . . . . . . . . . . . . . . . . . 1

1.2 Existing Codes and Standards . . . . . . . . . . . . . . . 2

1.3 Introductory Information on Color Research . . . . . . . . 4

1.3.1 Color Appearance . . . . . . . . . . . . . . . . . . 4

1.3.2 Color Codes . . . . . . . . . . . . . . . . . 6

2. Background Information on Color Vision . . . . . . . . . . . . . 16

2.1 Normal Color Vision . . . . . . . . . . . . . . . . . . . 16

2.2 Use of Color Coding to Convey Information . . . . . . . . . 18

2.3 Problems Associated with Defective Color Vision . . . . . . 20

2.3.1 Color Vision Defects . . . . . . . . . . . . . . . 20

2.3.2 Implications of Color Deficiency for Color Coding . . 23

2.3.3 Color Deficiency in the Transport Industry . . . . . 26

2.4 Problems Associated with Aging and Acquired Color Defects . 29

2.5 Problems Associated with the Viewing Condition . . . . . . 29

2.5 .1 Impact of the Illuminant . . . . . . . . . . . . . 29

2.5.2 Impact of the Object Color . . . . . . . . . . . . . 31

2.5.3 Effectiveness of Highway Colors . . . . . . . . . 33

2.6 Other Studies of Highway Sign Colors . . . . . . . . . . 56

3. Measurement of Retroreflective Colors . . . . . . . . . . . . . 58

3.1 Background on Retroreflection . . . . . . . . . . . . . . . 58

3.2 Measurement of Retroreflective Materials . . . . . . . . . 59

3.3 Comparison of Chromaticity for Retroreflective Color Samples 61

4. Conclusions. . . . . . . . . . . . . . . . . . . . . . 71

4.1 Feasibility of Altering Current Highway Color Specifications 71

4.2 Additional Research . . . . . . . . . . . . . . . . . . . . 71

5. Recommendations. . . . . . . . . . . . . . . . . 74

6. Bibliography . . . . . . . . . . . . . . . . . 75

Appendix A. Feasibility of Nighttime Color Specifications . . . . 82

Appendix B. CIE $x, y$ and CIELAB Specifications for Highway Colors. 98

Appendix C. Definitions . . . . . . . . . . . . . . . . . 99 
Figure 1 Chromaticity regions specified by the CIE for ordinary colors under D65.

Figure 2 Chromaticity regions specified by the CIE for retroreflective colors under D65

Figure 3 Chromaticity regions specified by the FHWA for retroreflective colors under source $C$.

Figure 4 Chromaticity regions specified by ANSI 253.1 for ordinary surface colors for source $C$.

Figure 5. Confusion lines for dichromatic observers. . . . . . . 25

Figure 6. CIELAB comparison of red samples . . . . . . . . . . 35

Figure 7. Color naming, lightness and saturation judgements for ANSI red.

Figure 8. Color naming, lightness and saturation judgements for sample \#11 (highway red)

Figure 9. CIELAB comparison data for orange samples.

Figure 10 Color naming, lightness, and saturation data for ANSI orange.

Figure 11 Color naming, lightness, and saturation data for sample \#35 (highway orange).

Figure 12 CIELAB comparisons for yellow samples... . . . . . . . 46

Figure 13 Color naming, lightness, and saturation data for ANSI yellow.

Figure 14 Color naming, lightness, and saturation data for sample \#21 (highway yellow)................ 48

Figure 15 CIELAB comparisons for green samples. . . . . . . . . . . 50

Figure 16 Color naming, lightness, and saturation data for ANSI green.... . . . . . . . . . . . . . 51

Figure 17 Color naming, lightness, and saturation data for sample \#25 (highway green).

Figure 18 CIELAB comparisons for blue samples. 
Figure 19 Color naming, lightness, and saturation data for ANSI blue. . . . . . . . . . . . . . . . . . . 54

Figure 20 Color naming, lightness, and saturation data for sample \#27 (highway blue). . . . . . . . . . . . . . . 55

Figure 21 Instrumental comparisons for engineering grade samples. . . 63

Figure 22 Shift from illuminant $C$ to illuminant A for both retroreflective and $45 / 0$ measuring conditions for engineering samples. . . . . . . . . . . . . . . . . . . 64

Figure 23 Instrumental comparisons for high intensity samples. . . . . 66

Figure 24 Shift from illuminant $C$ to illuminant A for both retroreflective and $45 / 0$ measuring conditions for high intensity samples . . . . . . . . . . . . . . . . 67

Figure 25 Shift from illuminant $C$ to illuminant A for two measuring conditions for another set of engineering samples . . . . 68

Figure 26 Shift from illuminant $C$ to illuminant A for two measuring conditions for another set of high intensity samples . . . .69 
Table 1 Chromaticity Coordinates for National and International Safety Color Codes. . . . . . . . . . . . . . 13

Table 2. Psychophysical Comparisons for ANSI and Highway Color Samples . . . . . . . . . . . . . . . . 39 


\section{INTRODUCTION}

Color has traditionally been used to convey safety information, to organize complex displays, and to create moods (Cole and Vingrys, 1985). The typical application of color for traffic control devices is that of coding safety information and directions to allow a motorist to see a colored sign and respond immediately with the desired action. Thus, red is used for stop signs, yellow for warning signs, and green for directional signs on highways in the United States (U.S).

The current specifications for highway colors (American Association of State Highway and Transportation Officials or AASHTO, M268-84; Department of Transportation (DOT) Manual on Uniform Traffic Control Devices or MUTCD, 1986) are for ten highly saturated colors plus black and white. Detailed specifications for the seven colors in current use are given in Appendix B. The color specifications given by AASHTO and the MUTCD ${ }^{1}$ are different from those given by the American National Standards Institute (ANSI) Z53.1 (1979) standards and the Department of Transportation Hazardous Materials Transport (1973) regulations, and those given in international standards such as the Commission Internationale de l'Eclairage or CIE (1983), the International Standardization Organization or ISO (1984), and the Deutsches Institut für Normung or DIN (1970). The colors specified for U.S. highways are somewhat darker, particularly the yellow, blue and green, and may not be recognized as accurately as the colors specified by the ANSI and other systems. In addition, current specifications for highway red, orange and yellow occupy only a small region of color space so that orange is too close to both yellow and red, leading to confusions, especially between yellow and orange.

\subsection{Purpose}

The purpose of the present report is to explore and evaluate the effectiveness of the chromaticity specifications for colors used on highway signs and markings. The current specifications are for highly saturated colors of medium lightness ${ }^{2}$ - which can result in some colors which are so dark as to be difficult to recognize accurately. Existing research on color and highway applications is reviewed in the present paper to determine the need for changing the chromaticity and luminance specifications to improve the conspicuity and recognizability of highway safety colors, both ordinary and retroreflective. Data from a study by Collins et al. (1986) on the appearance of safety colors are reanalyzed to compare the appearance of the ANSI colors with those specified for highway applications.

1 In subsequent references to color specifications, the term "FHWA" will be used rather than MUTCD or AASHTO, since the MUTCD is published by the Federal Highway Administration (FHWA), and since it references the AASHTO specifications.

${ }^{2}$ Except yellow which has high lightness. 
Another purpose of the present paper is a discussion of ways of measuring the chromaticity of highway signs including both laboratory and field methods. Highway signs are usually retroreflective - meaning that they reflect radiation in directions close to the direction from which the light came (American Society for Testing and Materials or ASTM E 80881). The current specifications for highway colors require that they "shall be reflectorized or illuminated to show the same shape and color both by day and night. All overhead sign installations should be illuminated where an engineering study shows that reflectorization will not perform adequately" (MUTCD 1983, p. 2A-7). Yet, no guidance is given for determining that the color is the same both by day and night. The feasibility of measuring the chromaticity of retroreflective signs in field locations is discussed in Appendix $A$ along with a presentation of a possible field procedure. Laboratory measurements of the chromaticity of retroreflective colors, using different measuring geometries and instruments are presented in section 3 .

\subsection{Existing Codes and Standards}

Use of color coding for highway traffic signs evolved only slowly into the modern code now recommended in the Manual on Uniform Traffic Control Devices (MUTCD). Thus, according to Robinson (1967), the first highway color code, developed in 1927, recommended only four colors: white, black, yellow and green (for rest stations). Red, orange, and blue were added in various revisions up to 1961, although their use was not always consistent.

The process of adding colors to the highway color code did not always run smoothly. In 1957, the noted colorist Faber Birren commented that:

"To expect average mortals to think continually in the process of seeing will seem quite contrary to human nature. In industry bright colors will mark danger spots far more effectively than will signs containing words and legends. The reason is simple enough, for visual reaction to color is involuntary, while words require deliberation" (Birren, 1957, p. 569).

At the time of Birren's article the suggestion had been made to use black as a background color for directional or guide signs. Birren objected to this proposal because black, although affording high contrast with white lettering,

"is emotionally negative, holding little visual, mental or psychologic interest" ... Green, on the other hand, is "a color which is second in recognition only to red. And because it is emotionally tranquil rather than impulsive, it would find a wholly logical and secondary sequence to red for STOP and yellow for warning or caution" (Birren, 1957, p.571).

In 1967, extensive revisions were proposed for the highway code by the National Joint Committee on Uniform Traffic Control Devices. This committee developed the following criteria for a highway color code: 1) The code should contain no more than 10 to 15 colors; 2) Presently used 
highway colors should continue to be used; 3) The separation between colors should maximize discrimination by color normal viewers; and 4) The separation between colors for color defective observers should be no worse than the worst pair, red and green, in use at the time of the revisions (Robinson, 1967).

Using these criteria, the committee selected a set of 12 colors including white, black, red, orange, yellow, green, blue, purple, bright yellow green, brown, gray, and buff, and adopted color meanings for all but bright yellow green, gray, and buff. By the time the regulation was issued in 1968, the buff color had been replaced by coral, and the gray with light blue but neither was assigned a color meaning. The following color meanings for highway colors are given in the latest version of the MUTCD (1986, revision 4):

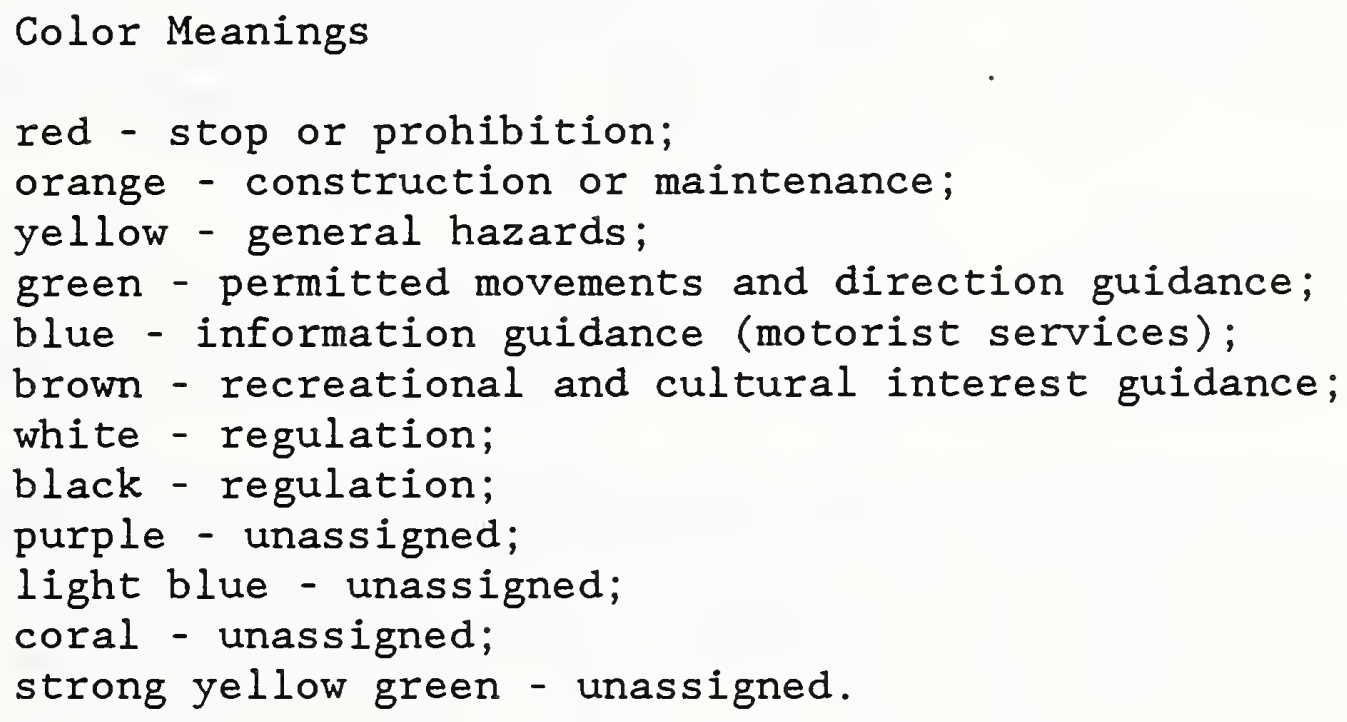

Although purple was originally selected for identifying school situations and warning of potential conflicts with school children, this meaning has since been dropped. Robinson (1967) speculated that the brown color may cause problems for some. color deficient observers who might confuse it with green or red, although evidence for this confusion has not been uncovered in subsequent research.

Robinson commented (1967, p. 29) that the recommended highway color code was an extension but not a major departure from highway practice in the $1960^{\prime} \mathrm{s}$.

"Six colors are assigned meanings entirely consistent with traditional use. Two new colors - purple and brown - are added for specific applications and another color - orange - is assigned to a new and broader use. Three other colors are identified and reserved for future uses... It is also noted that in order to provide "room" for a non-confusing orange it is suggested that highway yellow be shifted toward the yellow established for the American Standards Association Safety Color Code. Since this change is not large, it could be accomplished over a period of time without obsoleting control devices now in place on the highways" (p.28 and 29). 
This change has yet to be accomplished, however.

CIE lightness and chromaticity specifications (see p.5 for discussion) for the current highway colors as given by AASHTO are presented in appendix B. CIELAB values (see p.6) are also given in this appendix.

\subsection{Introductory Information on Color Research}

Before discussing color coding, there are a number of concepts which should be defined to aid in understanding what will follow. While the layperson uses "Color" to describe the appearance of an object, the color researcher uses two terms, "chromaticity", and "lightness". Chromaticity is a precise mathematical description of the wavelengths reflected by an object, and includes both hue and saturation, while lightness is a description of the amount of light reflected by an object. (Definitions are given in Appendix C.) Chromaticity is specified in terms of the Commission Internationale de l'Eclairage (CIE) tristimulus system in which three color primaries are used to match a given color.

\subsubsection{Color Appearance}

Perception of the color of an object is the result of the interaction of three separate variables:

1. The visual sensitivity of the observer when the object is viewed.

2. The spectral reflectance distribution of the object which is a function of the particular pigments or dyes in the object.

3. The type of illumination (spectral power distribution) under which the object is viewed.

It is easy to overlook the impact of the light source on color appearance, but as Schiff (1980, p. 35) put it,

"a red surface appears red because it selectively absorbs wavelengths other than red, and it reflects more of the long wavelengths than of those in the middle or short wavelength end of the visible spectrum. Sources, surfaces, and media that are not differentially selective in the wavelengths they send, reflect, or transmit to the eye are seen as 'achromatic' rather than colored. They appear black, gray or white."

A red object cannot preferentially reflect red (longwave) radiation if the light source does not contain long wavelength radiation, however. Commercially available sources vary widely in their spectral power distribution with low pressure sodium (LPS) containing power concentrated at about $589 \mathrm{~nm}$, and tungsten having a relatively continuous spectral power distribution. 3 The CIE uses the term "color rendering" for specifying the ability of a light source to reveal the colors of objects. The IES (1984, p.1-8) describes the Color Rendering Index (CRI) of a light source in the following manner:

${ }^{3}$ Tungsten has less power in the short wavelength portion of its spectrum than daylight. 
"a measure of the degree of color shift objects undergo when illuminated by the light source as compared with the color of those same objects when illuminated by a reference source of comparable color temperature"

The color rendering index, however, does not provide any information about an illuminant's effect on specific colors. Furthermore it is not a color appearance specification. The latter is needed to define and communicate what color a color is; that it, is the red that I see a cherry red, a burgundy red, a brick red, a magenta, or is it really pink?

A number of color appearance and specification systems have been developed so that one person can understand what another person means by the term "red" or any other color. Major systems include the Munsell system, the CIE $\mathrm{x}, \mathrm{y}$ and $\mathrm{L} * \mathrm{a} * \mathrm{~b} *$ chromaticity systems, the OSA USC color order system, the ISCC-NBS color name system, and the NCS (Swedish) color system (Billmeyer, 1987).

In the Munsell system, color is defined as a three dimensional space with three axes, hue (color), chroma (saturation) and value (lightness). The central black-white axis represents value, while the hues (colors) are arranged around this axis, with increasing chroma (saturation) as one goes further from the central axis (IES 1984; Hurvich, 1981). Color is specified by a set of numbers and letters representing hue, value/chroma (in that order). Thus, 5R 4/10 indicates a particular red, of value 4 and chroma 10. Munsell has also developed a set of 1600 color chips to represent this space.

The Inter-Society Color Council - National Bureau of Standards (ISCCNBS) system which also contains a universal color language is a color specification system using levels of color names which are defined ever more precisely as the level increases. Thus, in level one, 13 common color names define all colors, level 4 contains the Munsell system, while in level six, color is measured instrumentally, and specified in terms of the CIE chromaticity system (see Kelly and Judd, 1976). Both the Munsell and ISCC-NBS color systems are based on the use of CIE source C (or average north daylight) as the standard light source.

The Optical Society of America Uniform Color System (OSA-UCS) system is a color space with uniform visual spacing based on a rhombohedral lattice. There are three axes in this system - L for lightness, $j$ for yellownessblueness, and $g$ for greenness-redness. The OSA system is considered to be one of the best representations of human visual spacing. A set of color chips has also been developed for this space.

The Swedish National Color System (NCS) system uses a different approach for specifying color order. This system is based on the degree of resemblance between a particular color and the six elementary colors red, yellow, green, blue, black, and white. Hue is defined in terms of the percentage resemblance of the test color to the two nearest elementary colors, while chromaticness is defined in terms of the resemblance of a test color to a color of the same hue with the maximum possible chromatic content, and blackness is defined in terms of the resemblance of the color to a perfect black (Billmeyer, 1987). 
Finally, the CIE chromaticity system provides a way for specifying the color in mathematical terms for any light source whose spectral power distribution is known. This system is based on the fact that three colored lights (primaries) can be mixed to match any given color. In 1931 the color matching data obtained for the visible spectrum from a set of observers under rigorously controlled conditions were averaged and adopted as the color matching functions of the normal human eye (Standard Observer) by the CIE (Billmeyer and Saltzman, 1981; Hurvich, 1981). The amounts of the three primaries (color matching functions) needed to match a color at each wavelength are termed "Tristimulus Values" or $X, Y$, and Z. The three primaries, $\bar{x}, \bar{y}$, and $\bar{z}$, have been chosen so that they are all-positive functions.

To specify the color of an object in the CIE system, one must first determine the spectral reflectance (or transmittance) function of the object and the spectral power distribution of the light source illuminating it. These two wavelength distributions are then multiplied by each of the three tristimulus values for all wavelengths from 360-830 $\mathrm{nm}$ and summed to obtain the $\mathrm{X}, \mathrm{Y}$, and $\mathrm{Z}$ tristimulus values (Wyszecki and Stiles, 1982). The system is set up so that the chromaticity coordinates ( $x, y$, and $z$ ) are fractions (such as $x=X / X+Y+Z$ ) which sum to unity, allowing one to derive the third coordinate arithmetically if the first two are known. This approach allowed the development of the twodimensional CIE chromaticity diagram on which the $\mathrm{x}$ and $\mathrm{y}$ coordinates are plotted, and the color of an object is specified. As noted earlier, the concept of chromaticity includes both hue and saturation.

In 1978 the CIE defined two uniform color spaces - CIELUV and CIELAB. The CIELAB or CIE L*a*b* system is a mathematical transformation of the CIE system to a uniform color space which approximates the human visual response more closely by modeling additional features of the eye's response. In CIELAB, colors are plotted in comparison to white (modelling lightness and color constancy) and a nonlinearity is applied, so that equal distances between points correspond approximately to equal perceived color differences. The CIELAB space is a cube root version of the formula for evaluating color differences developed by Adams and Nickerson. Robertson (1977) compared both CIELUV and CIELAB and determined that Munsell loci of constant hue and chroma were represented somewhat more accurately in CIELAB, but neither space is completely accurate in representing uniform color differences. The CIELAB space is widely used for industrial applications such as textiles, as well as for surface colors in general.

\subsubsection{Color Codes}

There are three major types of colors used for safety alerting - ordinary surface, fluorescent, and retroreflective (as well as combinations of fluorescent and retroreflective). Ordinary surface colors are neither fluorescent nor retroreflective but rather diffusely reflecting opaque surfaces (See ASTM, 1987, p.ix). The CIE (1983, p. 2) defines fluorescent colors as materials which: 
"are paints, pigments or dyes which exhibit photoluminescence as the result of exposure to daylight. They absorb energy from daylight in the shorter wave-length regions of the visible spectrum and/or in the ultra-violet region and reradiate some of this energy at longer wavelengths, producing narrow bands of emission in the visible region."

The CIE (1983, p.2) also defines retroreflective materials as:

"differing from ordinary materials in that the light reflected from them is mainly returned in directions close to the direction from which it came. This property is maintained over wide variations of the direction of the incident light. Under diffuse incident light, e.g. daylight, the luminance factor is usually less than that obtained for corresponding ordinary colors, but may be sufficient to permit color recognition."

Retroreflective materials are available in two types - prismatic (Cube corner) and spherical lens (See ASTM F923, 1985). Spherical lens materials are available in several grades ranging from engineering to high intensity grade, (depending on the narrowness of the cone of returning light).

There are numerous color coding systems in use around the world. These include the two U.S. codes (ANSI and AASHTO) as well as two major international codes (CIE and ISO). In 1983, the CIE published recommendations for the chromaticity and luminance factor of surface colors (ordinary, fluorescent and retroreflective) to be used in visual signalling. Specification of the luminance factor (or "ratio of the specimen luminance to that of a perfect diffuser, when illuminated and viewed under specified geometry"- ASTM E 284-81) is needed for surface colors, which unlike lights, are not seen in isolation. The CIE recommendations caution that accurate perception of surface colors will depend on a number of factors, such as the spectral power distribution of the illuminant, the reflectance of the surface, the state of the observer's adaptation, and the color of surrounding areas.

For the most effective visual signalling system, the CIE recommended minimizing the number of colors. The colors that are most accurately recognized are red, yellow, green, blue, black and white with orange, purple, gray, and brown as additional colors (which should be used carefully to avoid confusions with the primary signaling colors). Additional information for traffic signs can also be rapidly conveyed by combination with contrasting color, distinctive symbols, and unique shapes. The CIE cautioned (1983, p. 4) that

"in choosing colors for a given signalling system, it is important to take into consideration the interrelations between the colors themselves and the relations between the colors and the natural surroundings of the signs. The detection of a sign, as well as the recognition of its shape, require that there should be a good contrast between the luminance and/or the chromaticity of the sign 
and its surroundings...Colors of high luminance factor may be difficult to recognize under strong illumination, e.g. yellow may be confused with white. On the other hand, colors of low luminance factor when weakly illuminated, or when seen against a light background, may be mistaken for black."

The color specifications given by the CIE have in part been chosen to be recognizable by color defective observers. The recommendations state that the purple boundary for the red chromaticity region has been chosen partly to compensate for the reduced sensitivity to the extreme red end of the visible spectrum typical of the protanopic and protanomalous observer. When green is to be used in a system with red and yellow, the CIE recommends avoiding greens on the yellow side of the color region.

In figure 1 a graph of the chromaticity regions specified by the CIE for ordinary colors (including red, orange, yellow, green, blue, purple, white, gray, brown and black) is presented, while in figure 2 a similar graph presents data for retroreflective colors (including red, orange, yellow, green, blue, purple and white). These specifications are somewhat similar but not identical to those given by ISO, and the British Standards Institute (BSI), but rather different from the DIN standard. In particular, the specifications for green vary widely among standards.

The CIE specifications differ from those used by Federal Highway Administration (FHWA) ${ }^{4}$ which also differ from those given by ANSI Z53.1 (1979). Figure 3 presents the FHWA (AASHTO) specifications while figure 4 presents the ANSI specifications. The FHWA specifications are probably least similar to those used in international practice. On the other hand, the ANSI specifications are the same as those specified by the U.S. Department of Transportation (DOT) Office of Hazardous Materials Transport and are also referenced by the Occupational Safety and Health Administration (OSHA) regulations for industrial safety (CFR, 1981).

Table 1 presents the chromaticity and luminance factor coordinates for color codes specified by different standards associations and regulatory authorities including, FHWA ${ }^{5}$, ANSI, CIE, DIN, and ISO. As both the figures and table make clear, the biggest differences occur in the green region of the spectrum with the green specified by the U.S. standards occupying a much smaller region which is shifted away from yellow toward blue. This was done to minimize red-green confusions by color defective observers (a subject which will be addressed in greater detail in section 2). Several standards, including ISO, do not give specifications for orange, while in others the yellow is shifted from red toward green.

${ }^{4}$ The FHWA references the AASHTO specifications in the Manual on Uniform Traffic Control Devices.

${ }^{5}$ The FHWA specifications are also the same as those given by the General Services Administration (GSA) in LS-300C (1977). 


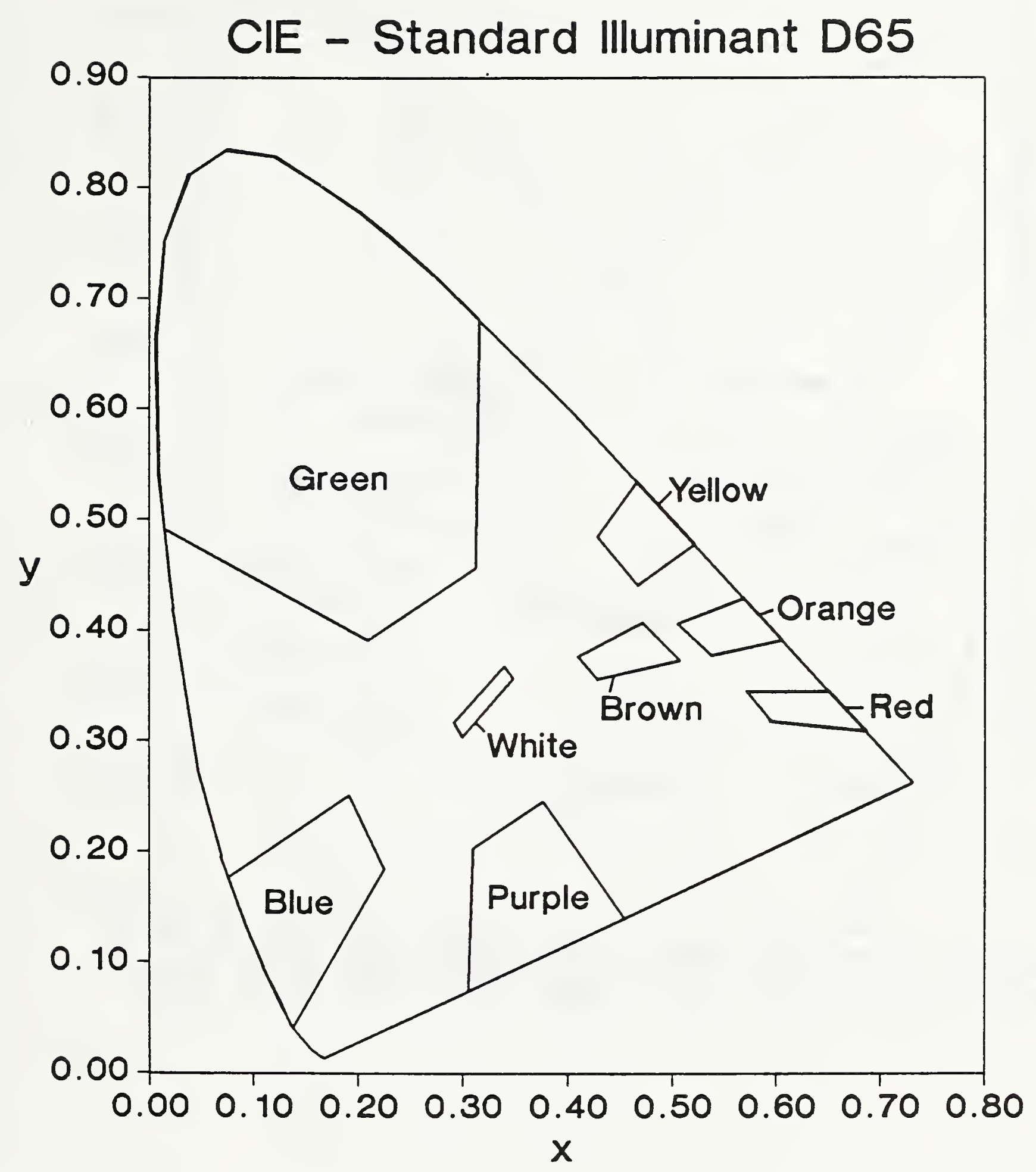

Figure 1 Chromaticity regions specified by the CIE for ordinary colors under D65. 


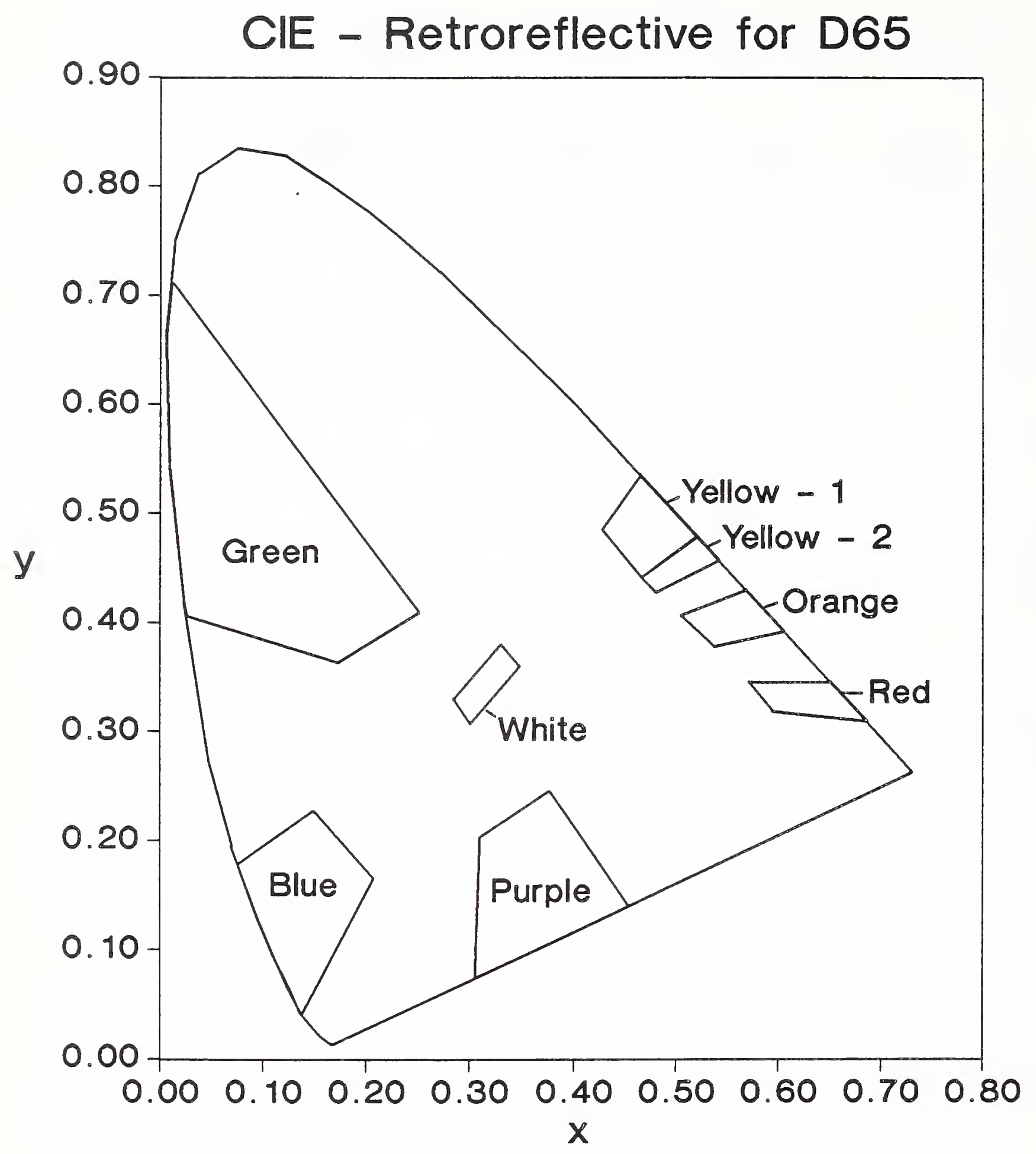

Figure 2 Chromaticity regions specified by the CIE for retroreflective colors under D65. 
FHWA III \& IV Retroreflective

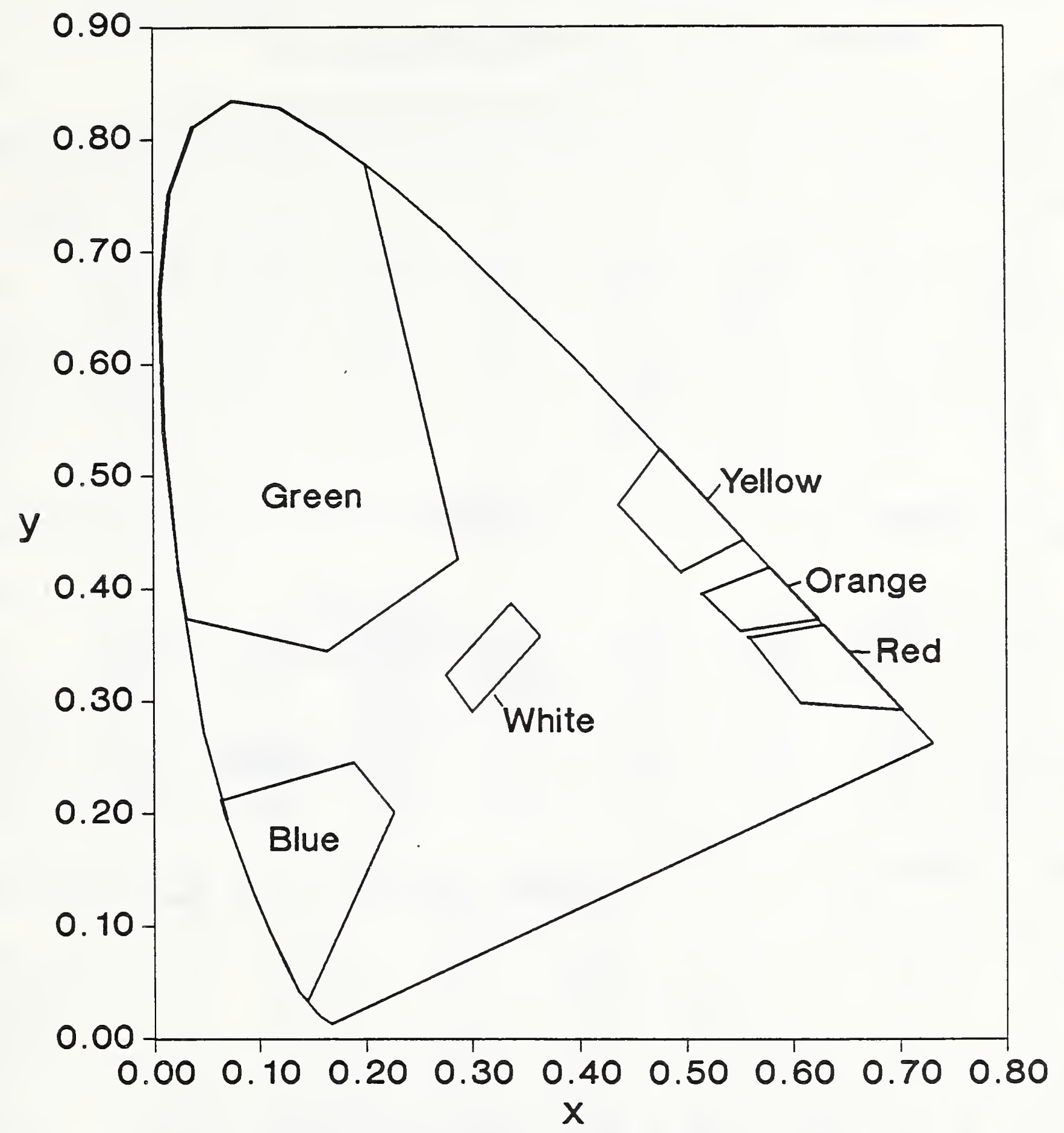

Figure 3 Chromaticity regions specified by the FHWA for retroreflective colors under source $C$. 


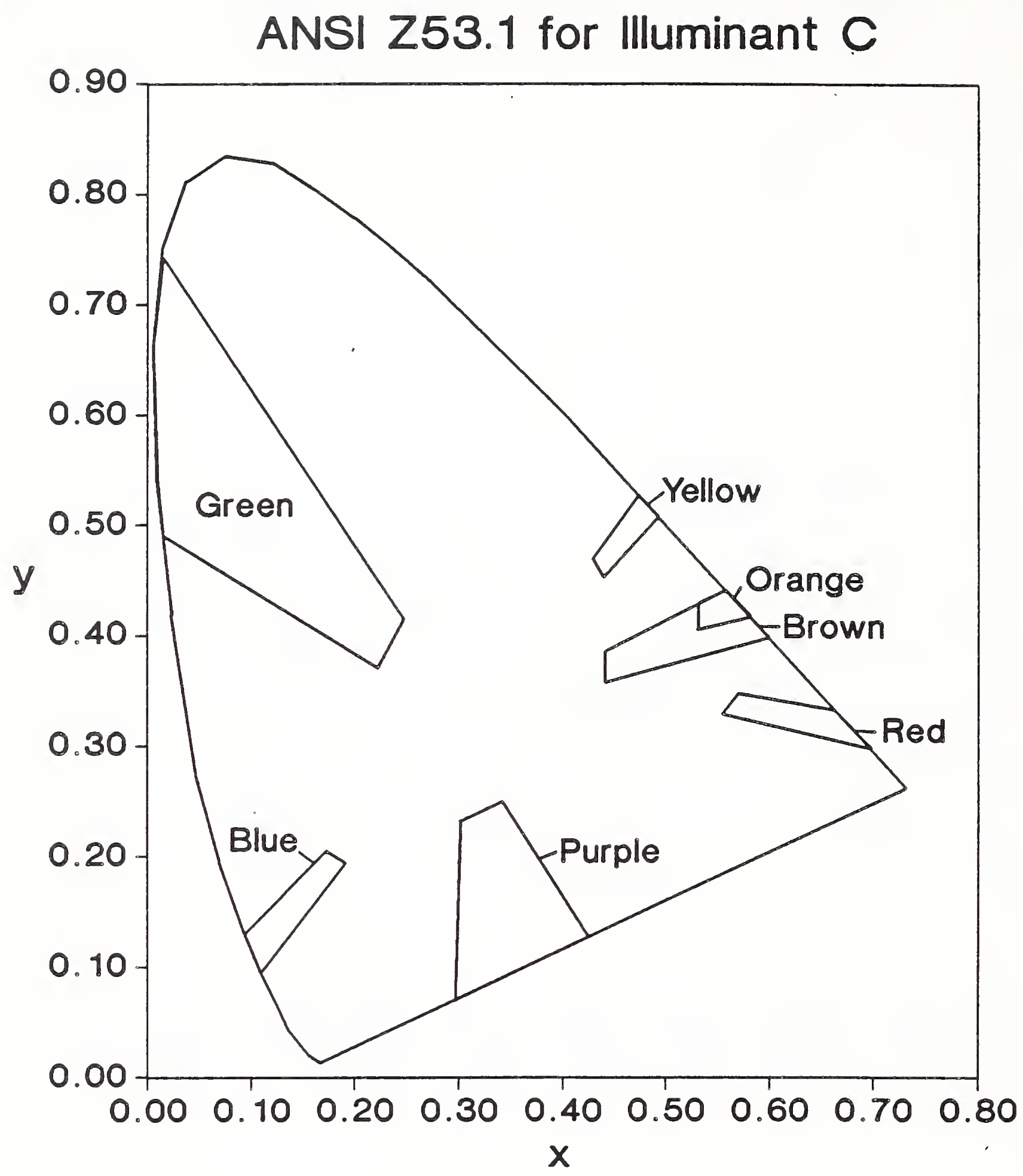

Figure 4 Chromaticity regions specified by ANSI 253.1 for ordinary
surface colors for source $C$. 
Table 1 Chromaticity Coordinates for National and International Safety Color Codes

FHWA

Types II and II-A (Engineering Grade) Retroreflective Sheeting Corner Points for Illuminant $C$

\begin{tabular}{|c|c|c|c|c|c|c|c|c|c|c|}
\hline \multicolumn{2}{|c|}{ Color } & \multicolumn{2}{|l|}{1} & \multicolumn{2}{|l|}{2} & \multicolumn{2}{|c|}{3} & 4 & \multicolumn{2}{|c|}{ y } \\
\hline & $\mathrm{x}$ & $\mathrm{y}$ & $x$ & $\mathrm{y}$ & $x$ & y & $x$ & $\mathrm{y}$ & $\min$ & $\max$ \\
\hline White & 0.305 & 0.290 & 0.350 & 0.342 & 0.321 & 0.361 & 0.276 & 0.308 & 35.0 & \\
\hline Red & 0.602 & 0.317 & 0.664 & 0.336 & 0.644 & 0.356 & 0.575 & 0.356 & 8.0 & $12 \overline{0}$ \\
\hline Orange & 0.535 & 0.375 & 0.607 & 0.393 & 0.582 & 0.417 & 0.535 & 0.399 & 18.0 & 30.0 \\
\hline Brown & 0.445 & 0.353 & 0.604 & 0.396 & 0.556 & 0.443 & 0.445 & 0.386 & 4.0 & 9.0 \\
\hline Yellow & 0.482 & 0.450 & 0.532 & 0.465 & 0.505 & 0.494 & 0.475 & 0.485 & 29.0 & 45.0 \\
\hline Green & 0.130 & 0.369 & 0.180 & 0.391 & 0.155 & 0.460 & 0.107 & 0.439 & 3.5 & 9.0 \\
\hline Blue & 0.147 & 0.075 & 0.176 & 0.091 & 0.176 & 0.151 & 0.106 & 0.113 & 1.0 & 4.0 \\
\hline
\end{tabular}

Type III and IV (High Intensity Grade) Retroreflective Sheeting for Illuminant C

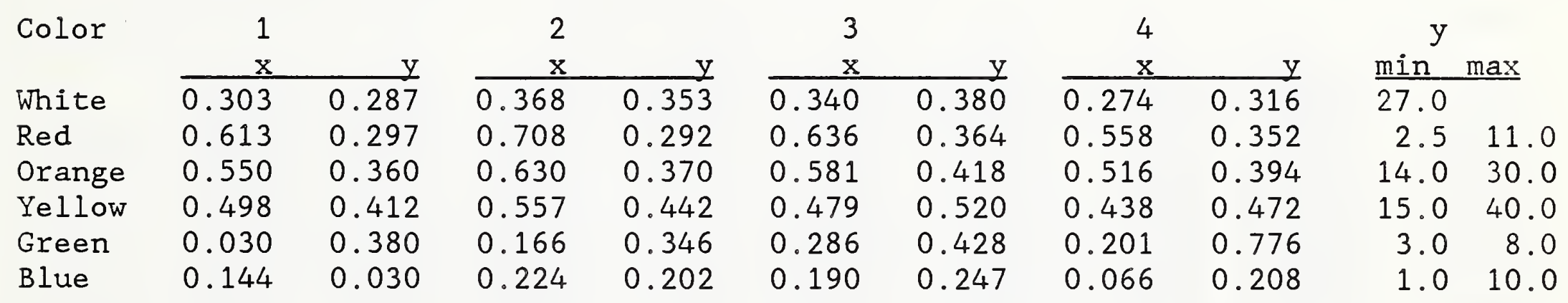

FHWA 1969 Surface Colors for Illuminant C

$\begin{array}{lccc} & x & y & Y 8 \\ \text { Red } & 0.6003 & 0.3146 & 09.00 \\ \text { Orange } & 0.5609 & 0.3955 & 24.58 \\ \text { Yellow } & 0.5007 & 0.4555 & 50.68 \\ \text { Brown } & 0.4766 & 0.3816 & 05.52 \\ \text { Green } & 0.2088 & 0.4101 & 06.56 \\ \text { Blue } & 0.1780 & 0.1833 & 06.56 \\ \text { Purple } & 0.3056 & 0.2060 & 12.00 \\ \text { Bril. Yellow Green } & 0.3461 & 00.495 & 43.06 \\ \text { Light Blue } & 0.2410 & 0.2854 & 43.06 \\ \text { Coral } & 0.3815 & 0.3169 & 51.08 \\ \text { Black } & 0.3101 & 0.3161 & 09.43 \\ \text { White } & 0.3101 & 0.3161 & 78.66\end{array}$


Table 1. Continued

ANSI Z53.1 Specifications for Safety Colors (Central Points) for Illuminant C

Color

\begin{tabular}{|c|c|c|c|c|c|c|}
\hline & $\mathrm{x}$ & y & $Y \%$ & $a *$ & $b_{*}$ & $\mathrm{~L} *$ \\
\hline Red & 0.5959 & 0.3269 & 12.00 & 56.61 & 40.98 & 41.22 \\
\hline Orange & 0.5510 & 0.4214 & 30.05 & 33.70 & 82.89 & 61.70 \\
\hline Brown & 0.4766 & 0.3816 & 5.52 & & & \\
\hline Yellow & 0.4562 & 0.4788 & 59.10 & -4.02 & 86.26 & 81.35 \\
\hline Green & 0.2110 & 0.4121 & 12.00 & -48.04 & 8.09 & 41.22 \\
\hline Blue & 0.1691 & 0.1744 & 9.00 & -0.85 & -42.23 & 35.98 \\
\hline Purple & 0.3307 & 0.2245 & 15.57 & & & \\
\hline White & 0.3101 & 0.3163 & 78.00 & & & \\
\hline Gray & 0.3101 & 0.3163 & 19.80 & & & \\
\hline Black & 0.3101 & 0.3163 & 2.02 & & & \\
\hline
\end{tabular}

Corner Coordinates for Z53.1 Specification for Safety Colors (Corner Points) for Illuminant $C$

Color

Red

Orange

Brown

Yellow

Green

Blue

Purple

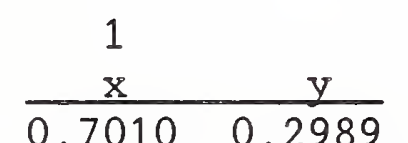

$0.7010 \quad 0.2989$

$$
0.5832
$$

0.6031

0.4162

0.3963

0.4980

0.5011

0.0129

0.0935

0.7413

0.3019

0.1273

0.6433 \begin{tabular}{cc}
2 & \\
$x$ & $y$ \\
\hline 0.5533 & 0.3240
\end{tabular}

$\begin{array}{ll}0.5533 & 0.3240 \\ 0.5331 & 0.4035\end{array}$

0.4450

0.4453

0.2445

0.1785

0.3072
0.3531

0.4492

0.4100

0.2014

0.2296 \begin{tabular}{cc}
3 & \\
$x$ & $y$ \\
\hline 0.5701 & 0.3427
\end{tabular}

0.53310 .4285

0.4450

0.4334

0.2213

0.1985

0.3509

0.3828

0.4665

0.3671

0.1930

0.2449

\begin{tabular}{cc}
4 & \\
$x$ & $y$ \\
\hline 0.6678 & 0.3320 \\
0.5606 & 0.4287 \\
0.5599 & 0.4394 \\
0.4781 & 0.5209 \\
0.0147 & 0.4851 \\
0.1079 & 0.0911 \\
0.4287 & 0.1232
\end{tabular}

Corner Coordinates for DIN 6171 Surface Colors for Traffic Signs (Corner Points) for Illuminant $C$

\begin{tabular}{|c|c|c|c|c|c|c|c|c|}
\hline \multirow[t]{2}{*}{ Color } & 1 & & \multicolumn{2}{|l|}{2} & \multicolumn{2}{|l|}{3} & \multicolumn{2}{|l|}{4} \\
\hline & $\mathrm{x}$ & $y$ & $x$ & y & $x$ & $y$ & $\mathrm{x}$ & y \\
\hline Red & 0.658 & 0.342 & 0.590 & 0.337 & 0.604 & 0.314 & 0.686 & 0.314 \\
\hline Yellow & 0.481 & 0.518 & 0.441 & 0.471 & 0.490 & 0.440 & 0.531 & 0.468 \\
\hline Green & 0.026 & 0.399 & 0.177 & 0.355 & 0.284 & 0.425 & 0.201 & 0.776 \\
\hline Blue & 0.137 & 0.038 & 0.225 & 0.180 & 0.209 & 0.226 & 0.094 & 0.125 \\
\hline $\begin{array}{l}\text { White } \\
\qquad \& \text { Gray }\end{array}$ & 0.307 & 0.307 & 0.347 & 0.347 & 0.337 & 0.357 & 0.297 & 0.317 \\
\hline White & 0.315 & 0.335 & .337 & 0.357 & 0.332 & 0.362 & 0.310 & 0.340 \\
\hline Orange & 0.583 & 0.416 & 0.533 & 0.398 & 0.552 & 0.356 & 0.631 & 0.369 \\
\hline Purple & 0.453 & 0.134 & 0.372 & 0.238 & 0.321 & 0.206 & 0.334 & 0.079 \\
\hline Black & 0.215 & 0.275 & 0.282 & 0.208 & 0.452 & 0.378 & 0.385 & 0.445 \\
\hline
\end{tabular}


CIE 1983 Surface Colors (Corner Points) for Illuminant D65

\begin{tabular}{|c|c|c|c|c|c|c|c|c|}
\hline \multirow[t]{2}{*}{ Color } & \multicolumn{2}{|l|}{1} & \multicolumn{2}{|l|}{2} & \multicolumn{2}{|l|}{3} & \multicolumn{2}{|l|}{4} \\
\hline & $\mathrm{x}$ & $\mathrm{y}$ & $\mathrm{x}$ & y & $\mathrm{x}$ & $\mathrm{y}$ & $\mathrm{x}$ & \\
\hline Red & 0.690 & 0.310 & 0.595 & 0.315 & 0.569 & 0.341 & 0.655 & 0.345 \\
\hline Orange & 0.610 & 0.390 & 0.535 & 0.375 & 0.506 & 0.404 & 0.570 & 0.429 \\
\hline Yellow & 0.522 & 0.477 & 0.470 & 0.440 & 0.427 & 0.483 & 0.465 & 0.534 \\
\hline Green & 0.313 & 0.682 & 0.313 & 0.453 & 0.209 & 0.383 & 0.013 & 0.486 \\
\hline Blue & 0.078 & 0.171 & 0.196 & 0.250 & 0.225 & 0.184 & 0.137 & 0.038 \\
\hline Purple & 0.302 & 0.064 & 0.307 & 0.203 & 0.374 & 0.247 & 0.457 & 0.136 \\
\hline White & 0.350 & 0.360 & 0.300 & 0.310 & 0.290 & 0.320 & 0.340 & 0.370 \\
\hline rav & 0.350 & 0.360 & 0.300 & 0.310 & 0.290 & 0.320 & 0.340 & 0.370 \\
\hline Lack & 0.385 & 0.355 & 0.300 & 0.270 & 0.260 & 0.310 & 0.345 & 0.395 \\
\hline rown & 0.510 & 0.370 & 0.427 & 0.353 & 0.407 & 0.373 & 0.475 & 0.405 \\
\hline
\end{tabular}

CIE 1983 Specifications for Retroreflective Colors (Corner Points) for Illuminant $\mathrm{D}_{65}$

\begin{tabular}{|c|c|c|c|c|c|c|c|c|}
\hline \multirow[t]{2}{*}{ Color } & 1 & \multicolumn{3}{|c|}{2} & \multicolumn{2}{|l|}{3} & \multicolumn{2}{|l|}{4} \\
\hline & $\mathrm{x}$ & $y$ & $x$ & $y$ & $x$ & $y$ & $\mathrm{x}$ & $\mathrm{y}$ \\
\hline Red & 0.690 & 0.310 & 0.595 & 0.315 & 0.569 & 0.341 & 0.655 & 0.345 \\
\hline Orange & 0.610 & 0.390 & 0.535 & 0.375 & 0.506 & 0.404 & 0.570 & 0.429 \\
\hline Yellow & 0.522 & 0.477 & 0.470 & 0.440 & 0.427 & 0.483 & 0.465 & 0.534 \\
\hline Yellow & 0.545 & 0.454 & 0.487 & 0.423 & 0.427 & 0.483 & 0.465 & 0.534 \\
\hline Green & 0.007 & 0.703 & 0.248 & 0.409 & 0.177 & 0.362 & 0.026 & 0.399 \\
\hline Blue & 0.078 & 0.171 & 0.150 & 0.220 & 0.210 & 0.160 & 0.137 & 0.038 \\
\hline Purple & 0.302 & 0.064 & 0.307 & 0.203 & 0.374 & 0.247 & 0.457 & 0.136 \\
\hline White & 0.355 & 0.355 & 0.305 & 0.305 & 0.285 & 0.325 & 0.335 & 0.375 \\
\hline
\end{tabular}

ISO 3864 Standard for Safety Colors and Safety Signs - 1984 (Corner Points) for Illuminant $\mathrm{D}_{65}$

\begin{tabular}{|c|c|c|c|c|c|c|c|c|}
\hline \multirow[t]{2}{*}{ Color } & 1 & \multicolumn{3}{|c|}{2} & \multicolumn{2}{|l|}{3} & \multicolumn{2}{|l|}{$r$} \\
\hline & $x$ & $y$ & $x$ & $\mathrm{y}$ & $x$ & $\mathrm{y}$ & $x$ & $\mathrm{y}$ \\
\hline Red & 0.690 & 0.310 & 0.595 & 0.315 & 0.569 & 0.341 & 0.655 & 0.345 \\
\hline Yellow & 0.519 & 0.480 & 0.468 & 0.442 & 0.427 & 0.483 & 0.465 & 0.534 \\
\hline Green & 0.230 & 0.754 & 0.291 & 0.438 & 0.248 & 0.409 & 0.007 & 0.703 \\
\hline Blue & 0.078 & 0.171 & 0.150 & 0.220 & 0.210 & 0.160 & 0.137 & 0.038 \\
\hline White & 0.350 & 0.360 & 0.300 & 0.310 & 0.290 & 0.320 & 0.340 & 0.370 \\
\hline Black & 0.385 & 0.355 & 0.300 & 0.270 & 0.260 & 0.310 & 0.345 & 0.395 \\
\hline
\end{tabular}

Brown Not Specified

Orange Not Specified

Purple Not Specified

Gray Not Specified 


\section{BACKGROUND INFORMATION ON COLOR VISION}

Current research on human color vision capabilities is discussed in the following section. The capabilities of both normal and color defective observers are documented, as well as the development of color codes for color defective observers based on their discrimination capabilities.

\subsection{Normal Color Vision}

Recently, Boynton (1988), Hurvich (1981), Jacobs (1986), Marr (1982), Pokorny and Smith (1986), Wright (1946, 1969), and Hurvich and Jameson (1972) reviewed current research in color vision. These reviews reiterated that there are two types of photoreceptors in the human eyethe rods, which operate at low illuminance levels, and the cones, which operate at higher illuminance levels and which are responsible for color perception. The rods contain one type of photopigment, maximally sensitive about $505 \mathrm{~nm}$, while it is now believed that the cone photopigments have their spectral peaks at about 419, 531 and $559 \mathrm{~nm}$ based on data from microspectrophotometry (Jacobs, 1986). The three types of color receptors in the eye are linked into an opponent color system with two separate color channels - red versus green, and blue versus yellow, as well as one achromatic channel.

The ability to discriminate color varies through the visible spectrum. Wyszecki and Stiles (1982) discussed research on the ability to make fine discriminations of differences in wavelength. They reported that all the curves for wavelength discrimination have a similar appearance, with discrimination becoming rapidly poorer at the ends of the visible spectrum. Two relative maxima of $\Delta \lambda$ are observed at approximately $460 \mathrm{~nm}$ in the blue and in the green at about $530 \mathrm{~nm}$, while three relative minima occur at approximately 440, 490, and $590 \mathrm{~nm}$. Wavelength discrimination depends on luminance level, field size, surround luminance, retinal area studied and observation technique (Pointer, 1974; Frome, Buck and Boynton, 1981; Burns and Elsner, 1985).

Generally, decreasing field size decreases discriminability, particularly if very strict foveal fixation is also used. In this case, wavelength discrimination is very poor in the blue-green region. Wright collected extensive just noticeable difference (JND) data from which it is evident that the CIE 1931 chromaticity diagram is not a uniform color appearance diagram; differences in the "green" region in the upper portion of the diagram are less detectable than similar differences in the "bluishpurple" region in the lower left portion (Wyszecki and Stiles, 1982). This means that differences in chromaticity vary through the spectrum and across mixtures of colors. Such variations are much less pronounced in CIELAB as noted earlier. Pointer (1974) found that the color temperature of the adapting field influences discrimination sensitivity, while Burns and Elsner (1985) determined that very large fields and high retinal illuminances will also alter the ability to make color matches.

Another approach to determining accurate color recognition has been the color naming approach used for surface colors. Werner and Wooten (1979) 
commented that hue naming and perception has a basis in physiology in the opponent color coding found in the ganglion and lateral geniculate cells. In a series of articles, Boynton and his colleagues explored the location of "basic" colors in the OSA set of uniform colors using three response measures - consistency of color naming, consensus of color names among subjects, and reaction times. They explored the hypothesis that certain colors are basic to the human visual system and are not a learned response specific to a particular language or culture. Thus, Boynton and Olson (1987) found that the 424 color samples of the OSA set could be largely described by a lexicon of eleven basic color terms - white, black, red, green, yellow, blue, brown, gray, orange, purple, and pink. They found a consistent use of the eleven basic terms for about $70 \%$ of the color judgements and similar consistency for $5 \%$ of the nonbasic terms. Responses for only about $5 \%$ of the judgements were both inconsistent and lacking consensus. In addition to the more consistent use of color names, response time was shortest for the basic terms. Boynton and Olson found that greens and blues tended to be used more frequently than other color names, although this may in part be due to the lack of good whites, blacks and highly saturated colors (reds in particular) in the OSA sample set. They also found that although observers agreed on color names for samples, the centroid color (calculated mathematically from the OSA chromaticity values for each color sample) for a given color name tended to be close but was not identical among observers.

Uchikawa and Boynton (1987) determined that native Japanese speakers also divided the color space into 11 categories of colors that were very similar to those used by English speaking observers. The authors "interpret the results to imply a strong physiological basis for color sensation, one that is little influenced by genetic or cultural differences between Americans and Japanese" (1987, p.1833). Boynton (1987) reported that the color naming technique works well even when the color rendering of the light source is degraded (through the addition of low pressure sodium light). He suggested that people tended to remember the name (category) of a color rather than the color sensation itself indicating that "categorical color perception, which is not tested by current color-rendering measures, is remarkably well-preserved despite illumination of very poor quality" ( $p .69$ ).

The eleven basic color names identified by Boynton and his colleagues are very similar to those used in current safety color codes. Such codes typically use white, black, red, green, yellow, blue, brown, gray, orange, and purple. (Pink is the only "basic" color not in use, although FHWA does provide for a coral color.) These data suggest strongly that current safety color coding systems are tapping into basic color sensations that observers agree upon and recognize readily.

Accurate recognition of a color depends not only on the color itself but also on the contrast with its background. In illumination engineering, contrast has traditionally been specified in terms of the luminance of a black object on a white background. Applying such logic to a colored sign does not allow the chromatic contribution to black and white 
contrast to be quantified, however. A number of laboratory studies have looked at the perceived "distinctness" of a sharp border between two areas of uniform color. These studies indicate that red-green contrast adds to black and white contrast (as an orthogonal vector) in determining the distinctness of a border (Frome, Buck and Boynton, 1981). The results from these studies are similar whether subjects assign a numerical rating to the border between the two colors; or set an achromatic border to have the same distinctness as a colored one (Frome, Buck and Boynton, 1981). Such findings would seem to have direct application to highway signs, where lettering has both achromatic and chromatic contrast with the background.

The red-green component of contrast of sign lettering depends not only on the pigments used for the sign, but also on the spectrum of the illuminant. Worthey (1982) showed that common light sources differ markedly in their ability to reveal red-green contrasts, with most fluorescent and all high intensity discharge lamps tending to reduce redgreen contrasts relative to daylight. An experiment on the distinctness of borders between colored Munsell papers revealed that illuminant differences did indeed affect the distinctness of the object-color border (Worthey, 1986b).

\subsection{Use of Color Coding to Convey Information}

Carter and Carter (1981), Christ (1975), Green and Anderson (1956) Jacobsen et al. (1985), and Vos et al. (1956) discussed the effectiveness of color coding for conveying useful information quickly and accurately, particularly under stressful conditions. Their research confirms the desirability of using color coding to convey simple, yet critically important information. Several researchers including Birren (1957), Booher (1978), Olson and Bernstein (1979), Robinson (1968), and Shinar (1985) discussed problems detecting signs, colors, and obstructions on the highway and the effectiveness of retroreflective materials for detection.

Christ (1975) reviewed and analyzed data from 42 experimental studies published between 1952 and 1973 on the effectiveness of color coding for visual displays. He found that when unique colox codes were used for a target, performance was superior for color displays relative to black and white displays. Furthermore, the increase in identification and searching performance could be as great as $200 \%$.

"More specifically, if the subject's task is to identify some feature of a target, colors can be identified more accurately than sizes, brightness, familiar geometric shapes, and other shape or form parameters, but colors are identified with less accuracy than alphanumeric symbols" (Christ, 1975, p. 560).

When color is used as a redundant variable (e.g. a particular shape is always associated with a particular color), identification accuracy for simple targets is increased when compared with the use of size or brightness as a redundant cue. A similar improvement in performance for 
color coding is found when the task is to count or locate a feature of a target in a display. Christ (1975, p. 560) commented that "compared to the use of achromatic codes, search time with the use of colors can be at least $40 \%$ less than size, $43 \%$ less than brightness, $63 \%$ less than shape, and 438 less than alphanumeric symbols. On the other hand, irrelevant colors in a display interfere with the subject's ability to locate achromatic target features". Christ found that if subjects know the color of the targets, using redundant colors can decrease search time by 32-348 for size, brightness, and shape, and 748 for alphanumeric symbols.

When Green and Anderson (1956) assessed color coding for visual search, they found that when the observer knew the target color, the search time depended on the number of symbols of the same color. When observers did not know the target color, search time was proportional to the total number of symbols in the display, thereby indicating that color can be an effective redundant code. Search time increased for multi-colored displays relative to single-color displays when observers were not told the target color. In contrast, Jacobsen, Neri, and Rogers (1985) found that the use of a fully redundant color code (using cyan, yellow, and red) resulted in significantly shorter search time (almost twice as fast) for a set of symbols when compared with a monochrome set. In addition, search time was not increased for a non-color coded dimension also present in the display. The authors concluded that color can improve performance on certain types of displays. Boynton and Bush (1956) found that increasing background complexity would also alter detectability, while Cole and Vingrys (1985) noted that road traffic signs which use redundant color coding are more likely to be located accurately and attract the motorist's attention than achromatic signs. MacDonald and Cole (1988) determined that color coding facilitated detection of warning messages that appeared unexpectedly and infrequently (such as traffic signs).

The data on color coding indicate that the use of color to provide important, easily recognized information to motorists is likely to be one of the most effective ways to ensure that they receive this information. They must, however, be familiar with the color code for maximum effectiveness. The history of color codes has involved the attempt to develop codes with colors that are maximally discriminable from each other, and immediately recognizable. Most of the research has been done with colored lights rather than surface colors, perhaps because of the need to signal information over long distances for naval and air applications.

To this end, Halsey (1959a) evaluated the discriminability of fifty different blue, green, purple and white test colors to determine the chromaticity boundaries for maximally discriminable signal lights in a color naming experiment. She found that reducing illuminance decreased the accuracy of color identification noticeably, particularly for desaturated blues, purples, and greens, with violet being frequently identified as blue. Whites were confused with yellows but not with blue or greens. Increasing the viewing distance increased observer variability and tended to shift color names slightly toward green. 
Halsey concluded that because green was identified with reasonable accuracy, the 1951 CIE boundaries for it were appropriate. Because the majority of the blues within the CIE boundaries were called "purple", she recommended that blue and purple should not be used in the same signalling system if possible.

In a second experiment, Halsey (1959b) explored the effects of removing purple as a permissible name on the accuracy of identifying blue lights. Using the same 50 test colors at two illuminance levels, she found that blue lights were identified with much greater accuracy when purple was not allowed as a choice. Again, reducing signal light illuminance resulted in poorer discrimination.

Areas requiring further evaluation include the impact of color contrast on the ability to detect sign information. As noted earlier, Frome et al. (1981) found that the distinctness of borders was enhanced when both chromatic and Iuminance contrast were present, while Worthey (1982) demonstrated that red-green contrasts (but not blue-yellow) contribute to border distinctness. Uchikawa and Kaiser (1982) demonstrated that colors which were equal in brightness and saturation were not necessarily equal in luminance. Finally, Burns, Smith, Pokorny and Elsner (1982) found that more saturated lights were brighter than less saturated lights and lights at the extremes of the spectrum were brighter than those in the middle. These experiments suggest strongly that the color of an object (or sign) can affect its perceived brightness and contrast. Since the studies were done as vision experiments using lights under carefully controlled laboratory conditions, further research is needed to extend these results to safety colors under a variety of illuminants.

\subsection{Problems Associated with Defective Color Vision}

Determining which colors are appropriate for safety color coding is complicated by the fact that some 8-10\% of the U.S. population is color defective. In the following paragraphs, the frequency of color defects will be discussed, as well as the chromaticity regions for the dominant color confusions.

Cole (1972), Cole and Vingrys (1983, 1985), Vingrys and Cole (1986) discussed the research base for existing color vision standards in the transport industries, relying on data on color confusions by different types of color defective observers and reports on accident causes. Sloan and Habel (1955a, 1955b), Nathan et al. (1964), Cole and Jenkins (1982), Halsey (1959a, 1959b), and Jameson and Hurvich (1978) reported data on color recognition by defective observers. These reviews indicated that the potential for serious confusions between some reds and greens may have been responsible for several accidents in the transport industry.

\subsubsection{Color Vision Defects}

There are two major classes of color defects - inherited and acquired. The three major types of inherited color deficiencies - anomaly, dichromacy, and monochromasy - are distinguished by the ability to match 
red and green lights. 6 As noted earlier in the discussion of the CIE chromaticity diagram, a color normal observer requires a combination of three lights (primaries) to match any given color. Although an anomalous observer also requires three primaries to match a given color, the three will differ from those used by a normal observer. Dichromatic observers require only two primaries, while monochromatic observers require only one primary. The latter essentially see the world in shades of black and white.

According to traditional classifications, defects are classified by the pigment which is missing or altered. Thus, protanomaly and protanopia refer to alteration or loss of the long wavelength pigment, deuteranomaly and deuteranopia refer to alteration or loss of the mid-wavelength pigment, and tritanomaly and tritanopia refer to alteration or loss of the short wavelength pigment. Occasionally anomalous and dichromatic defects are classified together, under the headings of "protan", "deutan", and "tritan" (Hurvich, i981).

Hurvich (1981) gave the following incidence of color deficiencies in different populations:

$\begin{array}{llll}\text { Caucasian males } & -8.08 \% & \text { Caucasian Females } & -0.74 \% \\ \text { Asian males } & -4.98 & \text { Asian Females } & -0.648 \\ \text { Other males } & -3.12 \% & \text { Other Females } & -0.69 \%\end{array}$

Hurvich also pointed out that for Caucasians the incidence of specific defects varies in the following manner:

$\begin{array}{llll}\text { Male Protanopes } & -1.0 \% & \text { Female Protanopes } & -0.028 \\ \text { Male Protanomalous } & -1.0 \% & \text { Female Protanomalous } & -0.02 \% \\ \text { Male Deuteranope } & -1.1 \% & \text { Female Deuteranopes } & -0.01 \% \\ \text { Male Deuteranomalous } & -4.98 & \text { Female Deuteranomalous }-0.38 \\ \text { Male Tritanopes } & -0.0001 \% & \text { Female Tritanopes } & -0.001 \\ \text { Male Monochromats } & -0.003 \% & \text { Female Monochromats } & -0.002 \% \\ \quad \text { Total Males } & -8.0 \% & \text { Total Females } & -0.43 \%\end{array}$

Thus, the majority of those with defective color vision are Caucasian males with roughly half of these being deuteranomalous. The few females with defective color vision also tend to be deuteranomalous.

Traditionally, an anomalous observer was considered to be color weak with less than normal sensitivity in one receptor system. More recently, anomalous observers have been considered to have one receptor response that is shifted from the normal, with the protanomalous receptor shifted toward longer wavelengths, and the deuteranomalous receptor shifted toward shorter wavelengths (Hurvich, 1972, 1981). Pokorny and Smith (1987) reported that the abnormal protan pigment peaks at about $541 \mathrm{~nm}$ as compared with the $534 \mathrm{~nm}$ peak normally found for the mid-range

${ }^{6}$ Tritan observers are identified by their inability to match a set of blue and green lights. Such observers are very rare, however. 
photopigment. The deutan peak appears to be near $553 \mathrm{~nm}$ compared with the $560 \mathrm{~nm}$ peak for the normal longwave pigment. In addition, there is a deficit in long-wave luminosity for protanomalous observers which is not found in deuteranomalous observer (Hurvich, 1972). Some anomalous observers may also have reduced neural functioning or lesser concentrations of photopigments as compared with normal observers (Pokorny and Smith, 1987). Currently, some researchers believe that most color defects are due to variations in the green pigment gene with different combinations of both hybrid and normal genes for color defective observers (Piantanida and Nathans, 1987).

Unlike the anomalous observer, who can be considered to have a shifted or anomalous photopigment, the dichromatic observer is usually considered to be missing a photopigment. Protanopic observers lack the long wavelength receptor system, deuteranopic observers lack the middle wavelength system, and tritanopes (which are very rare) lack the short wavelength system. Protanopes also have a luminosity function which is shifted toward shorter wavelengths, while both deuteranopes and tritanopes have a relatively normal spectral luminosity function. Another interpretation of the cause of the defects is that because the deuteranopic spectral luminosity function is similar to that of the normal observer, the deuteranopic photopigments are normal but the red/green opponent chromatic system has zero effectiveness (Hurvich, 1981).

A deuteranope sees only various saturations of blue and yellow, no red or green, with a white point at about $500 \mathrm{~nm}$. Because the luminosity function of the protanope is displaced toward shorter wavelengths, long wavelength light seen as yellow by the deuteranope will look darker to the protanope and may be confused with dark grays or blacks (Hurvich, 1981). For protanopes, color discrimination is absent from about $520 \mathrm{~nm}$ through the red, while for deuteranopes it is absent from about $530 \mathrm{~nm}$ through the red. Color discrimination in the tritanope is absent from about 445 to $480 \mathrm{~nm}$ (Wyszecki and Stiles, 1982). Wyszecki and Stiles (1982) noted similar reductions in color discrimination for both protanomalous and deuteranomalous observers although the reduction varies for the two types of observers. Neutral points (the point at which a monochromatic wavelength will match a given white light) for the protanope occur from 490-495 nm and for the deuteranope from 495-505 nm. The maximum of the luminous efficiency curve also varies for the various types of observers, being about $540 \mathrm{~nm}$ for protans, about, $560 \mathrm{~nm}$ for deutans, and $555 \mathrm{~nm}$ for tritans.

Detecting color defects is a difficult procedure because some observers with apparently normal photopigment systems appear to be less efficient in processing color information (perhaps at the neural level) so their color matches have greater variability than expected. Conversely, there are some anomalous observers, even some with strong deviations from normal color response functions, who have extremely good color discrimination. In fact, the view is emerging that there is a range of peak sensitivity for the visual pigments of both color normal and color defective individuals with particular variation in the long wavelength 
pigment and between males and females (Jacobs, 1987; Alpern, 1987; Mollon, 1987; Adams 1987).

In addition, color discrimination in dichromatic observers is apparently influenced by the size of the stimulus field. Such observers typically have better discrimination for larger fields and higher levels of illumination (Breton and Cowan, 1981; Boynton, 1988). Boynton (1988) pointed out that the pigment-loss explanation of dichromacy, while still adequate to explain the results for small fields, must be modified for large fields. The experimental data indicate that with large fields most dichromats can perceive red, even though they supposedly lack the receptor for red. This may be due to the presence of an anomalous third receptor activated only by the use of large fields (Boynton, 1988).

The variations in color discrimination capabilities make diagnosing color defects difficult and predicting color vision almost impossible. In addition, the picture for diagnosing tritan-type defects is complicated by the fact that changes in macular pigment and yellowing of the lens (typical of acquired color defects) also result in losses in sensitivity to short-wavelength pigments. Tests such as lantern tests (Farnsworth, Holmes-Wright), wool sorting tests (Holmgren), color chip sorting tests (Farnsworth Dichotomous Panel D and Farnsworth-Munsell $100 \mathrm{Hue}$ ), and color plate (pseudoisochromatic) tests have been developed to detect color defects. The tests seem to test different aspects of the deficiency so that a person (with a mild defect) can "pass" one test and fail another. Most of these tests will diagnose dichromatic individuals, although they may not distinguish between protan and deutan defects (Cole and Vingrys $(1982,1983)$. Using a battery of tests is the best way of diagnosing color defective observers. Unfortunately, there is no simple test for diagnosing anomalous color vision such as the pseudoisochromatic plates used for dichromats. Rather, accurate diagnosis requires use of an anomaloscope - an expensive, laboratory instrument.

\subsubsection{Implications of Color Deficiency for Color Coding}

The practical implications of color deficiency are that reds and greens tend to be confused, and protan-type observers tend to have reduced sensitivity to reds, so that reds appear very dark. Knowing that many dichromats use the color names "red" and "green" more appropriately than expected from knowledge of their visual sensitivity, Jameson and Hurvich (1978) assessed their ability to make fine color discriminations and use color names. They found that the orderings made by the dichromats varied systematically in saturation from blue colors through neutral into yellow and that as expected, protanopes appeared to use lightness differences as cues for colors. The color-naming behavior suggests that protanopes follow the rule that if a color is dark, then it should be called "red". It appears that "normal" color naming behavior occurs more frequently with protanopes than with deuteranopes because they use lightness, rather than color cues. Interestingly, Jameson and Hurvich found that although the color-naming behavior was often more accurate than expected, this did not carry over to performance of the perceptual task (namely, ordering color chips). 
The reduced luminosity of red in the protanope implies two conflicting approaches - one, that red should be made brighter to be discriminated from darker colors in the set; and two, it should remain darker to be discriminated from green. The data from various color recognition tests also suggest the need to ensure that light levels are reasonably high for safety signs and traffic lights because color discrimination appears to improve at higher levels. Booher (1978) noted the likelihood of confusions between traffic signal lights, as well as the overall reduced sensitivity to red lights, as potential problems for color defective observers.

Various color coding schemes have been proposed to improve the ability of color defective observers to detect colors. One such approach is shown in figure 5 which presents color confusion lines for different types of dichromatic observers. These lines represent sets of chromatic stimuli which will match for particular dichromatic observers. Judd, as reported by Sloan and Habel (1955a), suggested that both deuteranopes and protanopes see the visible spectrum in blue, white and yellow. As a result, he developed a color code for signal lights based on the following assumptions: 1) Dichromats who see reds as yellow will call them red if they know the color code only contains red, green, and blue; 2) Blue signals if sufficiently large and luminous will be termed blue by both dichromats and color-normal observers; and 3) Colors which vary from blue-green to yellow-green for a normal observer will appear to vary from a slightly bluish white to slightly yellowish white for a dichromat. Their low saturation will cause them to appear different than the blue and red signals in the system. If white is not included in the coding system, then this "green" will not be mistaken for it. As a result, the most feasible color code is one based on the assumption that dichromats see two distinct hues separated by a zone perceived as white.

Sloan and Habel (1955a) conducted an experimental assessment of the color code developed by Judd. Their experiment was designed to determine the minimum angular subtense and luminance of colors that could be accurately distinguished by both normal and dichromatic observers. Using eight colors at four different luminances, they attempted to ensure that the colors could not be distinguished by luminance differences alone by setting the effective luminances of red to be similar to those for the blue and bluish green. The results for ten normal observers indicated that a $1^{\circ}$ field was accurately recognized for luminances of $0.7 \mathrm{~mL}$ and greater. A similar study with 40 color defective observers indicated that all but five strongly protanopic observers identified every light correctly. If a larger test field $\left(2^{\circ}\right)$ were used, one of these five could identify the colors accurately and three others could be trained to recognize the test colors. A fifth failed the test repeatedly, however.

Sloan and Habel (1955a) commented that the fact that only protanopes experienced difficulty in distinguishing reds and greens suggests that this discrimination is intrinsically more difficult for them than for deuteranopes. The difference in the neutral points for the two types of dichromat means that the yellowish-green test lights were closer to white 


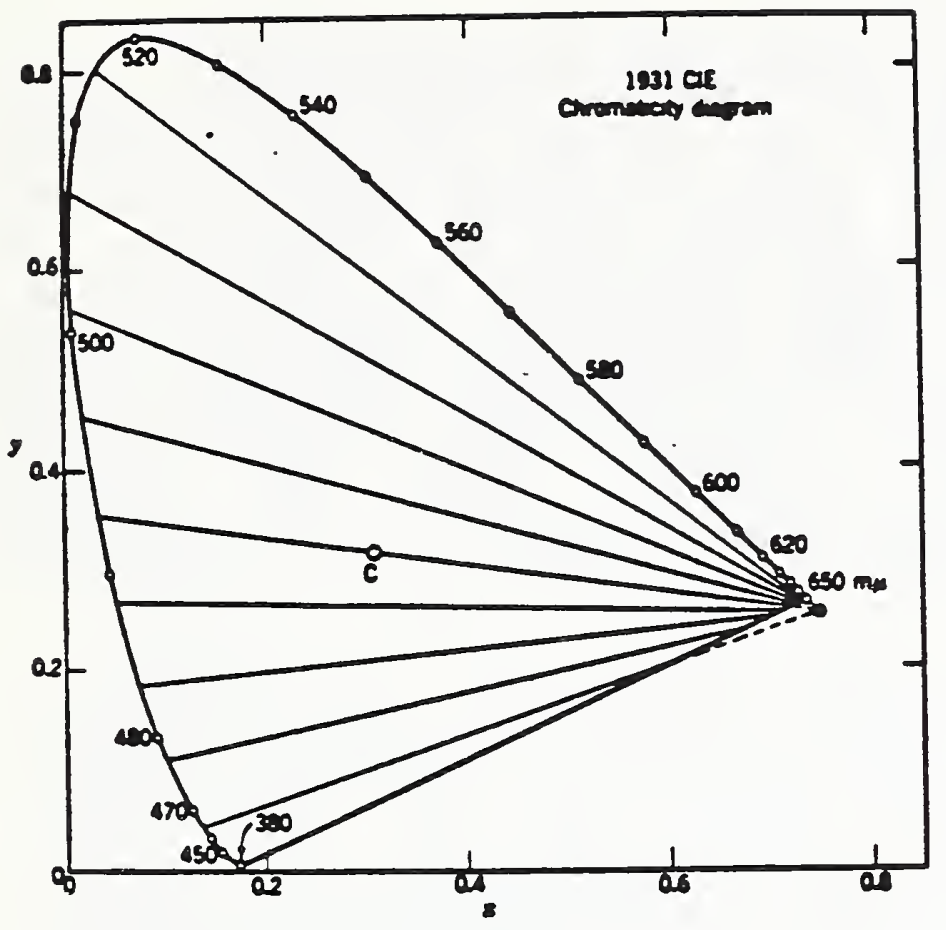

Protanopic chromaticity confusions shown on the $(x, y)$ chromaticity diagram

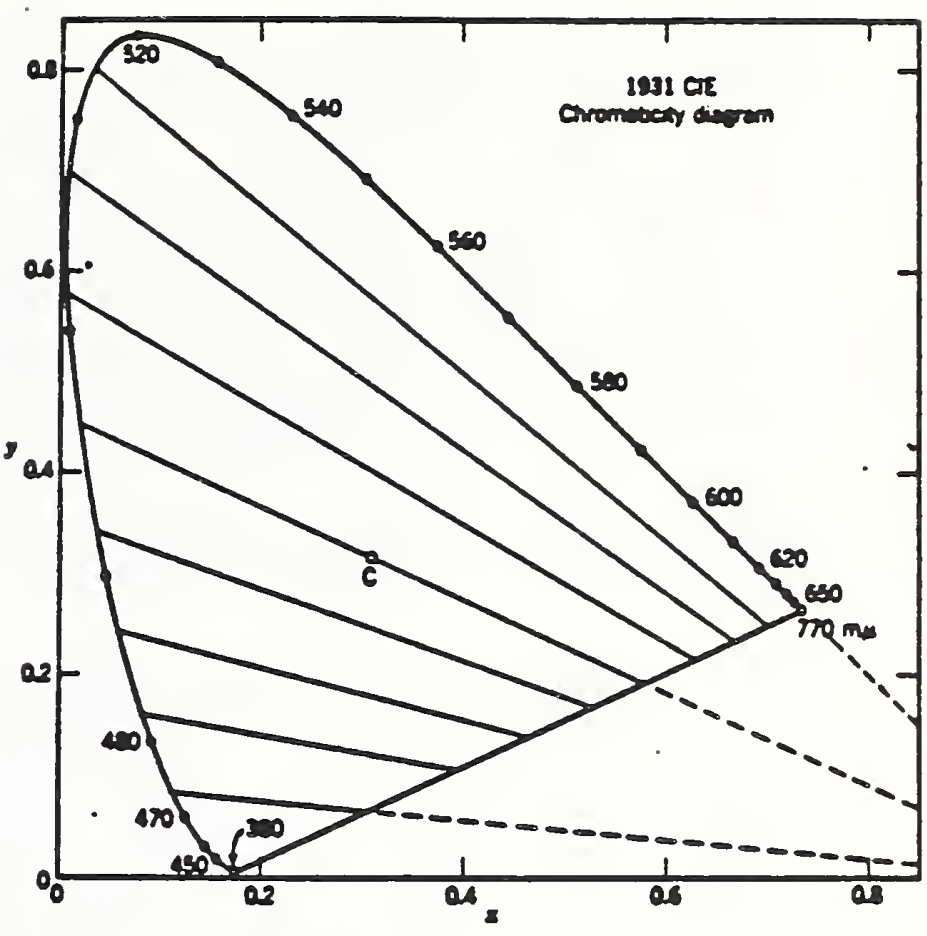

Deuteranop1c chromaticity confusions shown on the $(x, y)$ chromaticity diagram

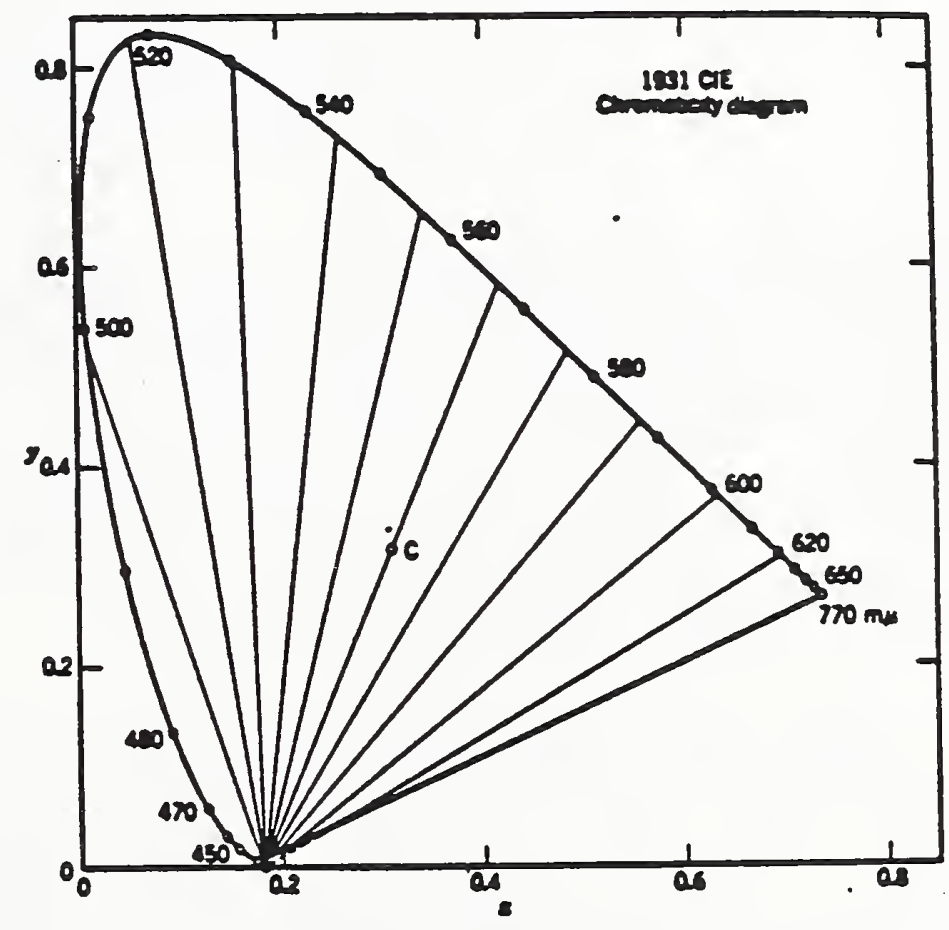

Tritanopic chromaticity confuslons shown on the $(x, y)$ chromatic1ty diagram

Figure 5. Confusion lines for dichromatic observers. Figures reproduced from Judd and Wyszecki, Color in Business. Science and Industry, 2nd Ed. (1963) with permission of John Wiley and Sons, New York. 
for the deuteranope and less likely to be confused with red. Finally, the authors suggested that protanopes also have more difficulty in using color names accurately (because perhaps they are used to using luminance cues to distinguish colors). As a result, perhaps they should be excluded from occupations requiring rapid, accurate recognition of colors. The authors noted, however, that "Even if it were necessary to exclude protanopes, the adoption of this particular three-color code would make it possible to qualify about three-fourths of those with deficient color perception" ( $p$. 598). Sloan and Habel commented that their data indicate that Judd's basic assumptions provide very accurate predictions of the chromaticity limits for distinguishable colors for most color defective observers.

In a second study, Sloan and Habel (1955b) evaluated the illuminances at which color defective observers could distinguish red and green signal lights seen at a distance, using lights subtending a very small visual angle $(0.4 \mathrm{~min})$. In this experiment, the chromaticities of the green lights were shifted slightly toward yellow (compared with the earlier experiment). The results indicated that the shift toward yellow markedly decreased a color deficient observer's ability to recognize signal colors accurately. In addition, as the intensity of the signal lights decreased, so also did the accuracy of recognition. The authors concluded that "For aviation beacons and other signals of very small angular subtense, the chromaticity limits specified by Judd must be rigidly maintained if any color deficient subjects are to use this redgreen signal system" (Sloan and Habel, 1955b, p.601).

Knowing that protanopic observers have a reduced sensitivity to red light, Cole and Vingrys (1983) determined that as the dominant wavelength of a color increases, the protanomalous response becomes more like that of the protanope, until for deep red the response is virtually the same. Both protanopic and deuteranopic observers showed a similar reduction in sensitivity to white. Their data also suggested that protanomalous observers may actually have an increased ability to detect green relative to normal observers. Decreasing signal size did not differentially decrease sensitivity to the different colors.

Cole and Vingrys (1983) calculated the reduction in visual range for protanomalous and protanopic observers and found that their visual range is about 0.4 of the normal for intense red signal colors. Protanomalous observers also have a reduction of 0.55 to 0.65 in their visual range for reds which lie within the CIE specified area for reds, and a detectable reduction in sensitivity for white lights.

\subsubsection{Color Deficiency in the Transport Industry}

Cole and Vingrys (1985) reviewed the literature on color defects, visual capabilities, and accident statistics to determine if transport standards excluding color defective operators have merit. They commented that:

"color coding is used extensively in transport systems and ... is a valuable coding dimension. It is the most useful code for long 
distance visual signalling and can facilitate accurate identification and rapid search in complex visual environments. This suggests that observers with defective color vision, who have a diminished ability to discriminate color, will be at some disadvantage in acquiring visual information quickly and accurately, either as monitors or as operators within transport systems.

The results of the experimental trials are conclusive: they all indicate that color defective observers will make significantly more errors and have significantly slower reaction times recognizing color signal lights when compared to normal observers." ( $p .7)$

Three areas of difficulty typically are cited: increased reaction time; increased number of identification errors or confusions; and failure by protan observers to see reds as reds. Thus, reaction times of 42-98\% longer than normals have been reported for color defective observers, with protanopic observers being the slowest. Error rates, too, are greater, with errors occurring most frequently for orange-red, red, yellow, green and white colors. The authors suggest that the orange red currently recommended for signal red by the CIE is too yellow and will not be recognized as red by color defective observers.

If the color code is restricted to three colors (red, green, and blue), color defectives have little difficulty with green, but if more colors are present in the code, then error rates may run as high as 40-50\%. As a result, Cole and Vingrys reiterated the notion that greens should be bluish green rather than yellowish green to avoid confusion with reds. They also noted that blues and deep reds seem to be reliably identified by all observers, and that in almost every study, protan observers made more errors than deutans but anomalous observers did not necessarily make fewer errors than trichromats. Cole and Vingrys commented that the loss of luminosity is about the same for both protanopic and protanomalous observers, which means that both should display reduced sensitivity to yellow and white lights as well as to red. The result is that the relative visual range for these observers is calculated to be between 30 528 for red signals, $84 \%$ for yellows, and $82-96 \%$ for whites. Furthermore, the recognition distances for protan observers for red vehicle lights and traffic lights are about 50-70\% of the normal observer's. Finally, protanopes require almost four times the intensity for red signals such as those used for road traffic signals (Cole and Vingrys, 1985). Many red signals and signs are likely to be ineffective for protan observers, particularly at night (Cole, 1972). The practical implication is that protan observers will fail to see red signals that are readily recognized by normal observers, or at the very least, they will have longer reaction times.

Cole and Vingrys (1985) commented that even though the laboratory data predict that color defective observers should have problems with color codes using three or more colors, this finding does not always hold up in field experiments. Nevertheless, they reviewed data from six field studies which indicated that color defectives made more errors identifying colored signal lights than did normal observers. The analysis indicated further that increasing viewing distance decreased the 
accuracy of color naming for the color defective observers. The authors concluded that the field trials indicated that color defective observers make significantly more color confusions and have a shorter visual range than normal observers.

Cole and Vingrys then examined the literature on transportation accidents to determine the number attributable to defective color vision. They noted that a number of anecdotal accounts attribute several railway accidents in England and Sweden in the late 1800's to defective color vision and consequent failure to recognize colored signal lights. Unfortunately, there is no definitive evidence of the kinds of color defects or their role, if any, in these accidents. Examination of six studies of highway accidents also indicates confusion about the type and degree of defect and its contribution to the accident. Cole and Vingrys concluded that one study apparently indicated that protans had almost double the number of rear-end collisions, while deutans had twice as many accidents at traffic-light controlled intersections. Two aviation studies indicated increased frequency of accidents in general aviation that were attributable to color deficiency, although there is some question about whether the number of flights was equal for both normal and for color defective pilots. Cole and Vingrys concluded, however, that defective color vision can be a significant risk factor in aviation.

"Although a number of road accident studies report that the color defective population as a whole is not more likely to be involved in accidents than is a normal control group, there is good evidence to suggest that protans have higher road accident rates than would normally be expected (Verriest et al., 1980). In aviation, those color defective observers who are identified as having the more severe defects of color vision (fail lantern tests) have also been shown to have a higher accident rate than a group comprised of normals and those who pass a lantern test." (p. 20)

Cole and Vingrys commented that "because of redundancy and system elasticity only rarely should a failure to appreciate a color code be critical to the operation of the system. It is likely to be critical only when visual information is degraded, as it is under adverse weather conditions, or when other elements of the system are not functioning correctly" ( $p .19$ ). They concluded that:

"clearly there is sufficient evidence to warrant the retention of color vision standards in those transport industries where the highest standards of safety are expected. It has been established that color defective observers have difficulty in the recognition of color codes and that this difficulty may lead to higher accident rates for some color defectives. Therefore, while the setting of any standard is, to a degree, arbitrary, the available data suggest that protans and those observers with the more severe defects of color vision may well be at a greater risk of accident than are other observers. (Cole and Vingrys, 1985, p. 20). 
The review of the literature on color defects indicates that current color codes have been developed to minimize red-green confusions, typically by shifting the green toward blue. This shift appears to be the most effective way to ensure that the majority of color defective observers will recognize colors at least somewhat accurately, while maintaining accurate recognition for normal observers.

\subsection{Problems Associated with Aging and Acquired Color Defects}

Problems of accurate color recognition due to the ageing process are believed to be due to yellowing of the lens and macular pigments. Both are particularly common as one ages and result in tritan-like defects. Pokorny and Smith (1986) noted that acquired color vision defects may arise from progressive cone degeneration; optic nerve disorders; choroidal disorders; pigmentary degeneration, vascular disease, glaucoma, cataract or dominant optic atrophy. They pointed out that Lakowski published a seminal paper indicating that color discrimination loss is also age-dependent, due in part to yellowing of the lens and possible sensory/performance decrements. Wright (1969) noted that the lens of the eye yellows with age which causes significant variations in color vision. In addition, variability in color discrimination between observers may arise because of variations in the density of (yellow) macular pigment. The net effect is to reduce the blue and violet energy reaching the photoreceptors for those with heavy pigmentation. Recent investigations into acquired color defects have centered on the question of possible increased sensitivity of short wavelength sensitive cones to acquired damage (Pokorny and Smith, 1986).

Although current color vision standards do not consider changes in color vision due to aging, this may not be a practical problem since the changes do not result in significant red-green confusions. Rather the losses are generally in the blue or short wavelength portion of the spectrum, which is less widely used for safety alerting.

\subsection{Problems Associated with the Viewing Condition}

The impact of variations in observer sensitivity on the detectability of colors has been discussed in great detail in the preceding section. This review pointed out the need to consider observer sensitivity in setting chromaticity standards for safety colors. While the ability to detect safety colors is influenced by the size of the sign, the overall illuminance and luminance, the background characteristics and clutter, the largest impact may be that of the illuminant. There are two other factors to be considered in this decision, however - the illuminant and the object color itself. The impact of variation in the spectral power distribution of the illuminant and in the spectral reflectance distribution of the object itself will be discussed in this section.

2.5.1 Impact of the Illuminant

The switch to High Intensity Discharge (HID) sources with higher energy efficiencies but poorer color rendering capabilities has created major 
difficulties in the accurate recognition of highway and other types of safety signs. Thornton (1977) and Jerome (1977) discussed the effect of changes in the illuminant on the recognizability of the safety colors, while Glass et al. (1983) and Collins et al. (1986) reported data on the detectability of ordinary surface, retroreflective, and fluorescent safety colors under different illuminants.

As background, Terstiege (1980) suggested that colors of transilluminated signs by day and by night should agree with the recommendations of CIE 39 (1983) as long as fluorescent lights are used, with no shift out of the designated chromaticity regions. He did not, however, evaluate possible chromaticity shifts due to HID lamps.

Jerome (1977) reported a study on the identifiability of the ANSI safety colors under different light sources including HID sources. The ANSI safety color specifications given in table 1, include red, orange, yellow, green, blue, purple, black, which are gray, and white. Jerome's experiment was conducted under quite low levels of illuminance (5.3 lux) to simulate emergency viewing conditions.

Of particular interest for highway applications are Jerome's data for fluorescent (since it is the closest to actual daylight), clear mercury, and high pressure sodium (HPS) light sources. For fluorescent, the only confusion was that $15 \%$ of the observers saw black as blue. For clear mercury, the major confusions were the following: red was termed purple by $10 \%$ and black by another $13 \%$; orange was termed red by $22 \%$ and yellow by $40 \%$; yellow was termed white by $25 \%$; green was termed blue by $18 \%$ and gray by 13\%; blue was termed black by 20\%; black was termed either blue or purple by $20 \%$ each; and both gray and white were termed yellow by $20 \%$ each. For HPS, the major confusions were as follows: red was termed orange by 198 , orange was termed red $17 \%$ and yellow 698; yellow was split evenly between yellow and white; green was termed blue by $50 \%$ and black another by $25 \%$; blue was termed black by 448 , while black was termed blue by $28 \%$. Finally gray was termed yellow by 228 , while 178 also termed white yellow. Thus, there were major confusions for the HID sources between red, yellow, and orange, and to a lesser extent between blue, green, and black, as well as between yellow, white, and gray. Under metal halide, yellow was never confused with red or orange (and vice versa), while green and blue were never confused with black. Jerome suggested that colors must be separated by at least 40 units in color space to be discriminable from each other. The effect of changing light source is to alter the separation in space between colors. He also noted that blue and green were confused with each other under all the light sources to a greater or less extent (although red and orange were confused somewhat under all sources except fluorescent).

Thornton (1977) evaluated the design of safety colors to make them more discriminable under different light sources. He pointed out that an isolated safety color must be readily identifiable as such to convey the intended information. "For reliability of identification of the safetycolor - that is, to be sure the human observer gets the proper message intended by use of the safety-color - it is essential that the color 
perceived by the observer be the intended color, and be instantly identifiable by him, by name" (Thornton, 1977, p. 92). The problem is that certain lamps such as those studied by Jerome (1977) distort the colors so that they lose their immediacy of identification. There are, of course, two solutions to the problem: one, to change the light source; and the other, to change the color. Thornton addressed the latter approach. He noted that the chromaticity of red, orange, and yellow shift toward each other under HPS and that their dominant wavelengths are in the region typically termed orange yellow. He stated further "that under the HPS lamp the gamut of coloration has become small; it follows that the safety-colors are perceived as pale or unsaturated and grayish, and this is in accord with observation. The red safety color is not identifiable as 'red'" (p. 94). Similarly, under clear mercury the dominant wavelength of ANSI Red shifts to orange and the saturation to low.

Thornton's suggestion was to redesign the spectral reflectance of the safety colors by suppressing the amount of blue-green and yellow reflectance in each color. This approach is effective for those illuminants which have spectral power distributions across the visible spectrum. Illuminants such as low pressure sodium, clear mercury, and high pressure sodium, however, have little or no energy in the red portion of the spectrum, meaning that they cannot render red (and orange) colors accurately. In these cases, the addition of fluorescence to a safety color may make it more identifiable. Thornton demonstrated theoretically that the addition of fluorescence to red, orange, yellow, and purple improved their dominant wavelength under clear mercury. The improvement was less marked for blue and green (which had maintained a more correct dominant wavelength anyway). Addition of fluorescence to red and orange for LPS is effective since their dominant wavelength is greater than $589 \mathrm{~nm}$ (the primary wavelength of LPS). A similar procedure for green and blue is not effective for LPS, however, since their dominant wavelength is below $589 \mathrm{~nm}$. Although Thornton did not use human observers in a strict experimental protocol, he reinforced the idea that the ANSI safety colors are unlikely to be identified accurately under many common lights sources, including those used in highway applications. He also suggested that these illuminants will desaturate colors by altering both their chromaticity and luminance.

\subsubsection{Impact of the Object Color}

Two studies at NBS (Glass, Howett, Lister, and Collins, 1983; and Collins, Kuo, Mayerson, Worthey, and Howett, 1986) explored the identifiability of ordinary, fluorescent, and retroreflective colors under a variety of light sources.

The first study by Glass et al. (1983) concentrated on red and orange samples in a pilot laboratory experiment which used seven observers and five light sources including tungsten, metal halide (MH), fluorescent, high pressure sodium (HPS), and low pressure sodium (LPS). Four series of samples were studied: the ten ANSI samples; 20 ordinary samples (primarily red); ten retroreflective or retroreflective fluorescent 
fluorescent samples (three yellow-green, three orange and eight red). Observers viewed each sample under each light source and responded with a color name. Responses were tabulated for the following color names: red, orange, yellow, green, blue, purple, white, brown, gray, and black, as well as two combination categories of red-orange and orange-red.

The data indicated that ANSI blue, green, and purple were generally identified correctly under all sources except LPS (although green was frequently termed blue under HPS). Yellow was correctly identified under all sources, but serious confusions occurred for orange and red under all sources except tungsten and fluorescent. For the 20 ordinary samples, two samples, a yellow-green and a red, were identified correctly more frequently than the corresponding ANSI color with all other colors having significant confusions for most illuminants. Nominally red samples were termed red, orange, or yellow depending on the illuminant. In the retroreflective series, three samples, blue, green, and orange, were more accurately identified under all light sources than the corresponding ANSI color. None of the retroreflective red samples were effective, since all had marked confusions with orange. In addition, the white retroreflective sample (viewed in a non-retroreflective mode) was confused with gray under all sources except LPS where it was termed yellow. Two fluorescent samples were identified as being effective reds with few confusions with orange or yellow even under LPS. The green fluorescent samples were effective except under LPS where they were termed yellow - a potentially dangerous confusion. The authors concluded that there is a set of colors (including red, orange, yellow, green and blue) which are more likely to be accurately identified than the ANSI set and which should be researched further.

In the second study, Collins et al. (1986) evaluated the appearance of fifty eight color samples including eleven red, ten orange, eight yellow, ten green, six blue, five purple/magenta, two brown, four white, one gray and one black. The ten ANSI samples were included as well as the "best" blue, green, orange, and red samples from the study by Glass et al. (1983). A total of 16 ordinary, 17 retroreflective, 17 fluorescent, and eight retroreflective and fluorescent samples were used. In eleven cases a particular sample was available in both a retroreflective and nonretroreflective version (with the non-retroreflective version being either ordinary or fluorescent). Each sample was studied under each of seven illuminants - tungsten (TUN), cool white fluorescent (CW), metal halide (MH), clear mercury (MER), high pressure sodium (HPS), low pressure sodium (LPS) and an equal (in illuminance) mixture of metal halide and high pressure sodium (MIX). In addition, the spectral reflectance distribution of all samples was measured under an incandescent source. The spectral reflectance distribution for each nonfluorescent sample was then calculated for the seven illuminants used in the study and the three CIE reference illuminants ( $A, C$, and $D_{65}$ ). Each of the 27 fluorescent samples was directly measured under each of the seven illuminants.

Ten color normal observers viewed each sample twice under all sources. observers reported the dominant color name, primary hue, secondary hue 
and percentage of secondary hue (if any), lightness and saturation for each sample. The results are in line with those of previous experiments which indicated serious confusions between ANSI red, orange, and yellow for LPS, HPS, and mercury, confusion of green with blue-green under HPS, and non-recognition of green under LPS. In addition, Collins et al. determined that there was a set of "best" colors which were identified more accurately under the seven light sources than the ANSI colors. This set included the fluorescent red (57) identified by Glass et al., a new fluorescent orange (48), and three ordinary colors for blue (28), green (26), and yellow (22). The ANSI samples for purple, brown, and white performed better than the comparison samples.

Collins et al. converted the spectroradiometric data for each sample into chromaticity and luminance data in both CIE $\mathrm{x}, \mathrm{y}$ and CIELAB coordinates. The data for both the ANSI and "best" samples were plotted in CIELAB space to examine the gamut of coloration under each light source. This analysis demonstrated that the gamut for the "best" colors was larger for all light sources than that for the ANSI colors, thereby supporting the contention that these colors are better representations of the safety colors.

Of interest to the present review is a comparison of the color naming results for retroreflective and non-retroreflective versions of the same sample. This indicated that in no case was the retroreflective sample identified correctly more frequently than the non-retroreflective one, and in three of the cases the performance was significantly poorer (according to a $\chi^{2}$ comparison).

\subsubsection{Effectiveness of Highway Colors}

Although the research by Collins et al. (1986) focused on the ANSI safety colors and attempts to improve their recognizability, their data represent one of the few evaluations that contained colors similar to the current highway colors. For the present report, their data were reanalyzed to determine those colors that were similar to the current FHWA colors and performance for these colors was compared with that for the ANSI safety colors for each of the seven illuminants. Because data from this study were obtained by viewing color samples under diffuse illumination conditions, the experimental conditions simulate signs illuminated by overhead illumination or daylight. They do not simulate viewing signs under nighttime highway conditions in which signs are illuminated directionally by car headlamps.

Similarity between the FHWA colors and the experimental colors was determined by comparing each of the 58 samples visually with the appropriate set of colors in the Highway Color Tolerance Charts and then graphing those colors which were a reasonable visual match in CIELAB space. Examination of the CIE and CIELAB specifications given in Appendix b, indicated that a number of the (retroreflective) colors used in that experiment, specifically red \#11, orange \#35, yellow $\# 21$, green \#25, and blue \#27, were very similar to those specified by FHWA for highway use. In subsequent paragraphs, these samples will be referred to 
as "highway" colors since they are close to, but not always identical with, the FHWA centroid color specifications.

In the reanalysis, the psychophysical results (for dominant color name, primary hue, lightness and saturation) were examined for both the ANSI standard color and the color most similar to the current highway specifications for red, orange, yellow, green, and blue. A color sample was considered to be a good example of a particular color if it had a high percentage of that color name as the dominant color name, medium lightness (except for yellow which should have high lightness), high saturation, and a high percentage (above 90\%) of the desired color as the primary hue.

Figure 6 presents two graphs of CIELAB data for ANSI red, FHWA red and two retroreflective red samples (\#11 and \#33) studied by Collins et al. (1986). These graphs present two views of data which are, in effect plotted in the three-dimensional $L * a * b *$ space. In the upper graph, the data are presented in an $a * b *$ chromaticity space, in which a* represents the red-green dimension and $b *$ represents the blue-yellow dimension. In the lower graph the data are presented in a L*a* space in which L* represents lightness. Inspection of the two graphs in figure 6 indicates that sample \#11 is very close in both chromaticity and lightness to highway red, confirming the visual comparison with the color tolerance charts.

The color naming, lightness, and saturation data for ANSI red are shown in figure 7 for each of the seven light sources studied, while similar data for sample 11 (highway red) are shown in figure 8. The top portion of each figure presents the percentage of times that a particular color name was given for the sample. The lower left figure presents the data for percentage lightness (high, medium and low), while the lower right presents the data for percentage saturation (again, high, medium and low). Comparison of figures 7 and 8 indicates that highway red (\#11) was termed red more frequently than ANSI red under all sources except HPSwhere it was never termed red. Highway red (\#11) was also seen as having medium lightness and high saturation more frequently than ANSI red. 

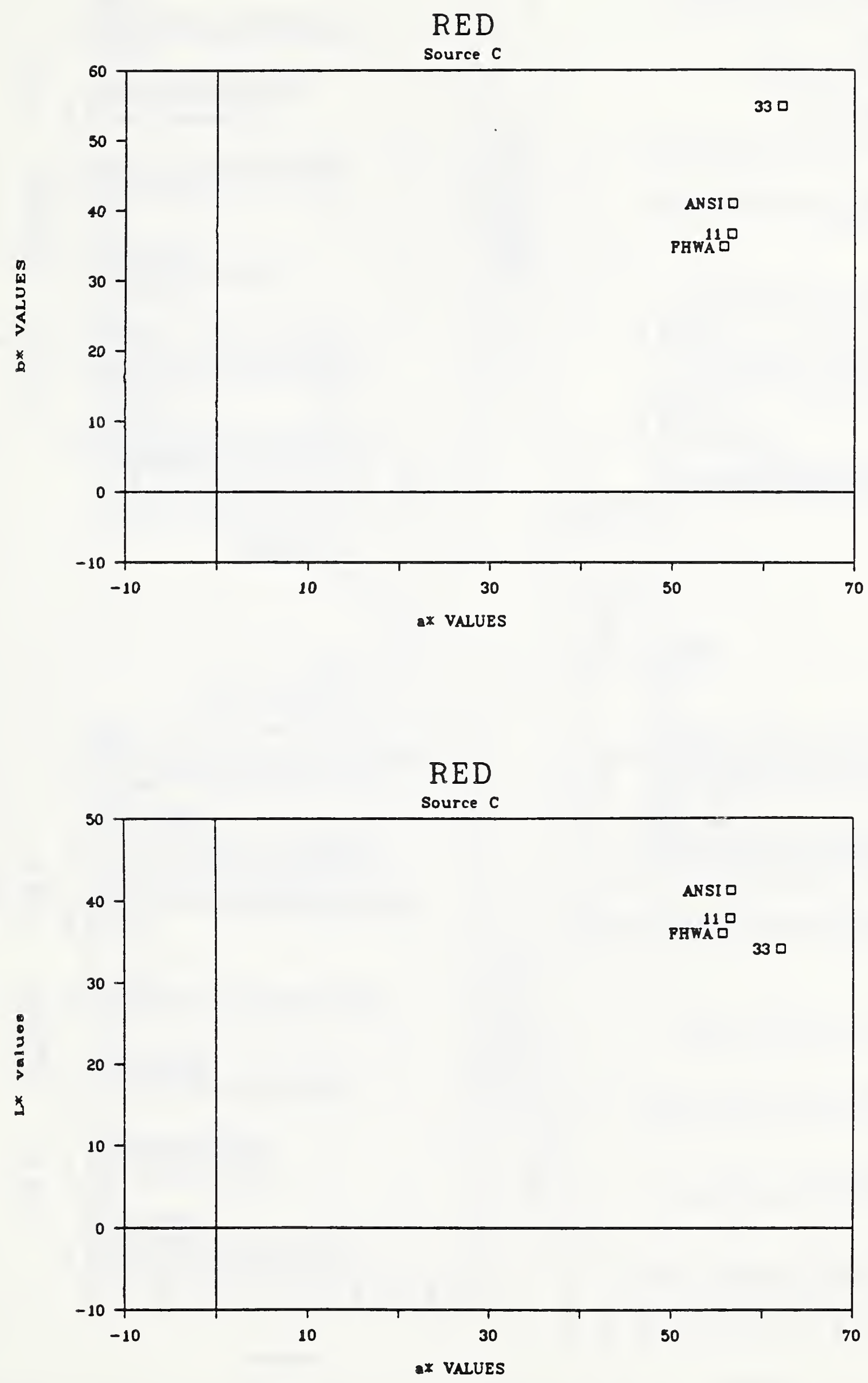

Figure 6. CIELAB comparison of red samples. The upper graph presents b* as function of a* while the lower graph presents $b *$ as a function of $L *$. 

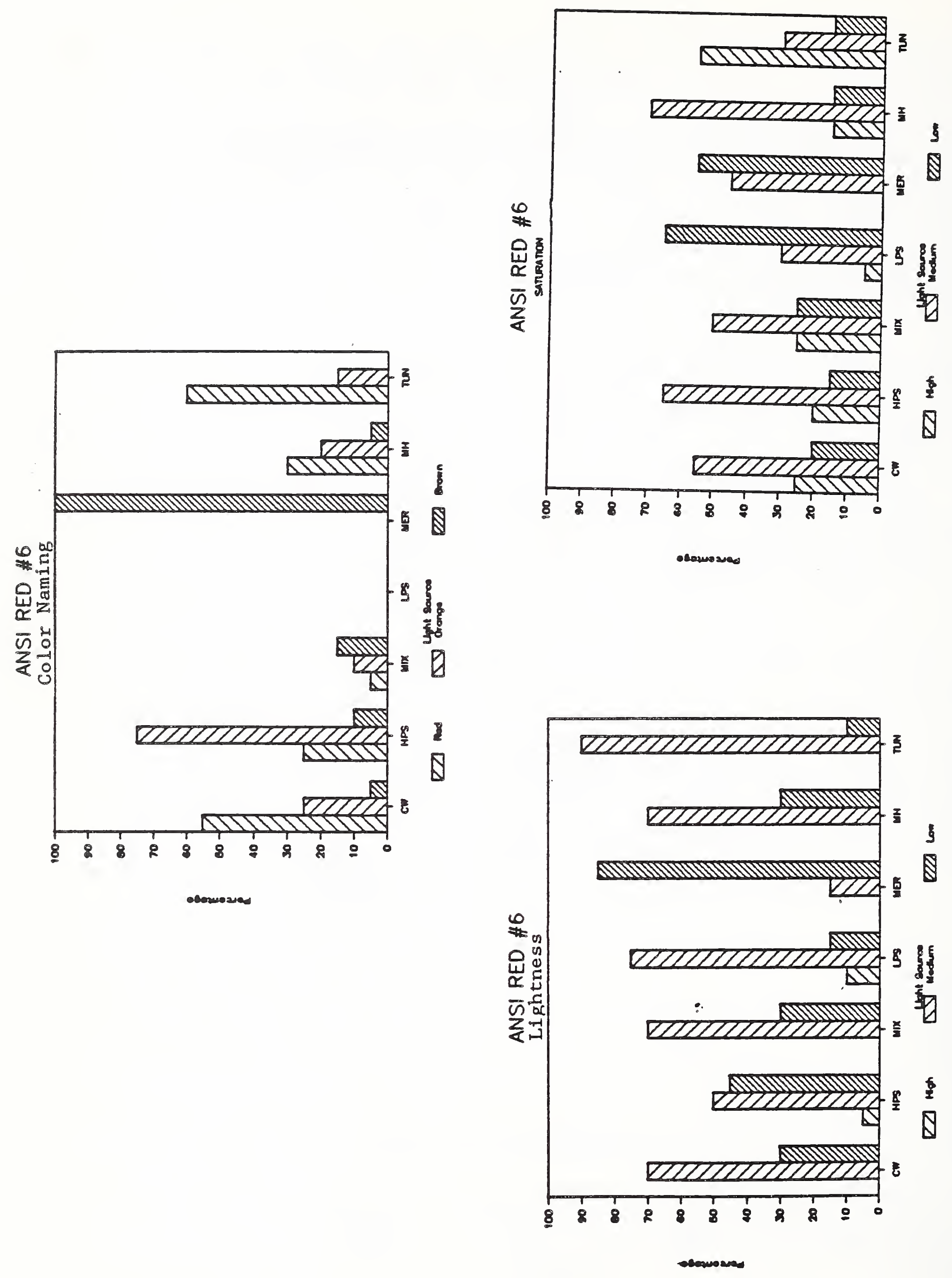

Figure 7. Color naming, lightness and saturation judgments for ANSI red \#6. 

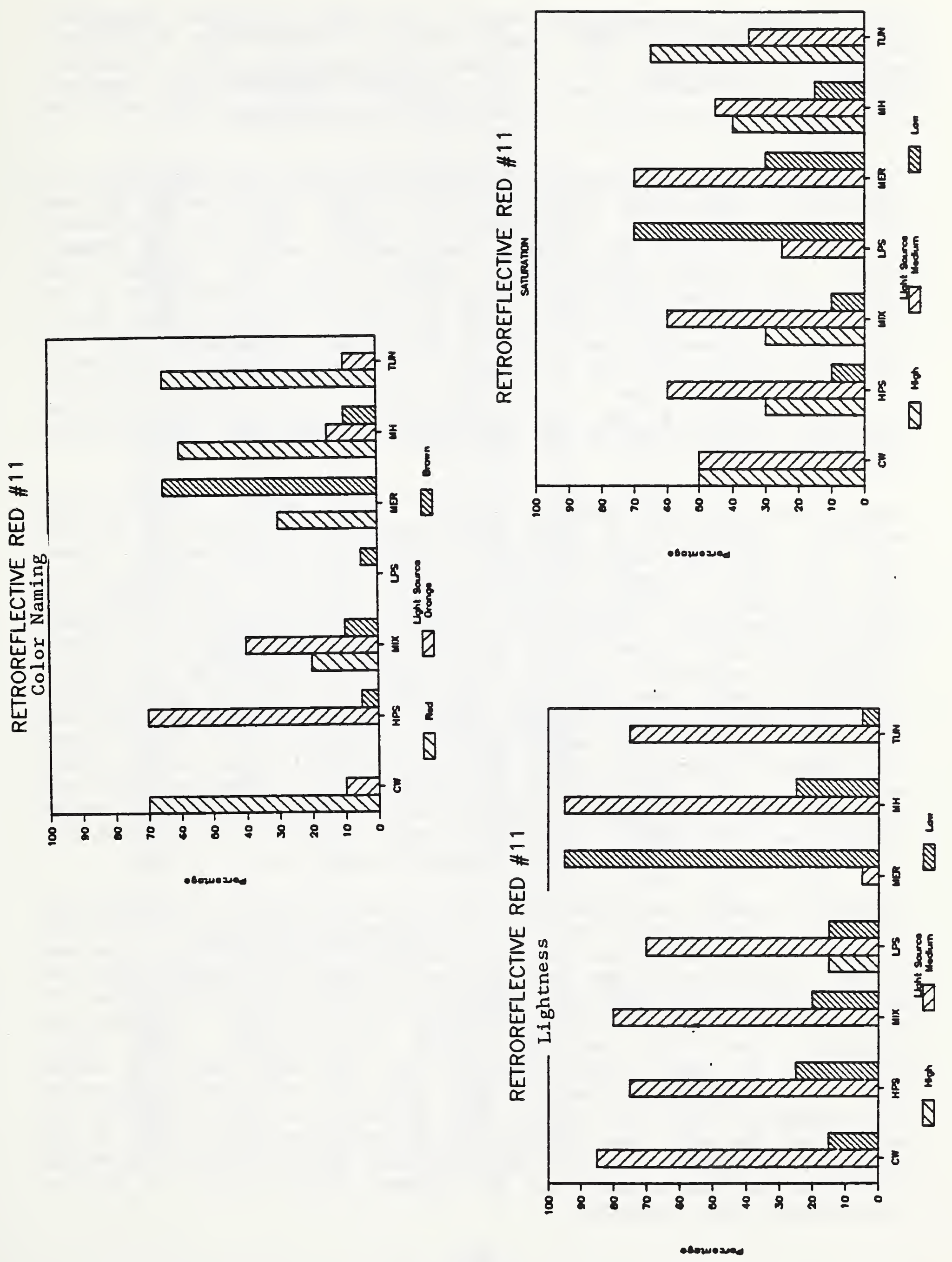

Figure 8. Color naming, lightness and saturation judgements for sample \#11 (highway red). 
Table 2 presents psychophysical data for the ANSI and highway comparison samples. The frequency that a sample was given a particular color name, primary hue percentage ( $\mathrm{PH} \%$ ), secondary hue percentage ( $\mathrm{SH} \%$ ), lightness judgement (high, medium, low), and saturation judgement (high, medium, low) is given for each source. Four primary and secondary hues were used - $\operatorname{Red}(R)$, Yellow (Y), Green (G) and Blue (B).

Inspection of table 2 reveals that highway red (\#11) is given red as a primary hue slightly more frequently for all light sources except HPS and LPS. Thus, the overall performance for highway red (\#11) is somewhat better - except under HPS and LPS. The frequent confusions with orange, however, for these light sources suggest that both samples have significant problems of accurate identification under sodium sources. A $\chi^{2}$ analysis of the differences in the frequency of color names, medium lightness judgements, and medium saturation judgements was not significant $(\mathrm{p}>.05)$ for the two samples, indicating that the differences are not particularly great. The sample that was the "best" red of those tested by Collins et al. was a fluorescent one for which extensive durability research should be done before recommending its use under outdoor conditions.

Figure 9 presents the CIELAB data for four orange samples, including ANSI and FHWA. Examination of these graphs suggests that sample \#35 is very close to the FHWA specifications, a suggestion confirmed by visual comparison with the color tolerance charts. Figures 10 and 11 present the color naming, lightness and saturation data for ANSI orange and highway orange (\#35). Examination of these figures suggests that highway orange (\#35) is a better orange than the ANSI sample since it is termed orange more frequently under all light sources. It does, however have a tendency to have lower lightness and saturation than the ANSI sample. Table 2 indicates that highway orange (\#35) is termed orange more frequently than the ANSI sample and that its primary hue is red rather than yellow. (Only four primary hues were allowed - red, yellow, green and blue.) A $\chi^{2}$ comparison of the differences in frequency counts was significant for color name, indicating that the highway orange was termed "orange" more frequently than ANSI orange $(p<.05)$. These data thus indicate that the current highway orange is effective.

Figure 12 presents the CIELAB data for six yellow samples, including ANSI and FHWA. Examination of these graphs indicates that sample \#21 is very close to the specifications both in CIELAB units and the color tolerance charts. Inspection of the lower portion of figure 12 indicates that the ANSI sample is in a different quadrant of $a * b *$ space - being closer to the $b *$ axis than the other five samples. Figures 13 and 14 present the appearance data for the ANSI sample and highway yellow (\#21). Examination of these graphs indicates that the ANSI sample is termed yellow more frequently, has a higher lightness and greater saturation than highway yellow (\#21) for all light sources. The $\chi^{2}$ comparisons were significant for color name, lightness, and saturation $(p<.05)$ for the two samples. In addition, table 2 indicates that there is a lower percentage of yellow as the primary hue for highway yellow (\#21), as well as consistently lower lightness. 
Table 2. Psychophysical Comparisons for ANSI and Highway Color Samples COLOR NAME COUNTS LIGHTNESS SATURATION ANSI RED \#6

\begin{tabular}{|c|c|c|c|c|c|c|c|c|c|c|c|}
\hline ource & Red & Orange & Brown & $\mathrm{PH} 8$ & $\mathrm{SH} \%$ & High & Med & Low & High & Med & Lo \\
\hline CW & 11 & 5 & 1 & R 85 & Y 18 & 0 & 14 & 6 & 5 & 11 & 4 \\
\hline HPS & 0 & 15 & 2 & R 69 & Y 31 & 1 & 10 & 9 & 4 & 13 & \\
\hline MIX & 2 & 9 & 3 & R 74 & Y 26 & 0 & 14 & 6 & 5 & 10 & \\
\hline LPS & 0 & 0 & 0 & Y 77 & G 24 & 2 & 15 & 3 & 1 & 6 & 13 \\
\hline MER & 0 & 0 & 20 & R 82 & Y 20 & 0 & 3 & 17 & 0 & 9 & 1 \\
\hline MH & 6 & 4 & 1 & R 79 & Y 24 & 0 & 14 & 6 & 3 & 14 & \\
\hline TUN & 12 & 3 & 0 & R 85 & Y 17 & 0 & 18 & 2 & 11 & 6 & \\
\hline
\end{tabular}

HIGHWAY RED \#11

Source Red Orange Brown $\mathrm{PH} \%$ SH\& High Med Low High Med Low

$\begin{array}{rrrrrrrrrrrrrr}\text { CW } & 14 & 2 & 0 & \mathrm{R} & 86 & \mathrm{Y} & 17 & 0 & 17 & 3 & 10 & 10 & 0 \\ \text { HPS } & 0 & 14 & 1 & \mathrm{R} & 64 & \mathrm{Y} & 36 & 0 & 15 & 5 & 6 & 12 & 2 \\ \text { MIX } & 4 & 8 & 2 & \mathrm{R} & 77 & \mathrm{Y} & 24 & 0 & 16 & 4 & 6 & 12 & 2 \\ \text { LPS } & 0 & 0 & 1 & \mathrm{Y} & 79 & \mathrm{G} & 22 & 3 & 14 & 3 & 0 & 5 & 14 \\ \text { MER } & 6 & 0 & 13 & \mathrm{R} & 87 & \mathrm{Y} & 16 & 0 & 1 & 19 & 0 & 14 & 6 \\ \text { MH } & 12 & 3 & 2 & \mathrm{R} & 82 & \mathrm{Y} & 18 & 0 & 15 & 5 & 8 & 9 & 3 \\ \text { TUN } & 13 & 2 & 0 & \mathrm{R} & 87 & \mathrm{Y} & 16 & 0 & 19 & 1 & 13 & 7 & 0\end{array}$

ANSI ORANGE \#5

\begin{tabular}{|c|c|c|c|c|c|c|c|c|c|c|}
\hline Source & Orange & Yellow & $\mathrm{PH}$ 웅 & $\mathrm{SH} \%$ & High & Med & Low & High & Med & Low \\
\hline CW & 13 & 0 & Y 72 & R 28 & 2 & 18 & 0 & 1 & 19 & 0 \\
\hline HPS & 5 & 13 & Y 88 & R 16 & 6 & 13 & 1 & 10 & 10 & 0 \\
\hline MIX & 10 & 2 & Y 82 & R 21 & 1 & 19 & 0 & 5 & 13 & 2 \\
\hline LPS & 0 & 20 & Y 96 & R 06 & 9 & 11 & 0 & 10 & 6 & 4 \\
\hline MER & 0 & 12 & Y 93 & R 13 & 1 & 17 & 2 & 1 & 12 & 7 \\
\hline MH & 11 & 1 & Y 74 & R 28 & 2 & 17 & 1 & 3 & 13 & 4 \\
\hline TUN & 17 & 1 & Y 76 & R 24 & 3 & 16 & 1 & 3 & 15 & 2 \\
\hline
\end{tabular}

HIGHWAY ORANGE \#35

\begin{tabular}{|c|c|c|c|c|c|c|c|c|c|c|c|}
\hline Source & Orange & Yellow & Olive & $\mathrm{PH}$ 영 & $\mathrm{SH} \%$ & High & Med & Low & High & Med & Low \\
\hline CW & 13 & 1 & 0 & Y 70 & R 31 & 2 & 16 & 2 & 0 & 16 & 4 \\
\hline HPS & 16 & 0 & 0 & Y 69 & R 31 & 3 & 17 & 0 & 4 & 15 & 1 \\
\hline MIX & 12 & 1 & 0 & Y 78 & R 22 & 1 & 16 & 3 & 3 & 13 & \\
\hline LPS & 0 & 19 & 1 & Y 96 & G 11 & 7 & 12 & 1 & 7 & 7 & \\
\hline MER & 1 & 6 & 7 & Y 84 & G 18 & 0 & 15 & 5 & 0 & 7 & 13 \\
\hline MH & 16 & 1 & 0 & Y 78 & R 24 & 1 & 16 & 3 & 1 & 14 & \\
\hline TUN & 19 & 0 & 0 & R 61 & Y 39 & 1 & 17 & 2 & 4 & 12 & \\
\hline
\end{tabular}


ANSI YELLOW \#4

\begin{tabular}{ccr} 
Source & Yellow & \multicolumn{1}{c}{ YG } \\
CW & 15 & 4 \\
HPS & 19 & 1 \\
MIX & 17 & 2 \\
LPS & 20 & 0 \\
MER & 8 & 10 \\
MH & 16 & 4 \\
TUN & 17 & 2
\end{tabular}

PHo 5 SH 8 High Med Low High Med Low

$\begin{array}{llllllllll}Y & 90 & G & 13 & 12 & 8 & 0 & 6 & 14 & 0\end{array}$

$\begin{array}{llllllllll}Y & 93 & G & 12 & 14 & 6 & 0 & 11 & 7 & 2\end{array}$

$\begin{array}{llllllllll}Y & 94 & G & 12 & 12 & 8 & 0 & 6 & 14 & 0\end{array}$

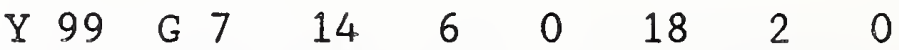

$\begin{array}{llllllllll}Y & 85 & G & 18 & 11 & 9 & 0 & 10 & 9 & 1\end{array}$

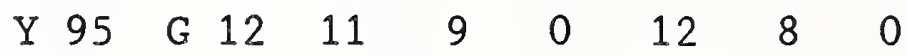

HIGHWAY YELLOW \#21

Source Yellow Gold Orange $\mathrm{PH} \%$ SHo High Med Low High Med Low

$\begin{array}{rrllllllllllll}\text { CW } & 11 & 3 & 3 & \text { Y } & 94 & \text { R } & 8 & 4 & 16 & 0 & 3 & 14 & 3 \\ \text { HPS } & 18 & 0 & 1 & \text { Y } & 95 & \text { G } & 12 & 7 & 13 & 0 & 8 & 10 & 2 \\ \text { MIX } & 10 & 2 & 5 & \text { Y } & 88 & \text { R } & 17 & 5 & 15 & 0 & 6 & 13 & 1 \\ \text { LPS } & 19 & 1 & 0 & \text { Y } & 97 & \text { G } & 9 & 8 & 12 & 0 & 9 & 9 & 2 \\ \text { MER } & 13 & 3 & 0 & \text { Y } & 96 & \text { G } & 8 & 1 & 19 & 0 & 2 & 16 & 2 \\ \text { MH } & 5 & 6 & 7 & \text { Y } & 85 & \text { R } & 22 & 4 & 14 & 2 & 2 & 14 & 4 \\ \text { TUN } & 6 & 6 & 6 & \text { Y } & 85 & \text { R } & 20 & 5 & 14 & 1 & 3 & 13 & 4\end{array}$

ANSI GREEN \#3

\begin{tabular}{|c|c|c|c|c|c|c|c|c|c|c|c|}
\hline Source & Green & $B G$ & Gray & PHO & SH용 & High & Med & Low & High & Med & Low \\
\hline CW & 11 & 9 & 0 & G 84 & B 20 & 0 & 12 & 8 & 1 & 16 & 3 \\
\hline HPS & 8 & 10 & 0 & G 81 & B 26 & 0 & 7 & 13 & 1 & 13 & 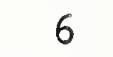 \\
\hline MIX & 10 & 9 & 0 & G 84 & B 22 & 0 & 13 & 7 & 0 & 17 & 3 \\
\hline LPS & 1 & 0 & 13 & $\mathrm{G} / \mathrm{R}$ & Y 18 & 0 & 2 & 18 & 0 & 3 & 1 \\
\hline MER & 17 & 2 & 0 & G 89 & B 19 & 0 & 10 & 10 & 0 & 7 & 13 \\
\hline MH & 12 & 8 & 0 & G 87 & B 17 & 0 & 16 & 4 & 1 & 15 & 4 \\
\hline TUN & 13 & 7 & 0 & G 58 & B 14 & 0 & 17 & 3 & 4 & 14 & 0 \\
\hline
\end{tabular}

HIGHWAY GREEN \#25

\begin{tabular}{rrrrllllllllll} 
Source & Green & BG & Black & PH8 & \multicolumn{3}{l}{ SH8 } & High & Med & Low & High & Med Low \\
CW & 11 & 8 & 0 & G & 85 & B & 20 & 1 & 13 & 6 & 1 & 17 & 2 \\
HPS & 1 & 17 & 0 & B & 67 & G & 33 & 0 & 5 & 15 & 4 & 12 & 4 \\
MIX & 4 & 15 & 0 & G & 77 & B & 27 & 0 & 13 & 7 & 5 & 14 & 1 \\
LPS & 0 & 0 & 14 & & 0 & & 0 & 0 & 0 & 20 & 0 & 0 & 1 \\
MER & 17 & 1 & 0 & G & 92 & B & 12 & 0 & 9 & 11 & 1 & 13 & 6 \\
MH & 8 & 12 & 0 & G & 79 & B & 28 & 1 & 15 & 4 & 3 & 16 & 1 \\
TUN & 7 & 13 & 0 & G & 74 & B & 28 & 0 & 16 & 4 & 8 & 12 & 0
\end{tabular}


ANSI BLUE \#2

\begin{tabular}{|c|c|c|c|c|c|c|c|c|c|c|c|}
\hline Source & Blue & Purple & Gray & $\mathrm{PH}$ 웅 & $\mathrm{SH}$ 웅 & High & Med & Low & High & Med & Lo \\
\hline $\mathrm{CW}$ & 20 & 0 & 0 & В 99 & $\mathrm{R}$ & 0 & 14 & 6 & 6 & 12 & 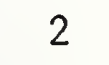 \\
\hline HPS & 20 & 0 & 0 & B 98 & $R / G$ & 0 & 7 & 13 & 4 & 12 & \\
\hline MIX & 20 & 0 & 0 & В 99 & $\mathrm{R}$ & 0 & 13 & 7 & 5 & 13 & \\
\hline LPS & 0 & 0 & 12 & R 80 & Y 16 & 0 & 0 & 20 & 0 & 0 & \\
\hline MER & 12 & 8 & 0 & B 85 & $\begin{array}{lll}R & 19\end{array}$ & 8 & 10 & 10 & 3 & 12 & \\
\hline $\mathrm{MH}$ & 20 & 0 & 0 & B 98 & $\mathrm{R}$ & 0 & 17 & 3 & 4 & 12 & \\
\hline TUN & 20 & 0 & 0 & B 97 & G & 0 & 15 & 5 & 5 & 13 & \\
\hline
\end{tabular}

HIGHWAY BLUE \#27

\begin{tabular}{|c|c|c|c|c|c|c|c|c|c|c|}
\hline Source & Blue & Black & $\mathrm{PH} \%$ & $\mathrm{SH}$ 웅 & High & Med & Low & High & Med & Lo \\
\hline$C W$ & 20 & 0 & B 99 & R 5 & 0 & 11 & 9 & 12 & 8 & 0 \\
\hline HPS & 20 & 0 & B 98 & $\mathrm{R} / \mathrm{G}$ & 0 & 3 & 17 & 12 & 8 & \\
\hline MIX & 20 & 0 & B 99 & $R / G$ & 0 & 13 & 7 & 12 & 7 & \\
\hline LPS & 5 & 15 & B 100 & 0 & 0 & 0 & 20 & 1 & 2 & \\
\hline MER & 19 & 0 & B 96 & $\mathrm{R}$ & 0 & 1 & 19 & 8 & 12 & \\
\hline $\mathrm{MH}$ & 20 & 0 & B 98 & $R / G$ & 0 & 15 & 5 & 11 & 9 & \\
\hline TUN & 20 & 0 & B 98 & $\mathrm{R} / \mathrm{G}$ & 1 & 13 & 6 & 12 & 8 & \\
\hline
\end{tabular}

ANSI BROWN \#7

Source Brown Tan Olive $\mathrm{PH} \%$ SH8 High Med Low High Med Low

$\begin{array}{rrlllllllrrrrr}\text { CW } & 20 & 0 & 0 & \mathrm{R} & 75 & \mathrm{Y} & 24 & 0 & 3 & 17 & 0 & 13 & 7 \\ \text { HPS } & 11 & 1 & 7 & \mathrm{R} & 79 & \mathrm{Y} & 25 & 0 & 7 & 13 & 0 & 10 & 10 \\ \text { MIX } & 15 & 3 & 2 & \mathrm{R} & 79 & \mathrm{Y} & 21 & 0 & 5 & 15 & 1 & 10 & 9 \\ \text { LPS } & 2 & 6 & 6 & \mathrm{G} & 87 & \mathrm{Y} & 14 & 3 & 11 & 6 & 0 & 5 & 13 \\ \text { MER } & 11 & 1 & 7 & \mathrm{G} & 86 & \mathrm{Y} & 10 & 0 & 7 & 13 & 0 & 6 & 14 \\ \text { MH } & 18 & 2 & 0 & \mathrm{R} & 76 & \mathrm{Y} & 26 & 0 & 4 & 16 & 1 & 9 & 10 \\ \text { TUN } & 19 & 0 & 0 & \mathrm{R} & 79 & \mathrm{Y} & 20 & 0 & 3 & 17 & 0 & 9 & 11\end{array}$

HIGHWAY BROWN \#38

Source Brown Tan Olive PH\% SHo High Med Low High Med Low

\begin{tabular}{|c|c|c|c|c|c|c|c|c|c|c|c|}
\hline CW & 20 & 0 & 0 & R 79 & $\mathrm{R} / \mathrm{Y}$ & 0 & 4 & 16 & 1 & 11 & 8 \\
\hline HPS & 13 & 1 & 6 & R 80 & Y 23 & 1 & 7 & 12 & 0 & 10 & 10 \\
\hline MIX & 18 & 0 & 2 & R 79 & R 34 & 1 & 5 & 14 & 0 & 10 & 10 \\
\hline LPS & 2 & 4 & 8 & G 88 & Y 17 & 1 & 12 & 7 & 0 & 3 & 14 \\
\hline MER & 14 & 0 & 5 & G 84 & Y 9 & 0 & 3 & 17 & 1 & 8 & 11 \\
\hline $\mathrm{MH}$ & 16 & 1 & 1 & R 78 & Y 20 & 0 & 4 & 16 & 1 & 12 & 7 \\
\hline TUN & 20 & 0 & 0 & R 78 & Y 21 & 0 & 6 & 14 & 1 & 9 & 10 \\
\hline
\end{tabular}


ANSI WHITE \#10

$\begin{array}{crc}\text { Source } & \text { White } & \text { Yellow } \\ \text { CW } & 20 & 0 \\ \text { HPS } & 20 & 0 \\ \text { MIX } & 20 & 0 \\ \text { LPS } & 0 & 20 \\ \text { MER } & 19 & 0 \\ \text { MH } & 19 & 0 \\ \text { TUN } & 20 & 0\end{array}$

PH\& SHo High Med Low High Med Low

$\begin{array}{llllll}20 & 0 & 0 & 0 & 0 & 0 \\ 20 & 0 & 0 & 0 & 0 & 0\end{array}$

$\begin{array}{llllll}20 & 0 & 0 & 0 & 0 & 0\end{array}$

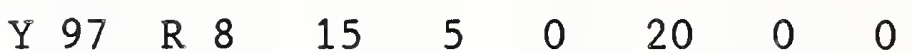

$\begin{array}{llllll}20 & 0 & 0 & 0 & 0 & 0\end{array}$

$\begin{array}{llllll}20 & 0 & 0 & 0 & 0 & 0\end{array}$

$\begin{array}{llllll}20 & 0 & 0 & 0 & 0 & 0\end{array}$

RETROREFLECTIVE WHITE \#31

\begin{tabular}{|c|c|c|c|c|c|c|c|c|c|c|c|}
\hline Source & White & Yellow & Gray & $\mathrm{PH} \%$ & $\mathrm{SH} 8$ & High & Med & Low & High & Med & Low \\
\hline CW & 8 & 0 & 12 & & & 18 & 2 & 0 & 1 & 0 & 2 \\
\hline HPS & 5 & 0 & 13 & & & 15 & 5 & 0 & 0 & 1 & \\
\hline MIX & 4 & 0 & 15 & & & 16 & 4 & 0 & 0 & 0 & \\
\hline LPS & 0 & 13 & 0 & Y 94 & G 9 & 5 & 15 & 0 & 6 & 9 & \\
\hline MER & 5 & 0 & 15 & & & 15 & 5 & 0 & 0 & 0 & \\
\hline MH & 3 & 0 & 17 & & & 15 & 5 & 0 & 0 & 0 & \\
\hline TUN & 4 & 0 & 16 & & & 15 & 5 & 0 & 0 & 0 & \\
\hline
\end{tabular}



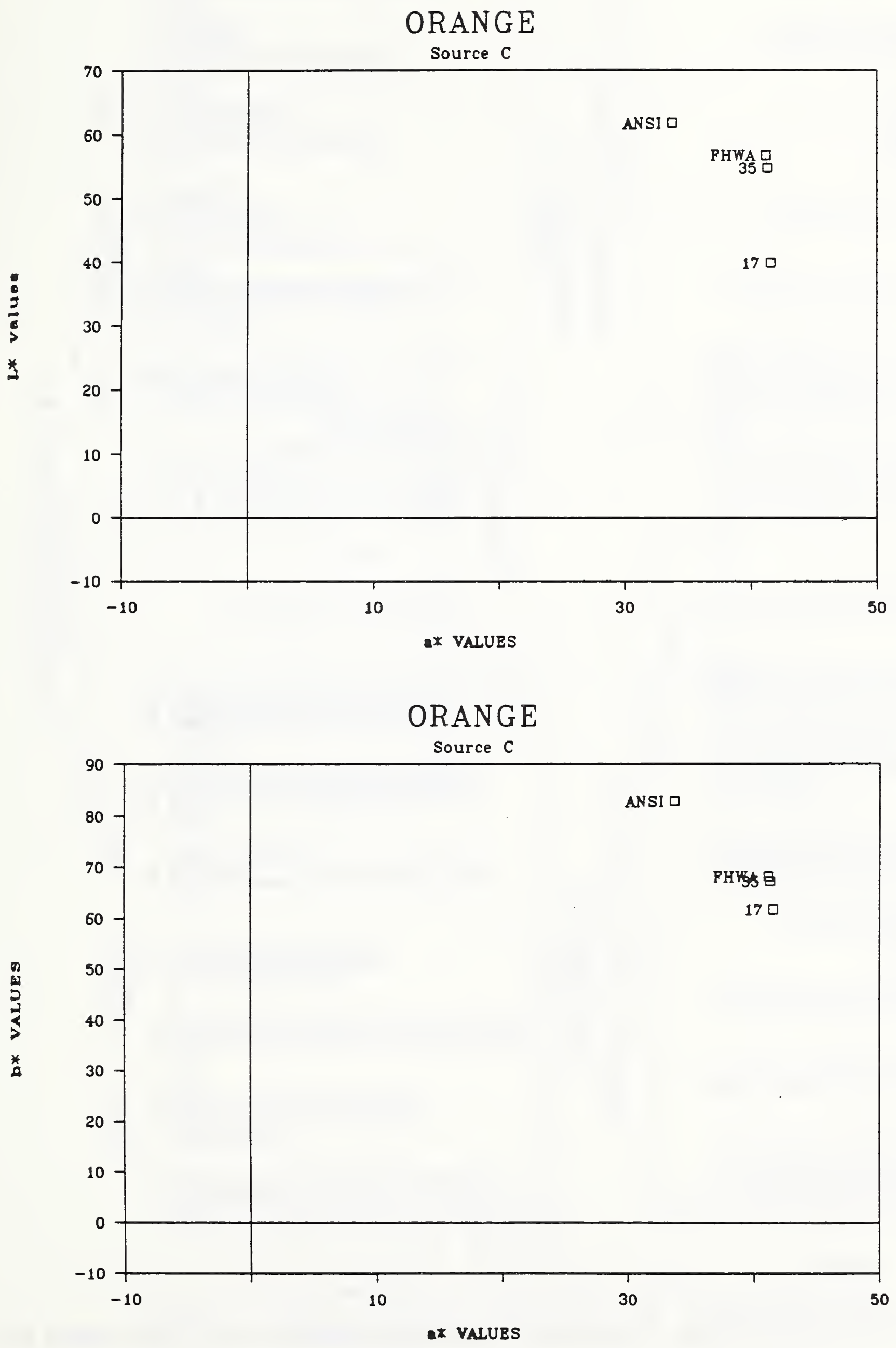

Figure 9. CIELAB comparison data for orange samples. 

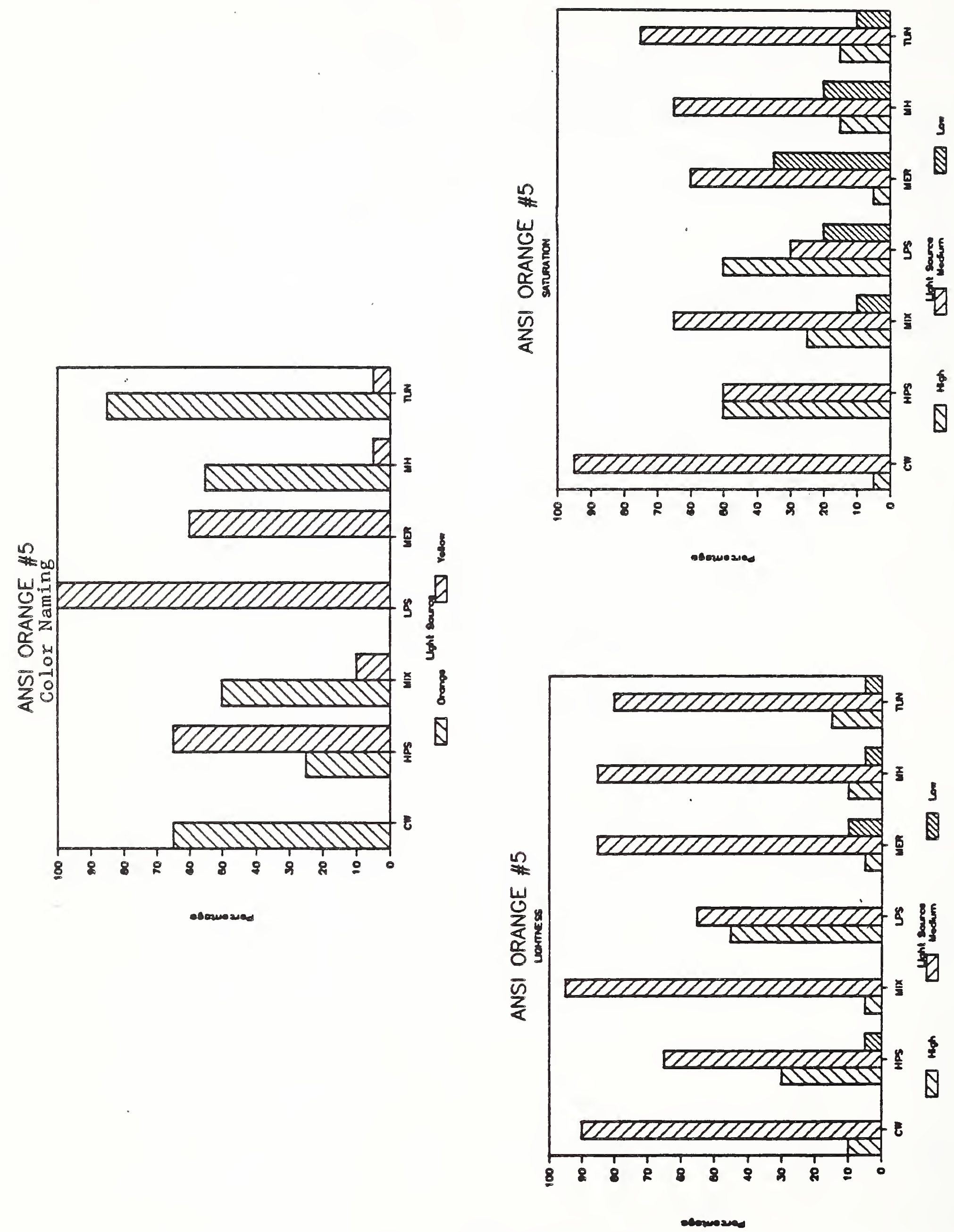

Figure 10. Color naming, lightness, and saturation data for ANSI orange \#5. 

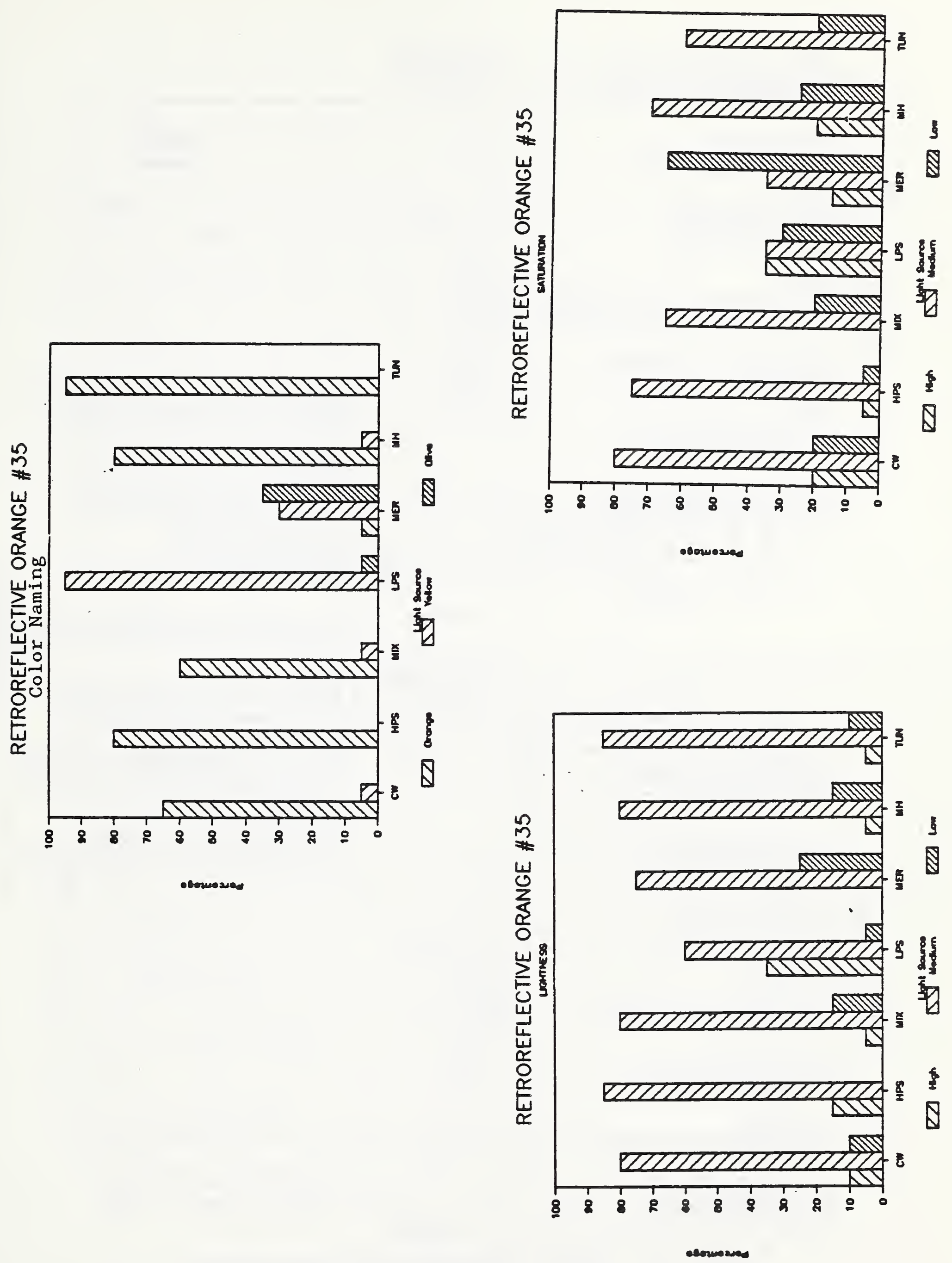

Figure 11 Color naming, lightness, and saturation data for sample \#35 (highway orange). 

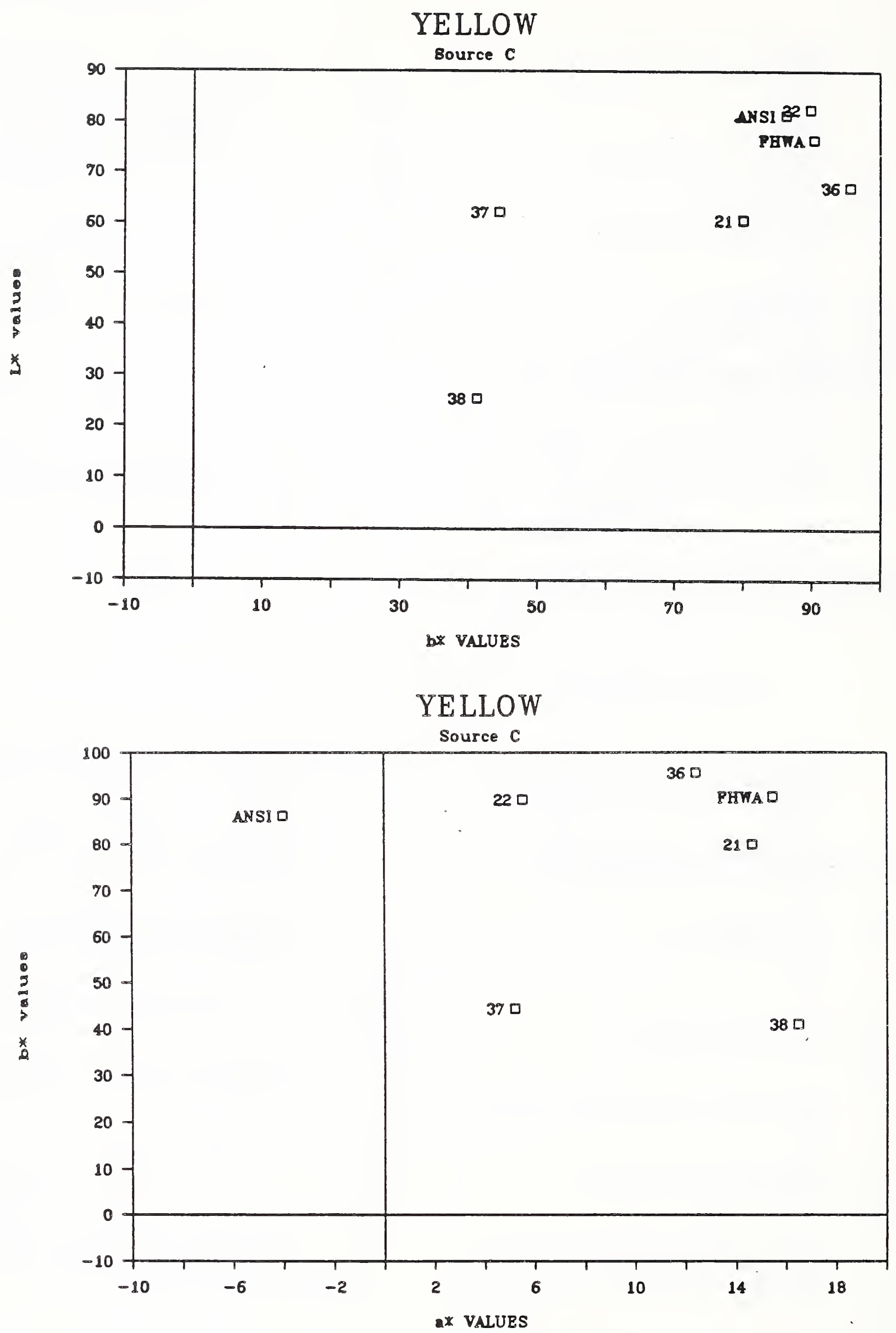

Figure 12. CIELAB comparisons for yellow samples. 

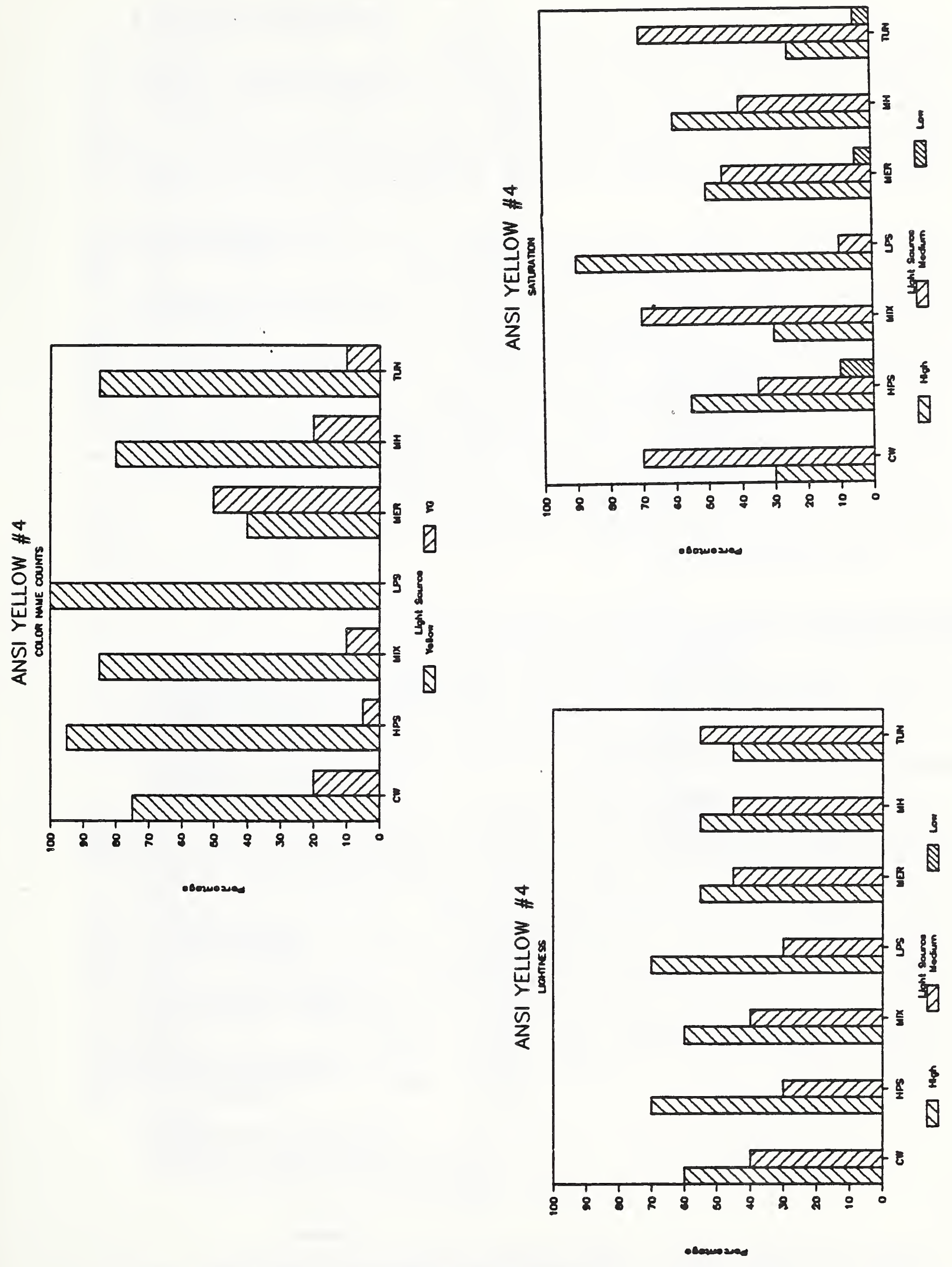

Figure 13 Color naming, lightness, and saturation data for ANSI yellow. 

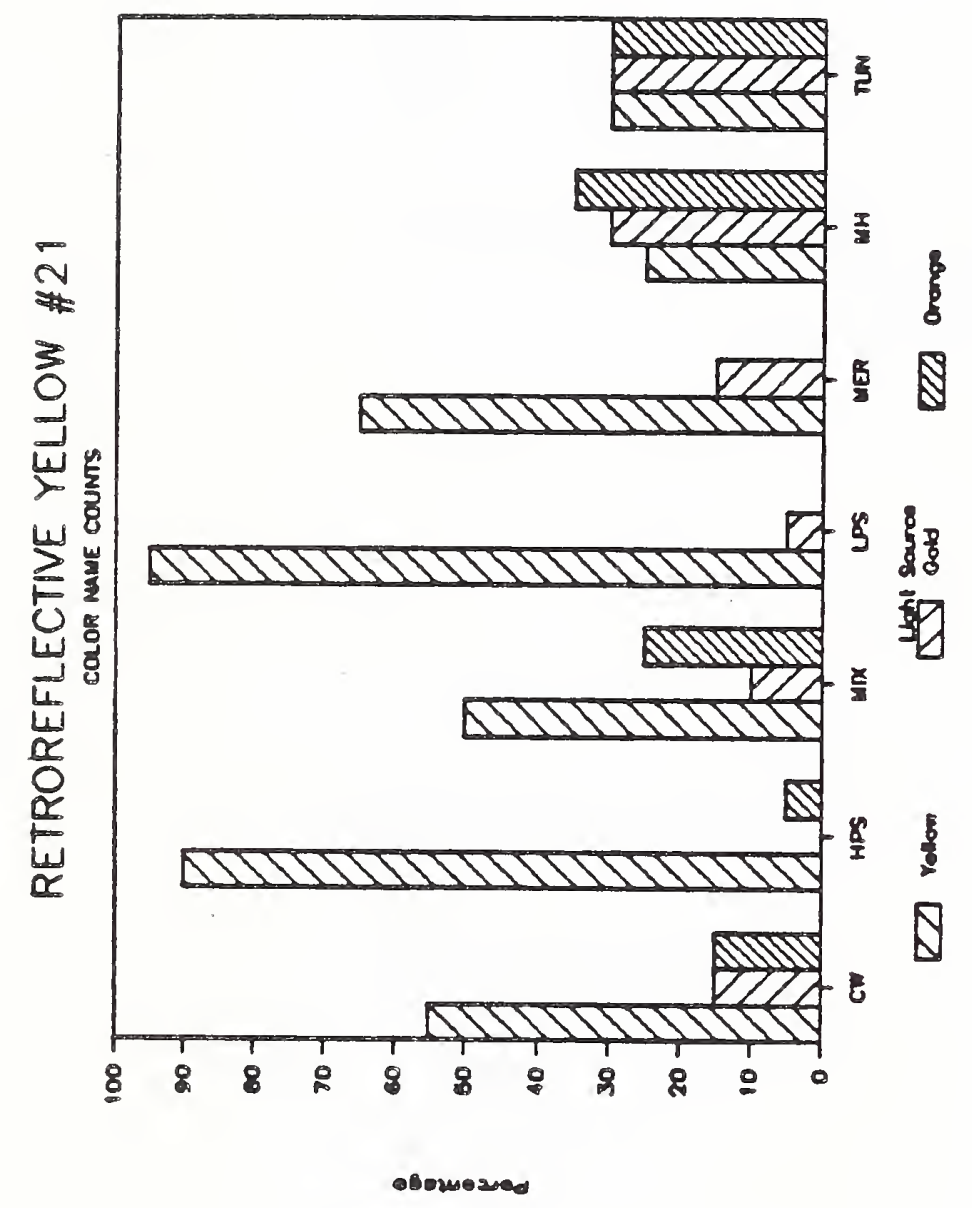
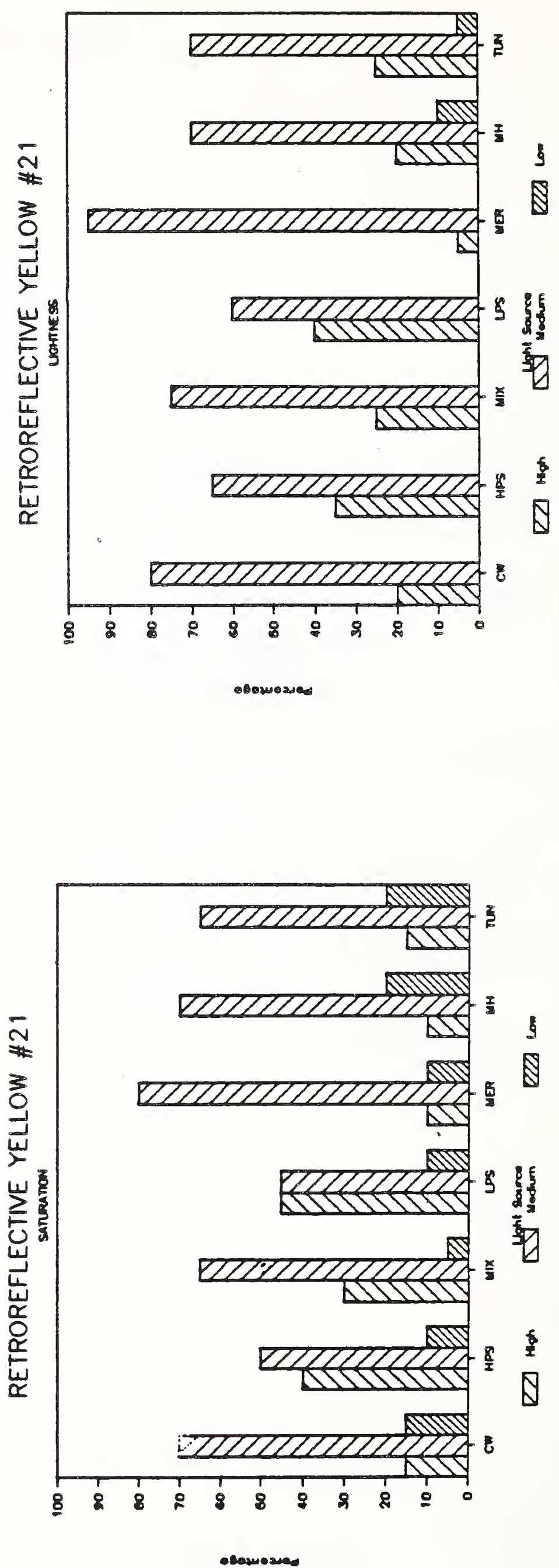

Figure 14 Color naming, lightness, and saturation data for sample \#21
(highway yellow) 
Examination of these data indicates that this sample is also termed yellow less frequently than the ANSI yellow, and has lower lightness and saturation. These comparisons suggest that samples such as \#21 and \#36 are not as good a yellow as the ANSI yellow, because they have a large number of confusions with orange, and are seen as darker, less saturated colors. Darker colors will also contrast somewhat less well with black than lighter yellow, although such contrast differences are far too small to be of practical significance.

Figure 15 presents the CIELAB data for four green samples, including ANSI and FHWA. These graphs suggest that highway green (\#25) is closest to the specifications for green but that the ANSI sample has higher lightness as measured by $L^{*}$. Figures 16 and 17 present color appearance data for highway green (\#25) and the ANSI sample which suggest that the ANSI color is seen as green more frequently than highway green (\#25). The latter is seen as "blue-green" under HPS, MIX, MH and TUN, whereas the ANSI sample is seen as "green". Both samples have generally medium saturation and medium to low lightness. The $\chi^{2}$ comparison for color name was significant $(p<.05)$. Table 2 indicates that highway green (\#25) tends to have lower percentages of green as the primary hue - and in fact is given blue as the primary hue for HPS. These data suggest that the ANSI green is a better green than highway green (\#25). Both samples are bluer greens rather than yellower greens to minimize confusions for color defective observers.

Finally, figure 18 presents CIELAB data for blue samples which suggest that sample 27 is closest to the FHWA specifications, although the agreement is poorest for the blue samples. Figures 19 and 20 present color appearance data for ANSI blue and sample \#27. These data indicate that the ANSI blue is termed blue slightly more frequently than sample 27 (except under mercury light) and is seen as being lighter. It has a significantly lower saturation, however, according to the $\chi^{2}$ analysis.

An analysis in which the data for ANSI brown and highway brown were compared was not significant. The two samples are very close in CIELAB space so that there should have been no difference in psychophysical results, as indicated by Table 2. This table does indicate problems for the white retroreflective sample which tends to be termed gray under diffuse viewing conditions. High intensity white retroreflective samples have an even greater tendency to be termed gray, except when viewed under directional illumination. (It should be noted that these white samples may represent the effective light limit for this type of retroreflective sheeting.) This may not be a problem since retroreflective white is typically used as contrast color for legends against a colored background. Care should be taken, though, to maintain good contrast between background and legend. 


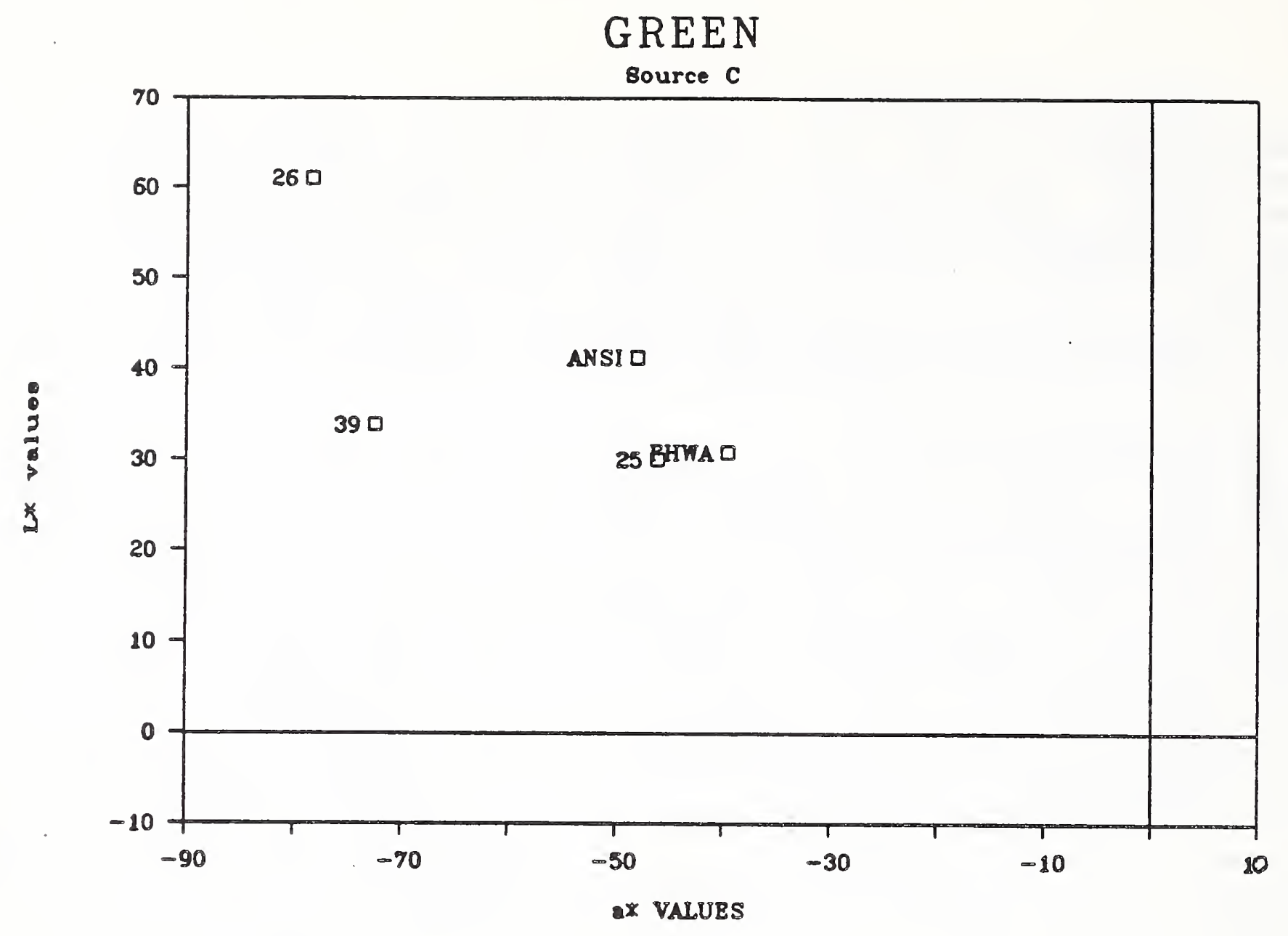

GREEN

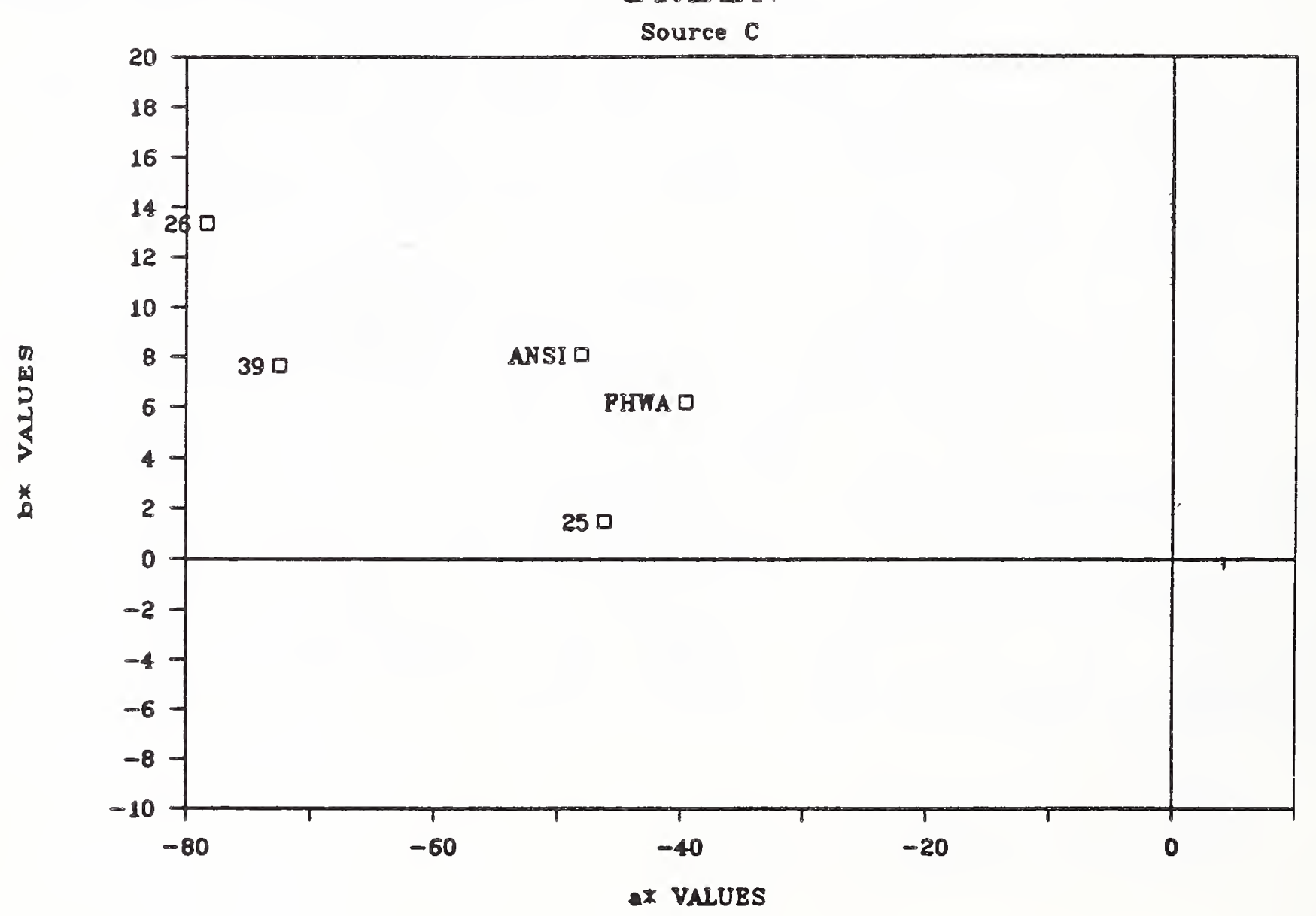

Figure 15. CIELAB comparisons for green samples. 


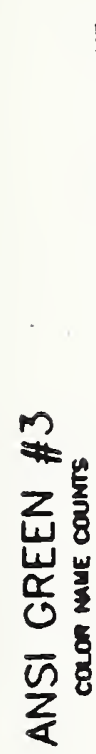

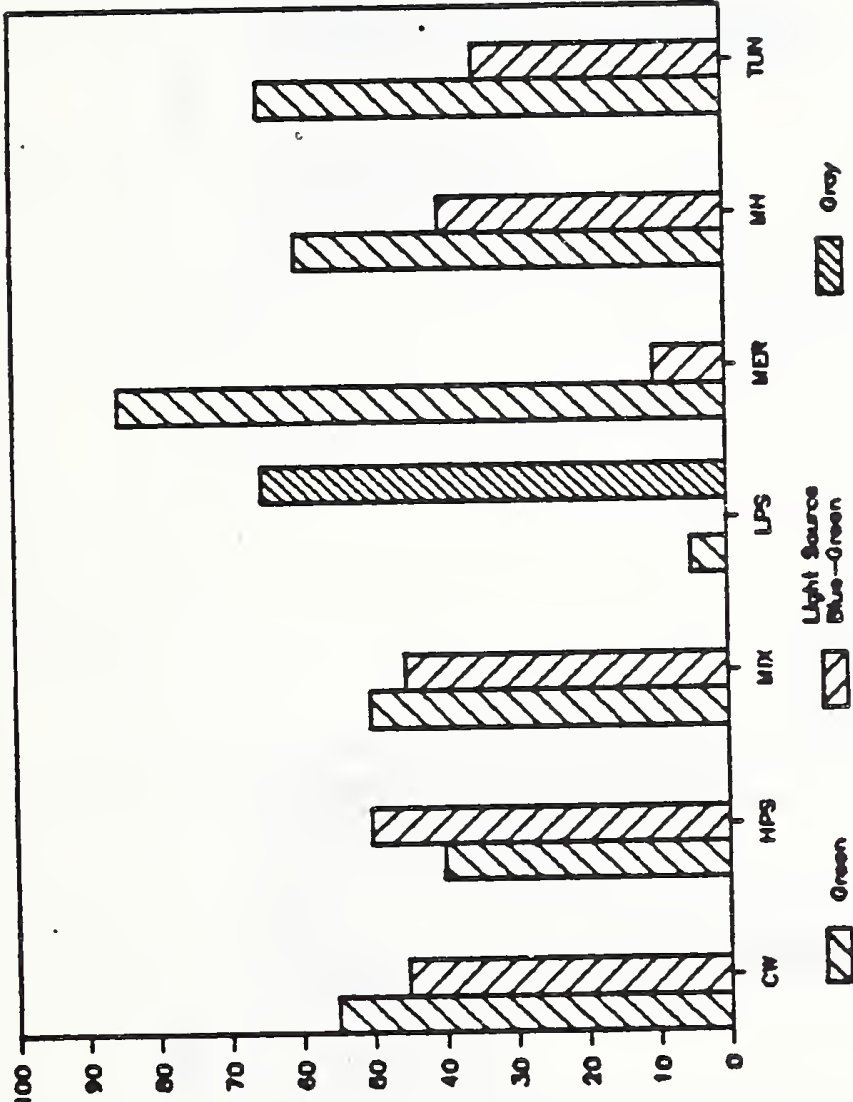

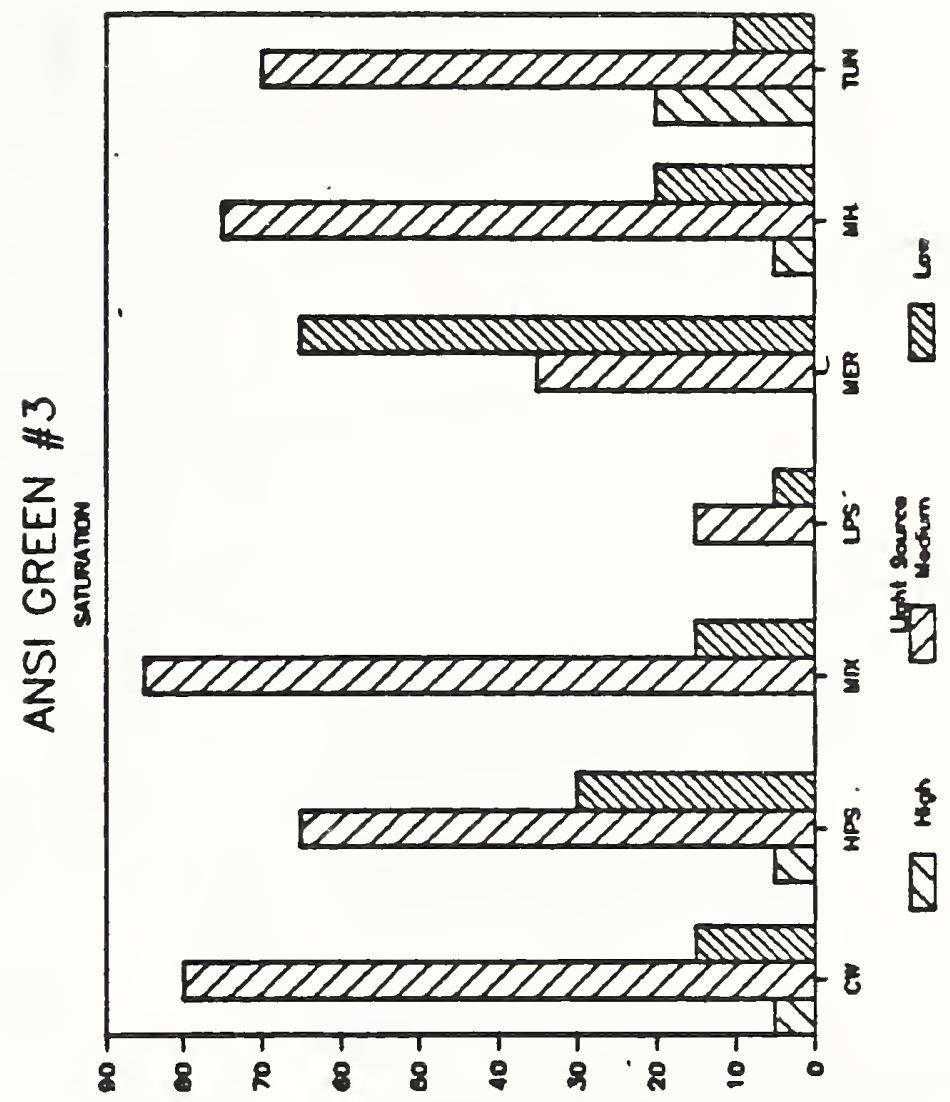

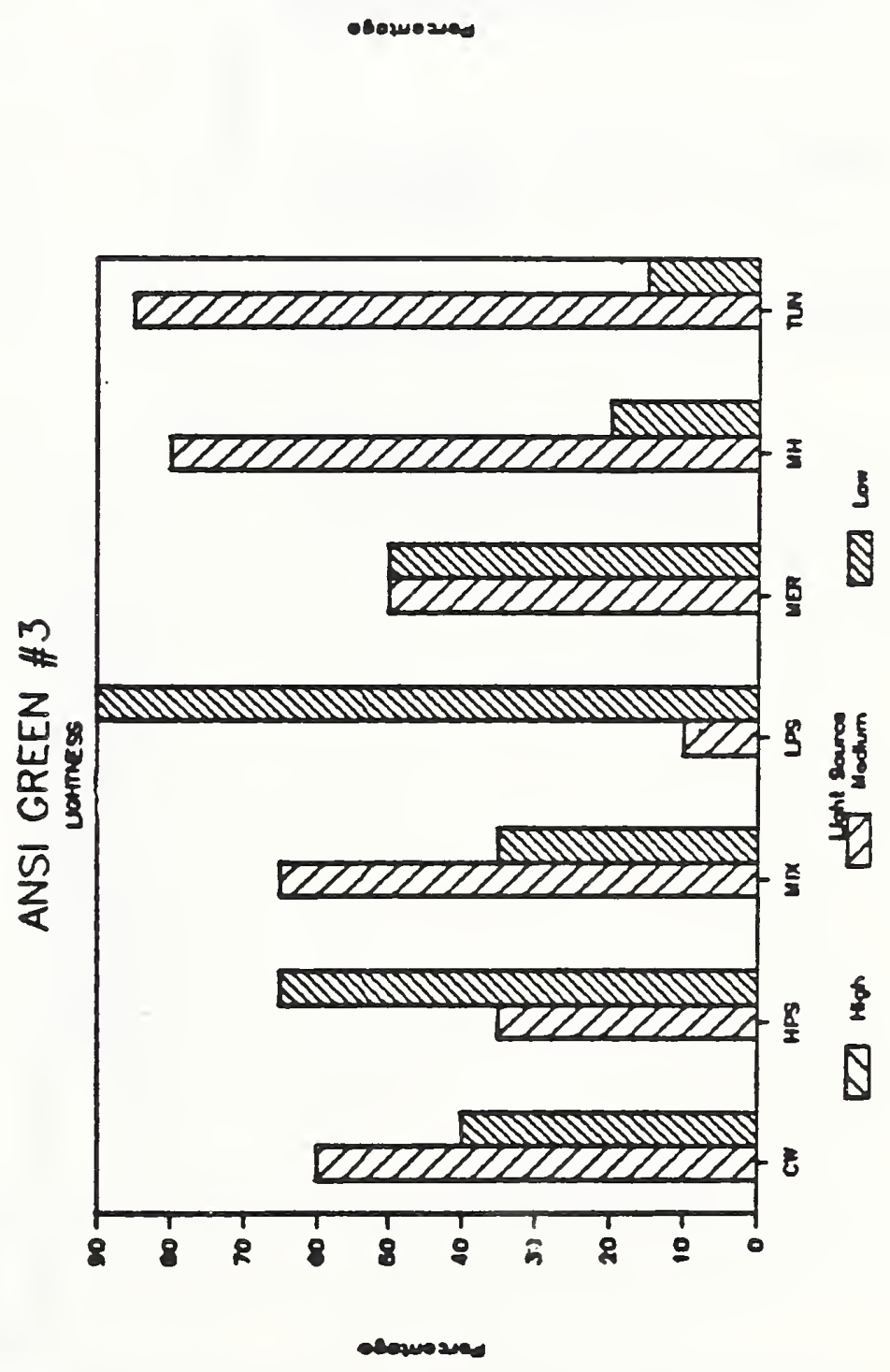

Figure 16 Color naming, lightness, and saturation data for ANSI green. 


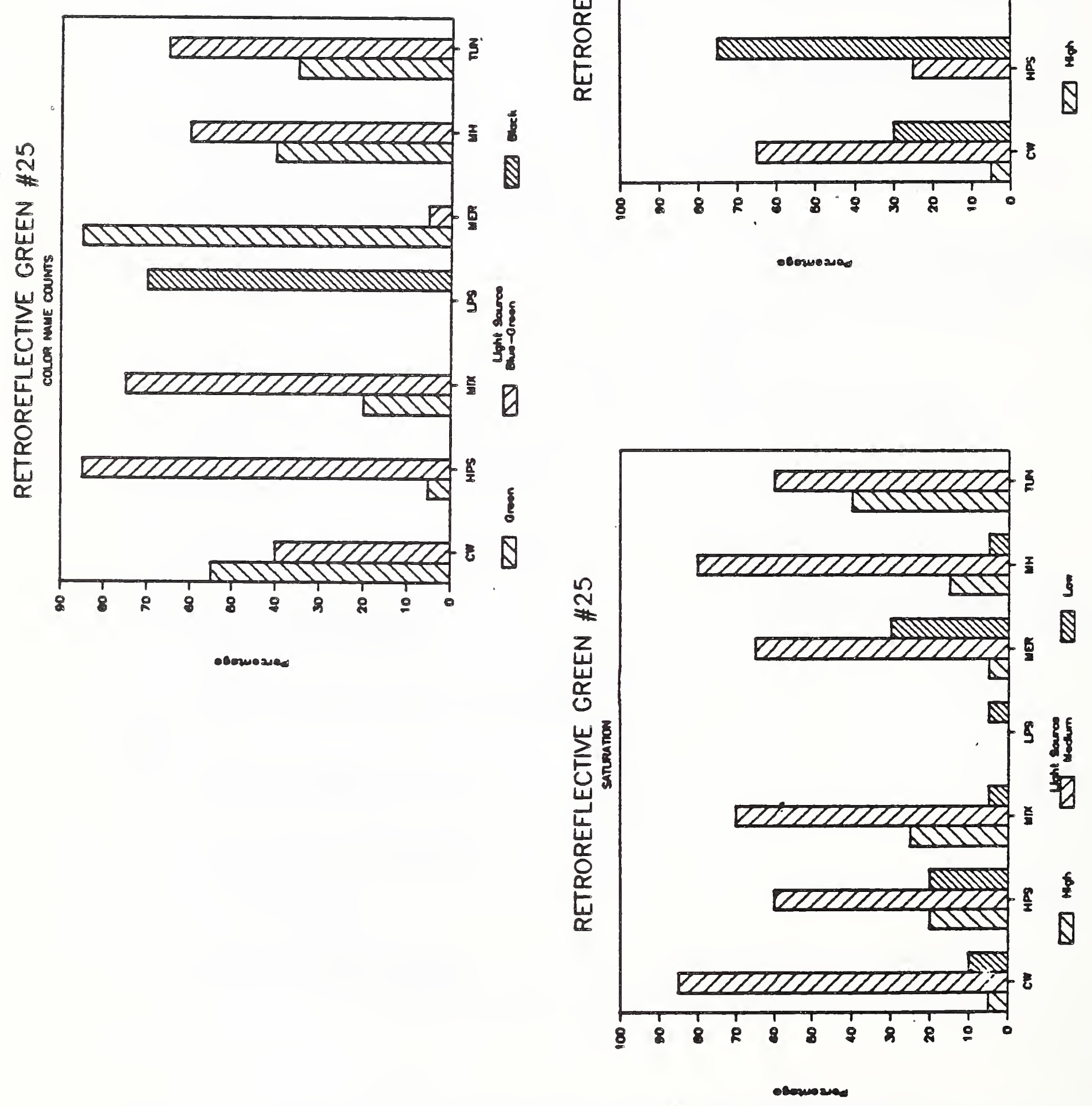

Figure 17 Color naming, lightness, and saturation data for sample \#25 (highway green). 


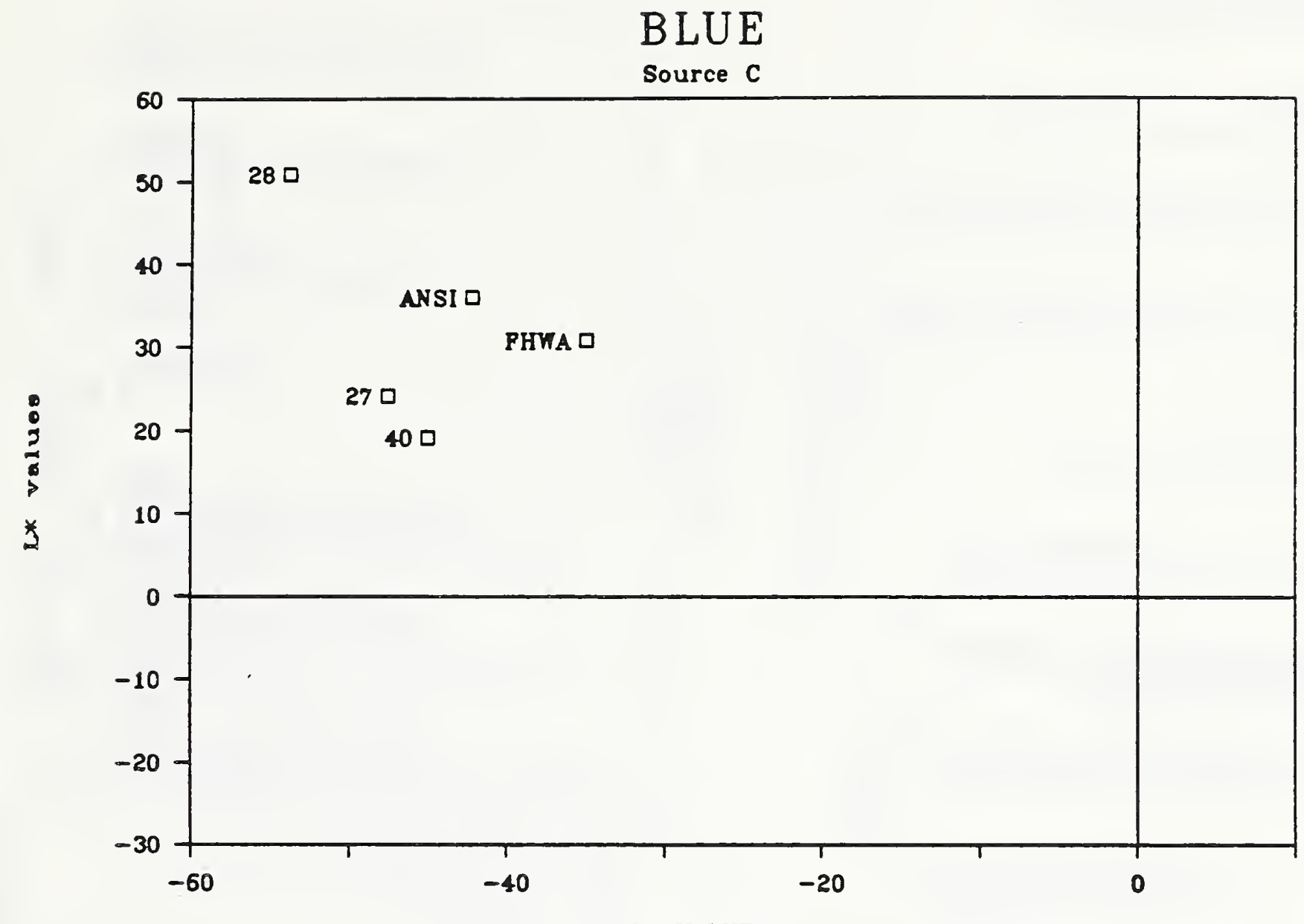

b* VALUES

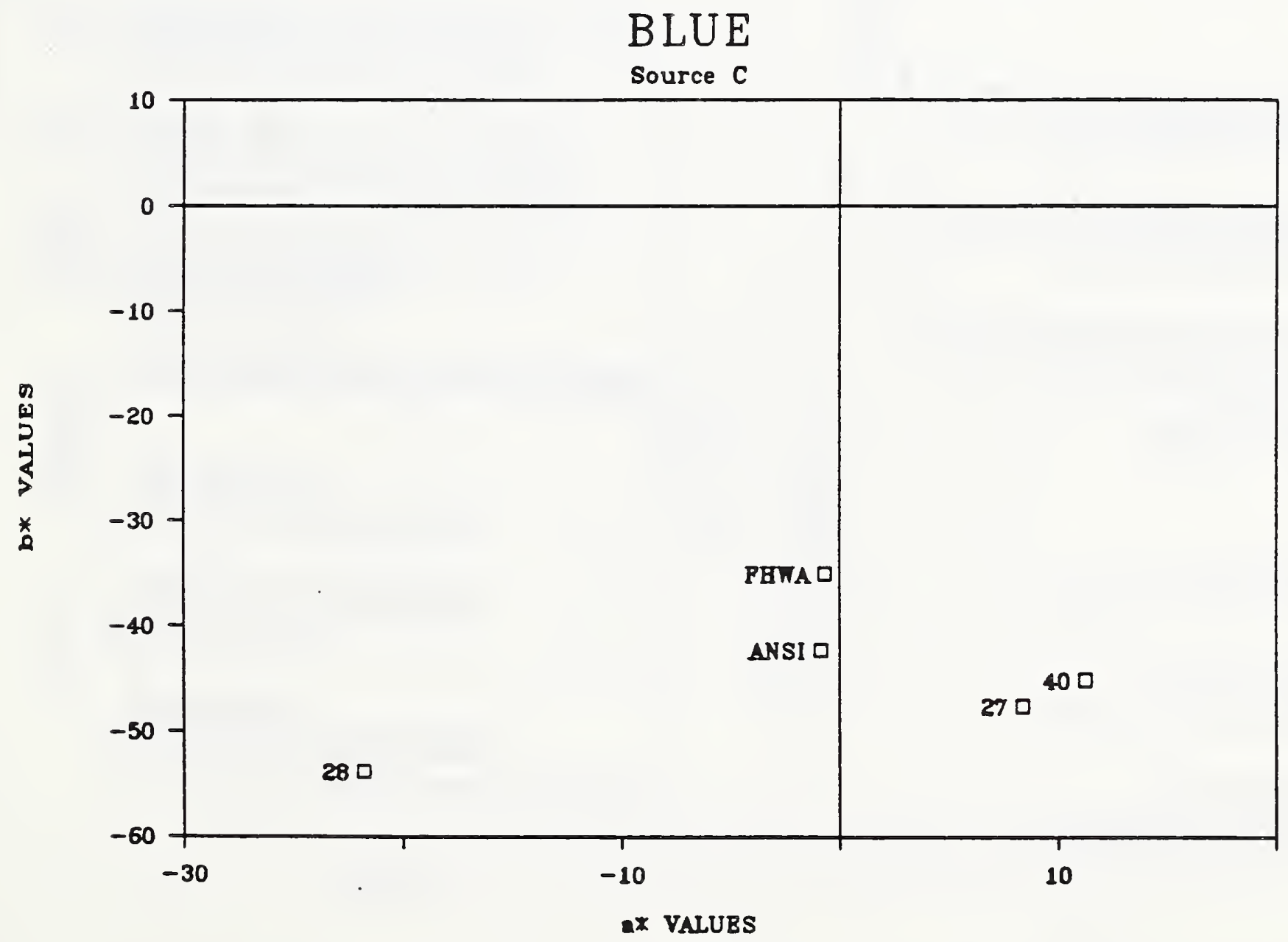

Figure 18. CIELAB comparisons for blue samples. 

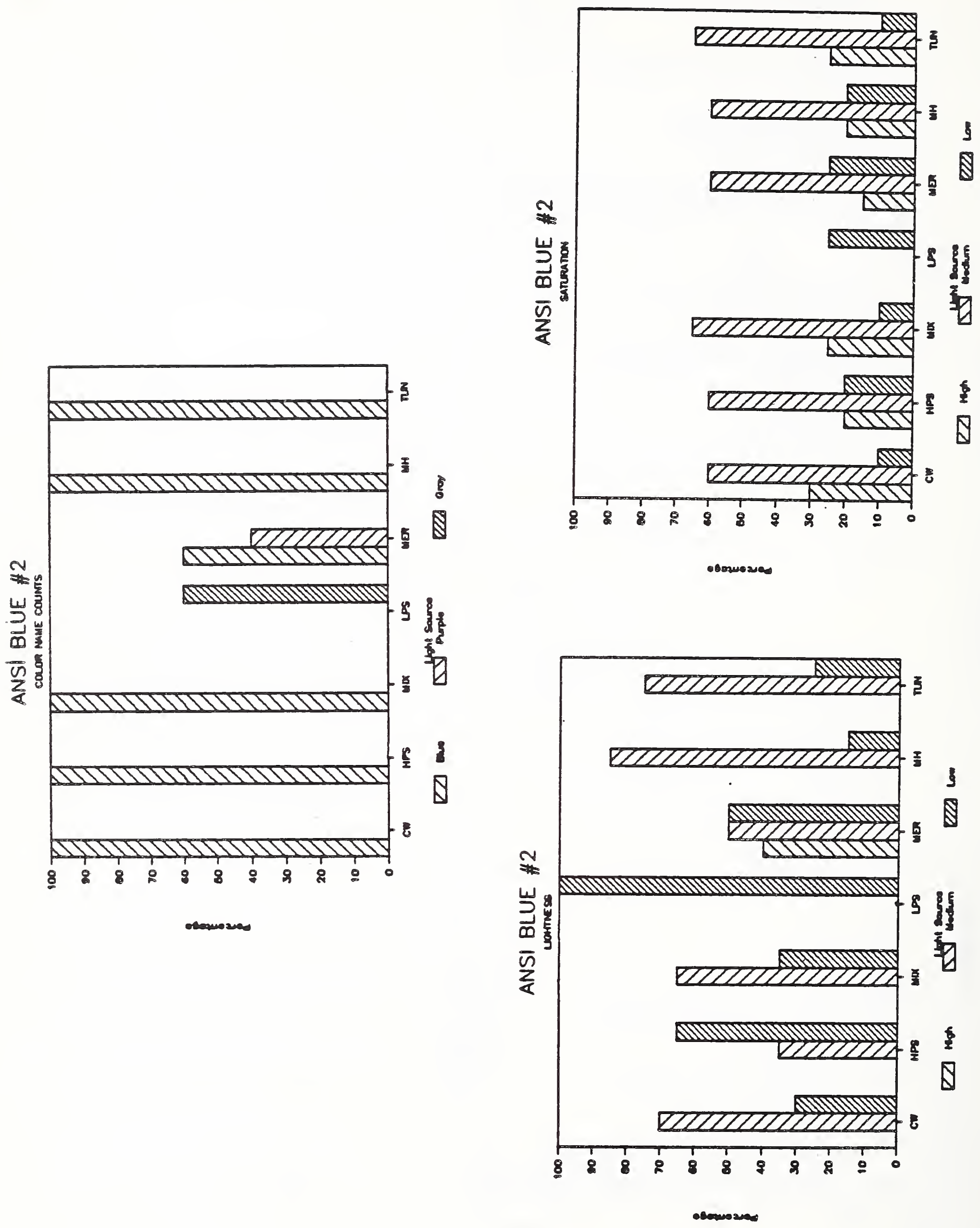

Figure 19 Color naming, lightness, and saturation data for ANSI blue. 

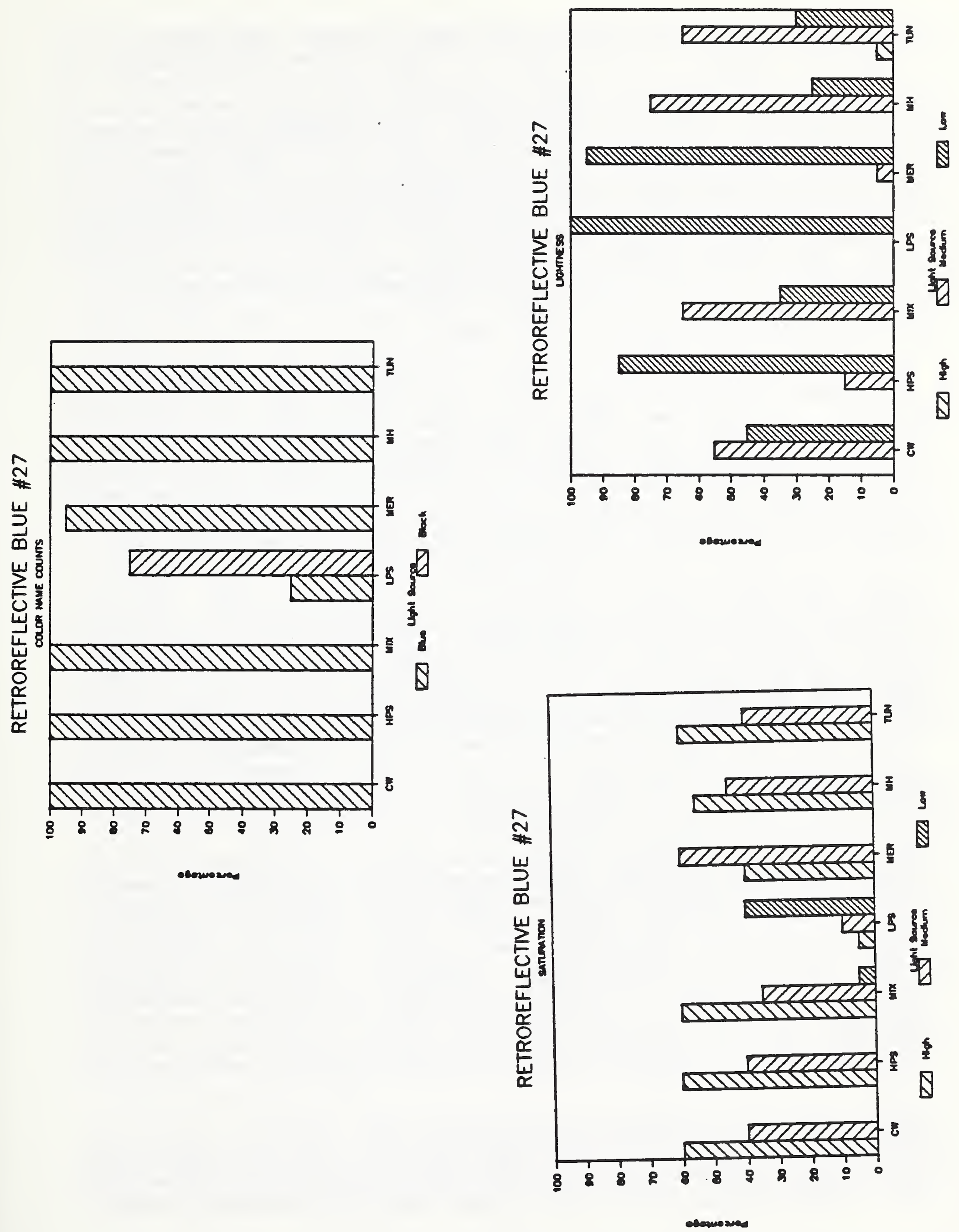

Figure 20 Color naming, lightness, and saturation data for sample \#27 (highway blue). 
The reanalysis of the Collins et al. data indicates that highway yellow was significantly less accurately recognized than ANSI yellow with numerous confusions with orange as well as lower saturation and lightness. In addition, both the highway green and blue were seen as darker than the comparable ANSI color. While highway red was generally recognized more accurately than ANSI red, its performance was equally poor under HPS, where both colors were termed orange. The widespread use of HPS for roadway lighting and the importance of red in signalling stop and prohibited actions suggests that there is the potential for serious confusions with the highway specifications for this color. On the other hand, the analysis suggests that highway orange may be more accurately recognized with higher saturation and lightness than ANSI orange.

\subsection{Other Studies of Highway Sign Colors}

MacNeill (1965) evaluated the impact of different color combinations on observers' ability to detect caution and warning signs for three light levels. He determined that black on yellow, and white on black were significantly more legible than combinations such as white on red, red on black, or black on red. In addition, white on orange and white on red were completely illegible under low red illuminance, while red and black were illegible under low white illuminance. As a result, he recommended using black on yellow for caution signs for maximum legibility - a practice currently followed by both ANSI and FHWA.

Olson (1988) evaluated the conspicuity of road sign materials. Following Cole and Jenkins (1982), he defined a conspicuous object as "one that will, for any given background, be seen with certainty $p>90 \%$ within a short observation time ( $t=250 \mathrm{~ms})$ regardless of the location of the object in relation to the line of sight" (Olson, 1988, p.1). Pointing out that this definition is an operational one which specifies both a response level and operating conditions, he defined adequate conspicuity as yielding $85 \%$ or better correct identification at appropriate distances from the sign, with the 85 th percentile response criterion being used because that is common in U.S. practice.

The purpose of Olson's research was to establish minimum reflectance values for retroreflective signs in settings representing cluttered urban, suburban, and dark rural environments, for people of all ages. To this end, he conducted a field study of the nighttime conspicuity of signs in real world settings for two age groups (20-46 and 58-75). The signs varied in retroreflective properties, color, and background complexity. Subjects in Olson's experiment were driven individually through three test areas, varying in complexity. Their task was to call out the word "sign" when they detected a sign and then name its color once they could see that. Distance from the sign and accuracy of the color name were recorded.

Three types of retroreflective materials were studied - cube-corner, encapsulated lens, and enclosed lens with SIA (specific intensity per unit area or coefficient of retroreflection) values ranging from 11 to 750. Signs in all colors, but not all SIA's were studied in each of 
three complexity levels - high (a busy urban thoroughfare), medium (less complex suburban street), and low (a two-lane rural road).

Olson found that sign identification distance varied as a function of SIA and surround complexity, with distance decreasing with complexity but increasing with SIA, at least for yellow signs. The results indicated that higher SIA's were associated with greater sign identification distances, and that increasing background complexity decreased sign conspicuity. Using more highly reflective materials decreased the effect of complexity, however. Performance for older subjects indicated that they needed signs with an SIA about three times greater than younger subjects. Olson found that yellow signs were identified correctly about 908 of the time, but significant confusions arose between red and orange, orange and yellow, and blue and green. Again older subjects made somewhat more errors and had shorter sign identification distances.

Olson found that colored signs other than yellow had greater sign identification distances than yellow signs with about the same SIA, except for a white sign located in an area with a great deal of white. 7 "However, the sign identification distances associated with the red, orange, green, and blue signs were all substantially greater than those for yellow signs having approximately the same SIA. This result was very much unexpected." Olson attributes this difference to the same phenomenon that underlies heterochromatic brightness matching in which colored lights equivalent in luminance to white lights are seen as brighter (Howett, 1986). Consequently, Olson conducted a laboratory study in which red, orange, green, and blue colors were judged to be brighter than yellow. Olson commented that heterochromatic brightness may not be the sole explanation and that red, orange, green, and blue may have inherently greater conspicuity in the context of road signs. Nevertheless, his results indicated strongly that conspicuity depends significantly on sign color as well as SIA (or brightness).

Olson also developed a correction for expectancy to overcome the fact that subjects were expecting to see signs and to make the task more realistic. This meant that the distances measured in the field study were reduced by about $40 \%$ to compensate for driver expectancy. Based on these data, Olson provided recommended SIA values for stop, construction, overhead guide, and warning signs. Making recommendations for SIA was complicated by the interaction with sign color since olson had not anticipated the strong color effect and had not studied all possible color/SIA/complexity conditions. Olson's data reinforce the need to conduct experiments on the detectability of colored retroreflective materials under both diffuse (daylight) and directional (nighttime) viewing conditions. They make it clear that color contributes substantially to the conspicuity of sign materials.

7 Olson did not address the issue of yellow hazard-warning signs which may have been located along the same route followed by his observers. Observers may have had trouble discriminating experimented yellow signs from real yellow signs. 


\section{MEASUREMENT OF RETROREFLECTIVE COLORS}

In this section measurements of the chromaticity and luminance of currently available retroreflective highway safety colors (as provided by five manufacturers) are given in both CIE $\mathrm{x}, \mathrm{y}$ and CIELAB color space. The impact of different instruments, measuring geometries, and illuminants is discussed. The feasibility of developing a nighttime color standard is also addressed.

\subsection{Background on Retroreflection}

Shinar (1985) conducted a study in which both the expectancy of seeing a pedestrian at night and the reflection of the pedestrian's clothing were varied. Although the use of retroreflective and high visibility materials increases pedestrian detectability, the reduction in accident frequency is smaller than expected perhaps because drivers do not expect to see pedestrians and do not respond appropriately. In Shinar's experiment, 40 observers were instructed to detect pedestrians on a roadway under four different expectancy conditions. In the first condition, the observer had no awareness of where the pedestrian would be in the roadway, while in the fourth condition the car was stationary, and the pedestrian walked away from it until the observer could no longer detect him. The other two conditions were intermediate in the amount of knowledge provided about the pedestrian's probable location. The second variable was clothing reflectance, including dark to light khaki, and dark khaki plus a retroreflective tag. In addition, for one condition, observers were instructed to look for a moving retroreflective tag (which would indicate the presence of a pedestrian). The results indicated that visibility distance increased significantly for the four expectancy and clothing conditions. There was a significant interaction between expectancy and detection distance for the retroreflective tag. The results also indicated that if a driver expected to see a pedestrian, light clothes would increase his detectability slightly, but use of a retroreflective tag increased his detectability very significantly. The retroreflectivity was effective, however, only if the driver associated it with the presence of a pedestrian.

Olson and Bernstein (1979) evaluated the nighttime legibility of highway signs. A laboratory study was conducted in which seven colors (yellow, orange, white, red, blue, green, and black) were used as backgrounds at different background luminances from $0.07 \mathrm{~cd} / \mathrm{m}^{2}$ to $211 \mathrm{~cd} / \mathrm{m}^{2}$. Sign legend was black for the first three colors listed above and white for the last four colors (at a luminance of 0.03 to $737 \mathrm{~cd} / \mathrm{m}^{2}$ ). Two surround luminance conditions were used; high $\left(5.14 \mathrm{~cd} / \mathrm{m}^{2}\right)$, and low $\left(0.02 \mathrm{~cd} / \mathrm{m}^{2}\right)$. The lower luminance condition approximated the illumination provided by low beam headlights in a car. The sign legend consisted of a Landolt ring in different orientations. Three young adults participated in all viewing conditions; five older (65+) adults participated in a partial set; and three other young adults with poorer low contrast acuity also participated in a partial set. Data for the white and green sign condition for young adults indicated that more legend contrast was needed as background luminance decreased (and as viewing distance increased). 
Data for blue and green backgrounds were similar. For red backgrounds, legend contrast had to be increased by as much as 1.7 times for luminances of $3.77 \mathrm{~cd} / \mathrm{m}^{2}$ and below. The data for black backgrounds were similar to those for colored backgrounds at the lowest luminance levels suggesting that the effects of color contrast effects become ineffective at luminance levels between 0.3 and $0.03 \mathrm{~cd} / \mathrm{m}^{2}$.

The authors commented that their data provide evidence of systematic differences in the luminances required for recognition accuracy at different background colors. Thus, white, orange, and yellow required the lowest luminances (in that order) for a given level of performance. Increasing surround luminance increased legibility distance by about 510 percent. The authors also found that subjects with poorer contrast acuity required greater luminance contrast - sometimes as much as ten times as much - than the normal group. Of interest is the much greater error rate of the older subjects, regardless of luminance contrast.

Olson and Bernstein then conducted a field study in which they first evaluated the distance at which subjects could determine the orientation of the letter "E" placed on white and green signs varying in both background and legend reflectivity. Eighteen subjects indicated the distance at which they could determine the position of the "E" on the sign. In a second study, the same subjects indicated when they could read the legend on 23 fully reflectorized green and white signs located along a section of actual freeway.

Data from both studies enabled the authors to develop a visibility model for predicting field legibility distances. This model states that legibility distance will increase somewhat with increasing background reflectivity. Fully reflectorized signs have a distinct advantage in legibility distance because of their greater legend/background luminance contrast. The authors appears to have assumed that switching from low to high beam headlights with retroreflective backgrounds does not increase background reflectance but does increase legend luminance so that the contrast of the sign increases. They do not provide any measurements of contrast, however. Studies demonstrating that retroreflective materials can be detected more rapidly and accurately have led to the specification of retroreflective materials for nighttime highway use. An important research issue is that of measuring the effectiveness of the different types of retroreflective materials under field conditions - both in chromaticity and in luminance.

\subsection{Measurement of Retroreflective Materials}

Lozano (1980) pointed out that it is not likely that the color of retroreflective signs will be the same under daytime diffuse, and nighttime directional viewing conditions. The results obtained for safety color appearance under different illuminants indicate that the problem of accurate color identification is even greater when HID illuminants are used (Jerome, 1976; Collins et al., 1986). Lozano commented that the color of retroreflective material should appear about the same both day and night (for illuminants such as CIE $A$ and $C$ ) even 
though the measured chromaticities may well have shifted. He also noted that the legibility of a sign can be reduced by 30 percent or more by poor maintenance.

In a monograph which reviewed the state of the art in laboratory measurements of retroreflectivity, Eckerle (1980) pointed out that retroreflectors are illuminated diffusely during the day, while at night they are illuminated directionally by a small source near the observer. He defined the entrance angle, $\beta$, as the angle between the reference axis and the illumination axis, and the observation angle, $\alpha$, as the angle between the illumination axis and the observation axis. The observation distance is the distance between the retroreflector reference center and the receptor (measuring instrument) aperture (or observer). Setting the accuracy of the observation angle is critical and can cause substantial changes in CIL (Coefficient of Luminous Intensity) and chromaticity with variations in blue samples as high as 118 between laboratories. Eckerle also pointed out that the color boundary specifications for retroreflectors must take into consideration the fact that the color of a retroreflector will vary with geometry from day to nighttime conditions.

Unlike Eckerle, who concentrated primarily on luminance measures for retroreflective colors, Rennilson (1980) presented detailed chromaticity measures for retroreflective materials using different nighttime geometries with a spectroradiometer. In his experiment, retroreflective samples were measured under illuminant A (2856K) provided by a tungsten halogen light source. Chromaticity measures were made for different observation angles $\alpha$ and entrance angles $\beta_{1}$.

Rennilson measured six colors: red, orange, yellow, green, blue, and white in three different types of retroreflective materials - enclosed spherical lens, encapsulated spherical lens, and prismatic material (micro- and macro-cube corner material). While observation angle varied from 0.2 to $2^{\circ}$, and entrance angle varied from -4 to $+50^{\circ}$, Rennilson reported that a $4 \times 4$ matrix containing observation angles $(\alpha)$ of $0.2,0.5$, 1.0 and $2.0^{\circ}$, and entrance angles $\left(\beta_{1}\right)$ of $-4,15,30$ and $50^{\circ}$ was an adequate way of describing the changes in chromaticity with changing measurement geometry. Rennilson discussed these changes in terms of both CIELUV and CIELAB color difference equations, using a reference measuring condition of $0.2^{\circ}$ observation angle and $-4^{\circ}$ entrance angle. His data indicated (personal communication) that changing observation angles (for those angles studied) had less impact on chromaticity than changing entrance angles. Figures given in the paper for blue, green, orange, and red retroreflective samples indicate relatively little change in normalized spectral reflectance factor as a function of changing observation angle, particularly for those angles below $1.0^{\circ}$.

Asher, Harrington, and Stephenson (1978) reported an international intercomparison from 10 laboratories of chromaticity and photometric measurements of retroreflective materials. Fifteen samples of retroreflective sheeting, both enclosed and encapsulated lens type, were measured for white, red, orange, yellow, green and blue. (A larger set of 44 samples were measured under nighttime conditions.) Measurements 
were made with $45^{\circ}$ incidence and normal view with $45 / 0$ geometry under illuminants $C$ and $D_{65}$ where possible. The measuring instruments were either spectrophotometers or tristimulus colorimeters.

Comparison of the results indicated tremendous variability in the data for chromaticity between laboratories. Spectrophotometers generally had less variability than tristimulus colorimeters. For spectrophotometers, the average spread in chromaticity coordinates was about 0.008 except for a green which had 0.017. For tristimulus colorimeters, the average chromaticity coordinate spread was about 0.02 , except green where the average was 0.035 . The luminance factors also varied with an average spread of $15 \%$ for whites, yellow, oranges, and reds, and $23 \%$ for greens and blues.

The authors commented that "As was found from the nighttime results, spectrophotometric methods correlated with each other better than do the tristimulus measurements. However, on some samples, spectral measurements gave a variability similar to that of the tristimulus readings. In general, the variability in chromaticity measurements made with both day- and nighttime geometries was of a similar order which indicates that differences in colorimetric technique are an important cause of the variability" (Asher et al., 1978, pp.27-28).

\subsection{Comparison of Chromaticity for Retroreflective Color Samples}

For the present paper, the chromaticity of 88 retroreflective samples from seven different manufacturers were measured using different measuring instruments and measurement geometries. Three types of materials were used - engineering grade, super engineering grade, and high intensity. Although luminance factors were measured, the primary focus was on a determination of the extent in chromaticity shifts as a function of directional viewing and illuminant to provide some indication of shifts to be expected under nighttime viewing conditions.

Measurements were made initially in the NIST color laboratory for three measuring devices - a Minolta colorimeter ${ }^{8}$ CR 231 with internal Illuminant $C$, a Photo Research SpectraScan Spectroradiometer with Illuminant A, and a Minolta spot colorimeter CS-100, also with Illuminant A. All instruments were used with $45 / 0$ illuminating and viewing geometry. A second series of measurements was made under directional viewing conditions in the NIST radiometry tunnel (described by Eckerle, 1980) using the spectroradiometer with source $A, a-4^{\circ}$ entrance angle, and a $0.6^{\circ}$ observation angle. The radiometry tunnel was used because it was long enough to allow the detector to be placed near the illuminant. Although this tunnel was designed for precise measurements of retroreflectors, the specialized instrumentation in place in it measures only the luminance, not the chromaticity, of retroreflectors. As a result, the normal photometer could not be used, so the spectroradiometer

8 Brand names are provided for identification purposes only and do not constitute endorsement by NIST or FHWA. 
was substituted, with less precise positioning. Ideally, an entrance angle of $0.2^{\circ}$ would have been used for the measurements but even at a distance of $35 \mathrm{ft}$ in the tunnel, the smallest angle at which the spectroradiometer detector was properly filled was $0.6^{\circ}$. Increasing the distance, to reduce the entrance angle, meant that the field of view on the detector was underfilled.

All the samples were measured under illuminant A, with the corresponding white retroreflective sample used to determine the spectral coefficient of retroreflection for subsequent calculations. The chromaticity and luminance for each sample were then calculated for illuminant $C$ after dividing out the effects of the measuring illuminant using the program ADNK (Collins and Worthey, 1984; Collins et al., 1986). Comparisons were made for illuminants $A$ and $C$ because $A$ represents nighttime illumination conditions, while C represent daytime illumination. In addition, comparisons were made between directional and non-directional viewing conditions, again to represent nighttime and daytime conditions.

Figure 21 presents the chromaticity measurements in CIELAB for three different measuring procedures for a set of engineering grade samples. This graph compares measurements taken under directional viewing conditions with those taken under $45 / 0$ conditions, as well as those taken with the spectroradiometer and Minolta colorimeter under 45/0. In this graph, the squares (and subscript 1) represent measurements made with the spectroradiometer using directional geometry in the radiometry tunnel, the pluses (and subscript 2) represent measurements made in the color laboratory with the spectroradiometer using 45/0 geometry, and the diamonds (and subscript 3) represent measurementss made with the Minolta CR 231 colorimeter also using 45/0 geometry. All comparisons are for illuminant $C$. In this and subsequent figures, $R$ refers to the red sample, $O$ to orange, $Y$ to yellow, $G$ to green, $B$ to blue, and $W$ to white.

Although the two sets of measurements taken in the $45 / 0$ mode (subscripts 2 and 3 ) with the spectroradiometer and the colorimeter might have been expected to coincide, they did so only for the blue sample. Measurements in the directional mode should not have been expected to coincide with those in the 45/0 mode, and they did not, except for the white sample. The variability between the various instrument readings was greatest for the yellow and least for the orange, but there was no consistent pattern for the different measuring conditions. The effects of variation in lighting geometry were greatest for the blue sample.

Figure 22 presents two graphs for the same samples shown in figure 21 , showing the shift in $a * b *$ space as a function of switching from source $C$ (daytime viewing) to source A (nighttime viewing). In both portions of this figure, all measurements were made with the spectroradiometer. The upper graph compares the data for directional viewing conditions (those obtained in the tunnel), while the bottom graph presents data for 45/0 geometry (obtained in the color lab). In this figure, data for source $A$ are represented by squares, while those for source $C$ are represented by pluses. Subscripts 1 and 2 refer to source $C$; subscripts 4 and 5 refer to source A. 


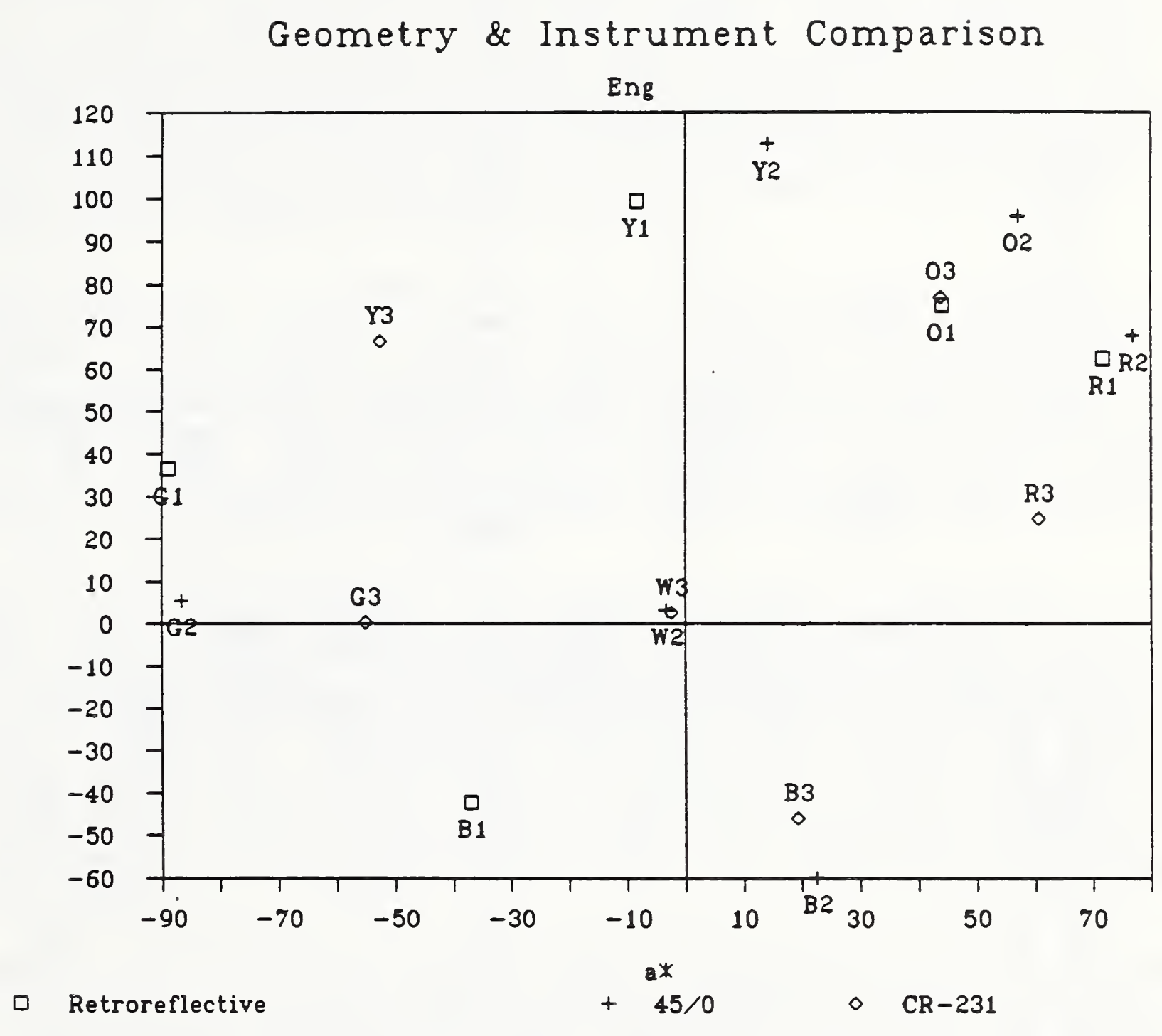

Figure 21 Instrumental Comparisons for Engineering Grade Samples for
Source C. 
Comparison of Source C with Source A

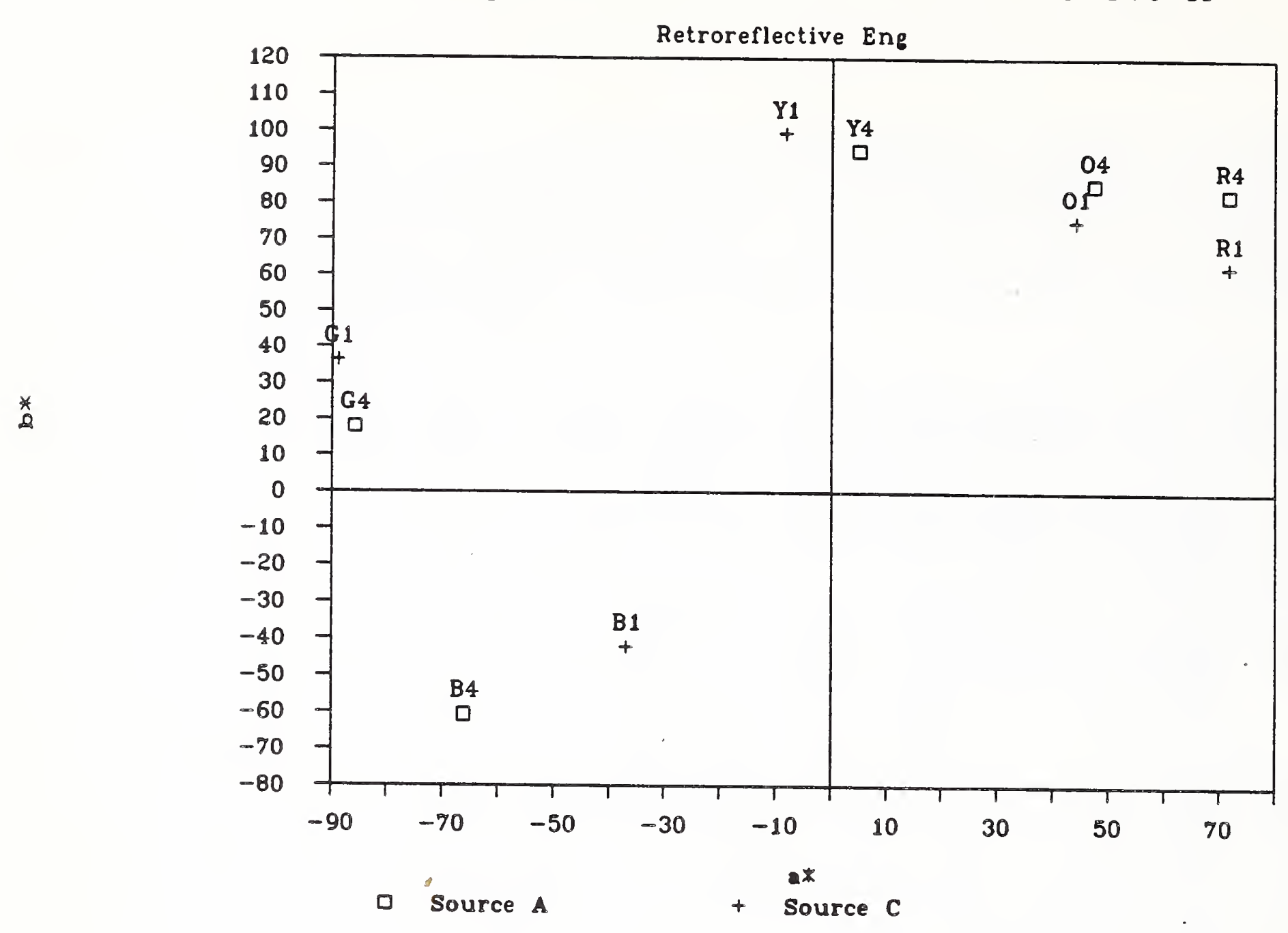

Comparison of Source C With Source A

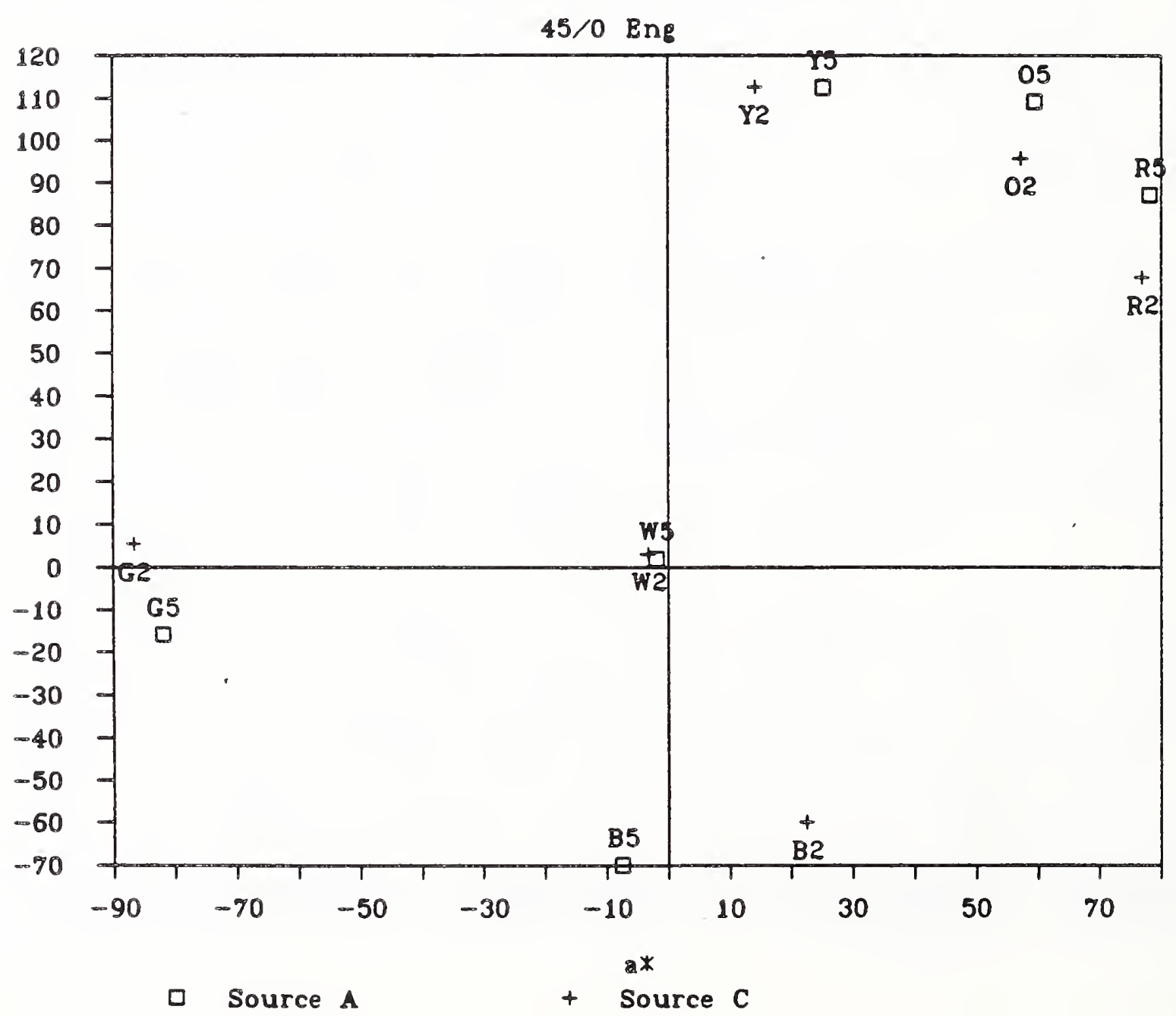

Figure 22 Shift from Illuminant C to Illuminant A for Retroreflective and 45/0 Viewing Conditions for Engineering Grade Samples. 
Two points can be made about the graphs in figure 22. Comparing the upper graph with the lower graph demonstrates that the geometry (directional vs. 45/0) under which the samples were measured shifted their position in $a * b *$ space substantially, with the greatest shift occurring for blue samples. Secondly, comparing the individual data suggests that the chromaticity of all the samples except white shifted somewhat as the illuminant was changed from source $C$ to source A. Again the shift was greatest for the blue sample. (Some of this shift may be due to the mathematical assumptions for CIELAB and the von Kries transformation or to noise in the spectroradiometer at low light levels in the short wavelength portion of the spectrum.) Comparison of the two graphs indicates that the way in which the sample was measured - 45/0 versus directional - had as much or more impact on its position in color space as did changes in the illuminant.

Figure 23, which presents data for a set of super engineering grade retroreflective samples, shows the same variability between measurement methods. The greatest scatter between methods occurred for the yellow sample, and the least for the orange. Again, the data obtained with the Minolta 231 were different from those obtained with the spectroradiometer for all samples except the blue and orange. These data reinforce the idea that the geometry used for measuring the samples has a marked impact on their position in a*b* space.

Figure 24 presents graphs comparing the shift in chromaticity for the same set of super engineering grade samples for sources $A$ and $C$. The upper graph presents the directional (retroreflective) measurements made in the tunnel and the lower one presents $45 / 0$ measurements made in the color laboratory. Both graphs show appreciable shifts due to the light source, with the greatest shifts occurring for the blue sample. Again, comparing the two graphs reveals that the measurement technique had a marked effect on the position in $a * b *$ space, particularly for green and blue samples.

Figures 25 and 26 present similar data showing the shifts in chromaticity for sources $A$ and $C$ for samples from two other manufacturers, with figure 25 representing engineering grade samples, and figure 26 representing high intensity grade samples. As in figure 24, the upper graph presents directional measurements, while the lower one presents $45 / 0$ measurements. Both figures demonstrate that the use of different measuring techniques clearly shifted the samples in $a * b *$ space as much as the shift from source $A$ to $C$. Despite the variability in chromaticity due to measuring geometry and instrument, color samples from different manufacturers tended to plot in about the same place in CIELAB space for the same measuring technique and illuminant. This suggests that there was consistency in the measuring technique for a given geometry and instrument. 
Geometry \& Instrument Comparison

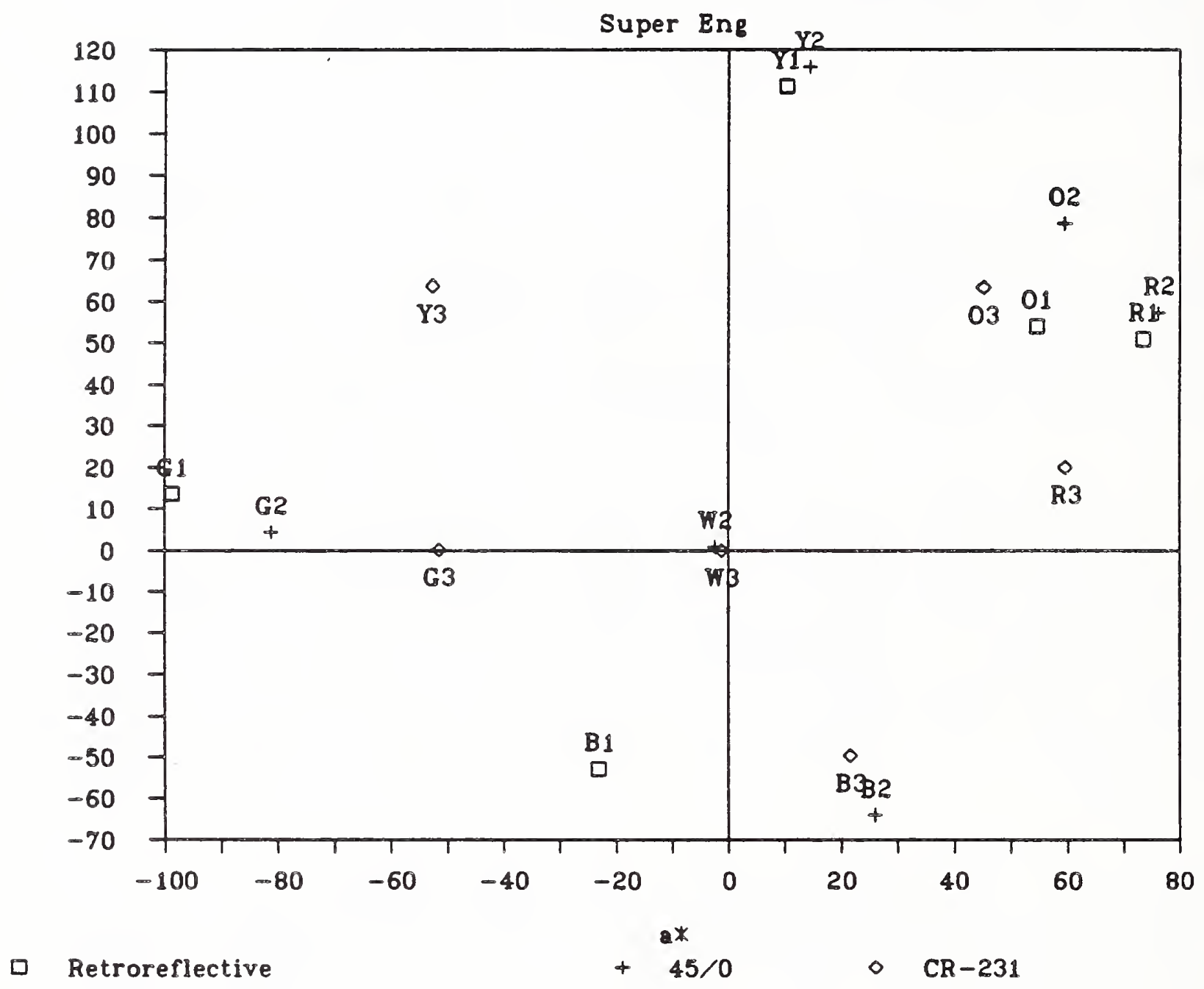

Figure 23 Instrumental Comparisons for Super Engineering Samples. 
Comparison of Source C With Source A

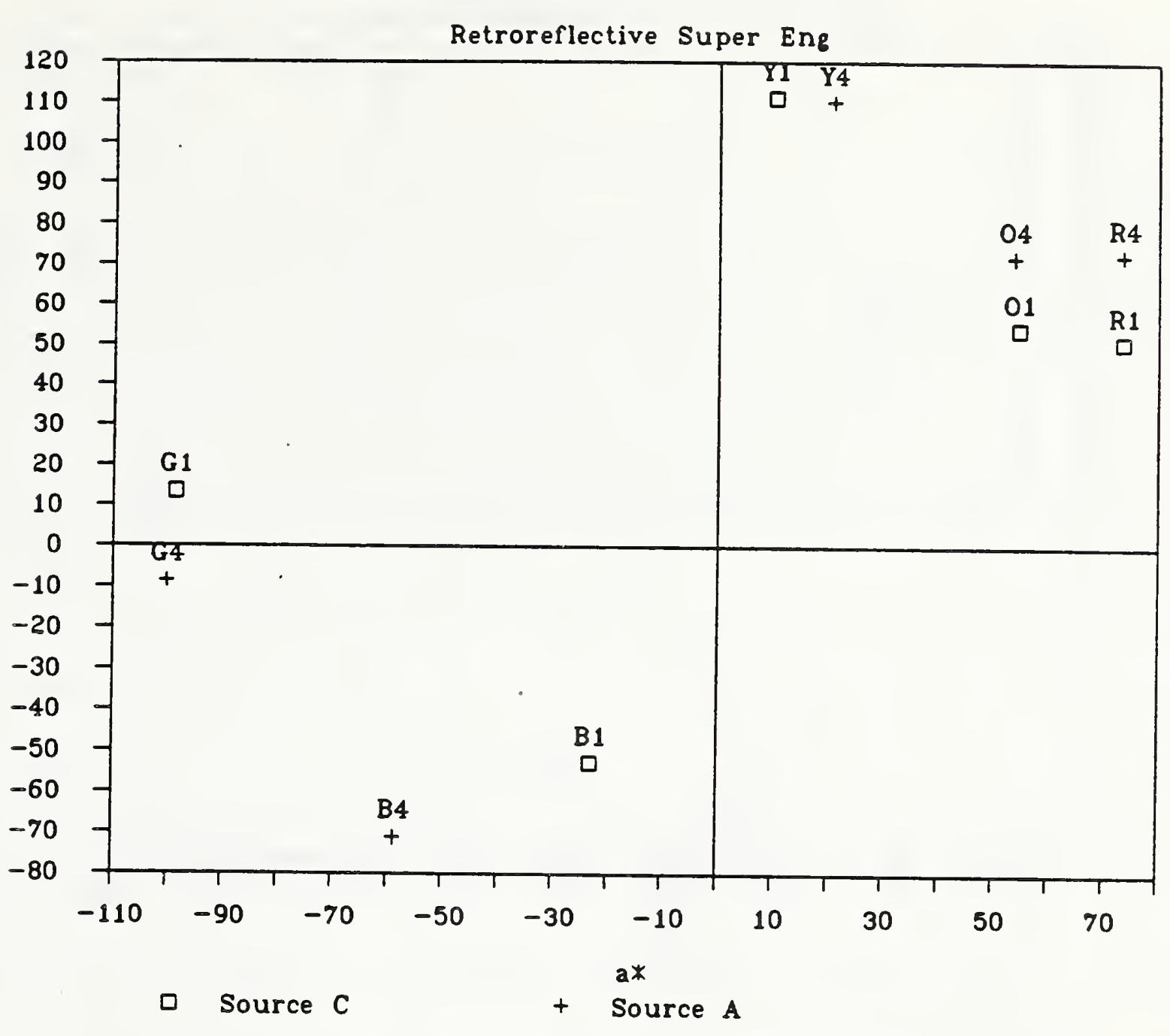

Comparison of Source C With Source A

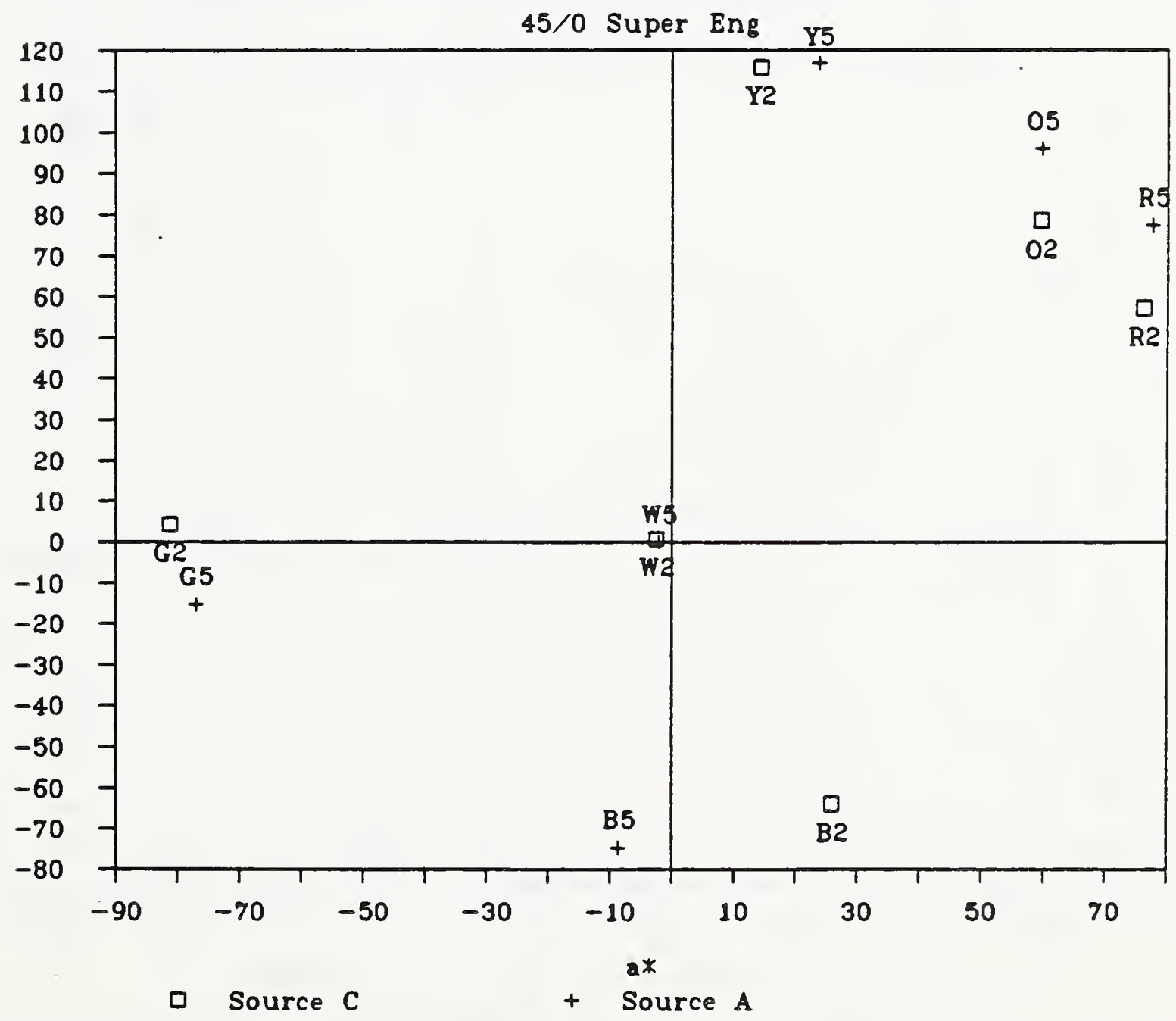

Figure 24 Shift from Illuminant C to Illuminant A for Retroreflective and 45/0 Viewing Conditions for Super Engineering Samples. 
Comparison of Source C With Source A

巻

Retroreflective Eng

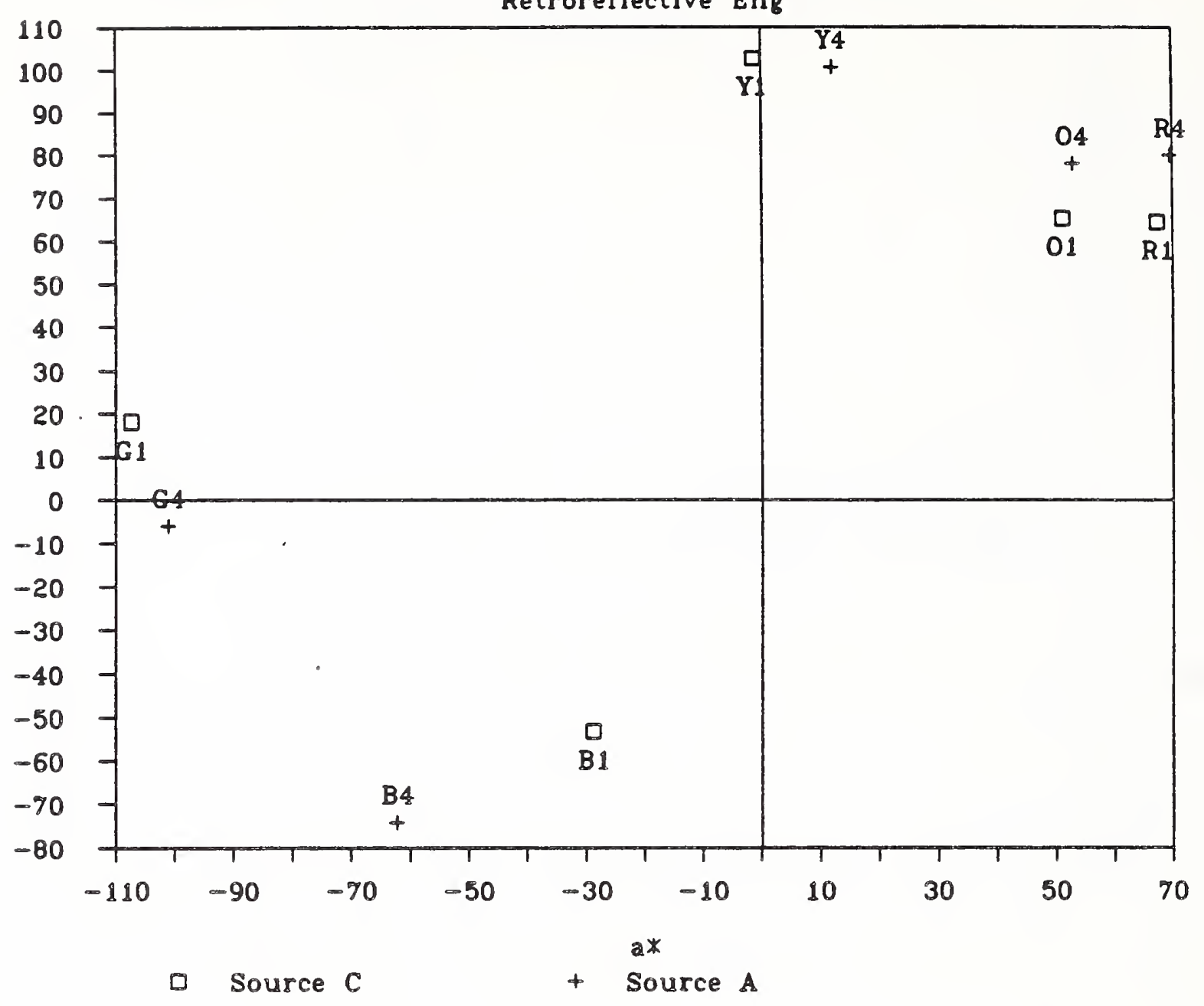

Comparison of Source C With Source A

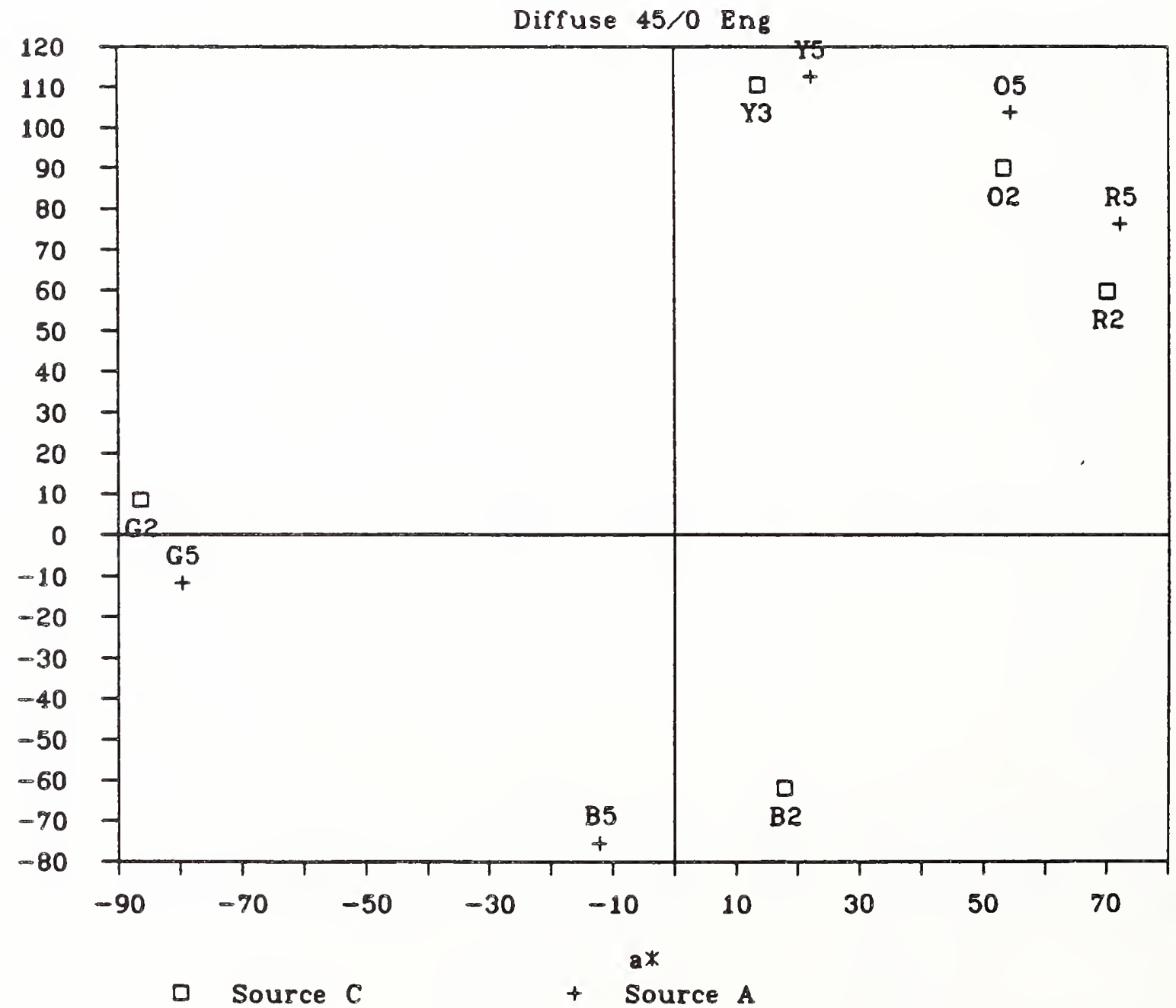

Figure 25 Shift from Illuminant $C$ to Illuminant $A$ for Retroreflective and 45/0 Viewing Conditions for Engineering Grade Samples. 
Comparison of Source $C$ With Source A

*

Retroreflective High Intensity

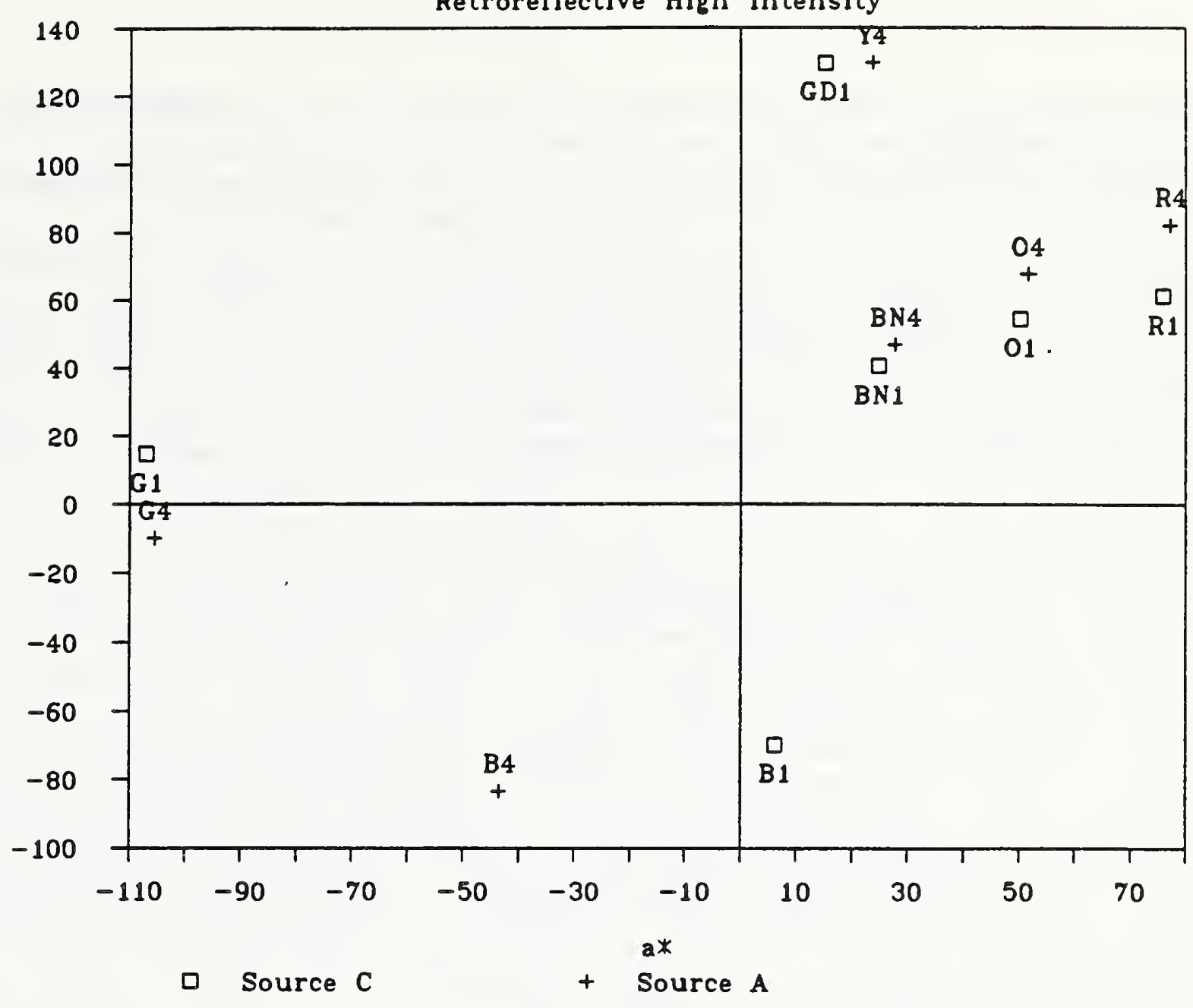

Comparison of Source C With Source A

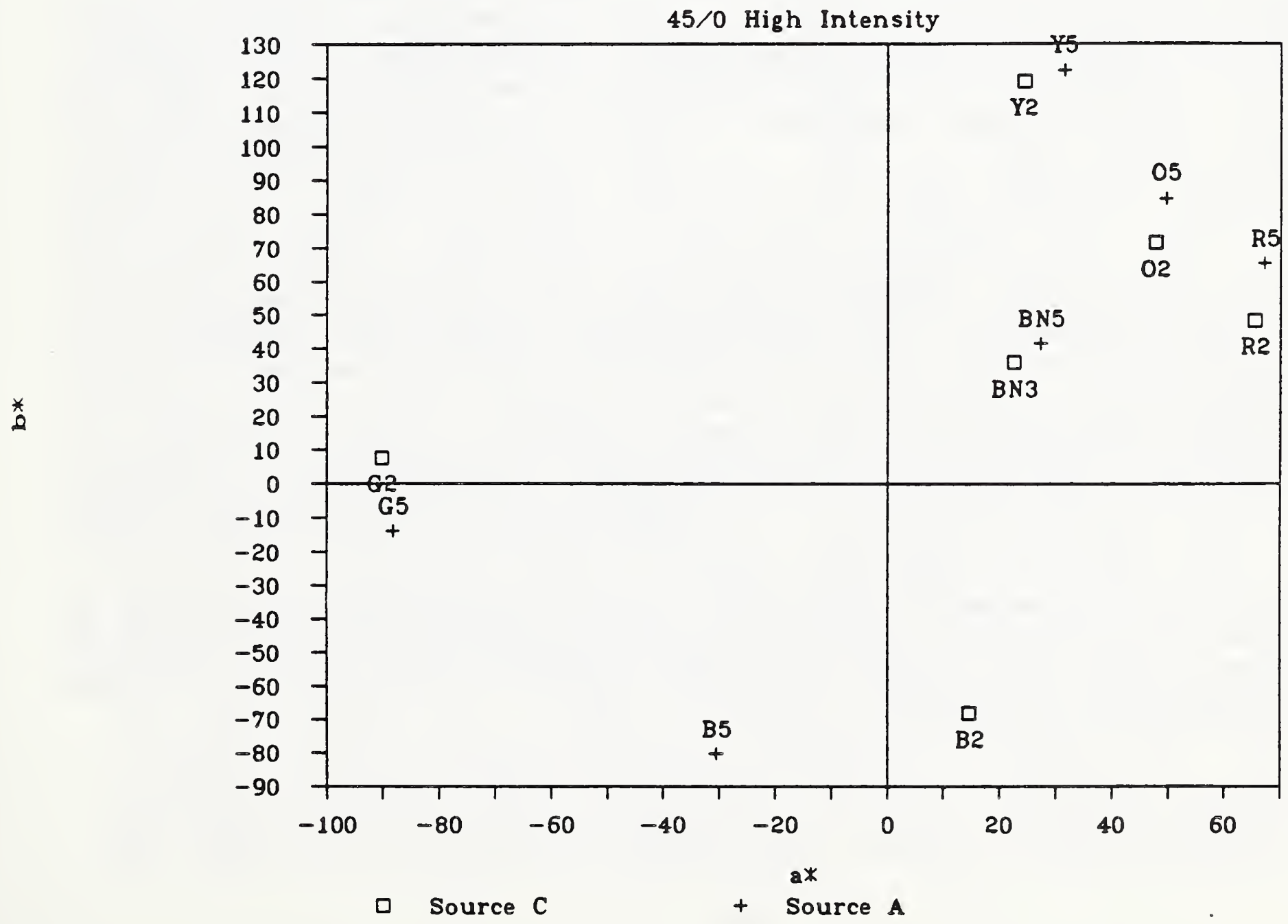

Figure 26 Shift from Illuminant $C$ to Illuminant A for Retroreflective and 45/0 Viewing Conditions for High Intensity Samples. 
Nevertheless, the data presented in figures 21-26 underscore the difficulty of making accurate measurement of the chromaticity of retroreflective sign material even under controlled laboratory conditions. They demonstrate that the use of a self-contained instrument such as a Minolta CR231 does not provide the same results as a spectroradiometer under either 45/0 geometry or directional geometry. Nor do the latter two measuring techniques agree with each other.. In many cases, the chromaticity shifts due to measuring technique are beyond the color tolerance limits for the highway samples given in Table 1 - not because the sample changed, but simply because the way in which it was measured differed.

Such variability reinforces the difficulty of developing a simple field procedure for measuring the color of retroreflective sign material. Because changing from 45/0 geometry to directional measuring conditions appears to affect chromaticity substantially, short cut techniques using non-directional lighting conditions (such as those provided by the Minolta 231) will not be of sufficient accuracy to determine whether a sign meets the retroreflective color specifications used by FHWA. An attempt to use a car headlamp as an illuminant in a field method was not particularly successful due to the need to maintain precise alignment between the detector and the illuminant. A field technique is proposed in appendix $A$ which would use an instrument especially designed with an integral light source for making directional measurements but this requires validation before being used.

Figures 21-26 suggest that using different measuring instruments may have had less impact on the chromaticity measurements than switching viewing conditions from $45 / 0$ to retroreflective. These graphs also indicate, however, that the switch from illuminant $A$ to illuminant $C$ shifts the colors in color space, sometimes out of the color tolerance limits for highway colors given in table 1 . This suggests that the measurement techniques followed here do not allow one to assume that daytime chromaticity and nighttime chromaticity are the same. Rather, even under the less-than-ideal laboratory conditions used, in which the geometry was similar to, but not identical with, that currently recommended by the CIE, the chromaticity of retroreflective material was different for Illuminants $A$ and $C$. This indicates that one cannot measure a sample under daylight conditions and assume that these measures will meet the nighttime specifications. The solution appears to be the use of a field measurement technique such as that presented in Appendix A, or rewriting the specifications for daytime illumination and calculating the likely shift for nighttime conditions. The data obtained to date make it very clear that there are appreciable shifts in the chromaticity of retroreflective samples between illuminants $C$ and A. Much greater shifts can be expected for HID sources such as HPS and clear mercury, reinforcing the contention that the daytime and the nighttime color of current sign materials are not the same. 


\section{CONCLUSIONS}

\subsection{Feasibility of Altering Current Highway Color Specifications}

The detailed reanalysis of the data collected by Collins et al. for safety color appearance indicates strongly that highway yellow was significantly less accurately recognized than ANSI yellow, with more confusions with orange, lower saturation, and lower lightness. In addition, both highway green and blue were seen as darker than the comparable ANSI color. On the other hand, highway orange was more accurately recognized with higher saturation and lightness than ANSI orange. While highway red was generally recognized more accurately than ANSI red, performance for both was very poor under HPS (and LPS) where they were termed orange. The widespread use of HPS as a roadway illuminant and the importance of red to indicate "stop" and prohibited actions suggests the potential for serious confusions for this color. Nonetheless, the reanalysis of the data on safety color appearance suggests very strongly that serious consideration should be given to altering the AASHTO (FHWA) specifications for highway colors to meet the ANSI specifications. Such a move should increase the general recognizability of colors for traffic signs, and increase the unity among standards for safety colors in the United States.

Because the ANSI colors are widely used in industrial and other safety applications, there should be no problem with using these colors on ordinary highway signs. While there have been problems in the past with making retroreflective colors with higher luminance factors, analysis of the chromaticity data for the samples obtained from several different manufacturers suggests that at least some are close to the ANSI samples, indicating that the feasibility is greater now. (Obviously, of course, currently available retroreflective materials meet the AASHTO, not the ANSI specifications.)

\subsection{Additional Research}

Before specifications are changed dramatically, though, there is a need for further research on the recognizability of retroreflective colors viewed under directional lighting. The data collected by Collins et al. were only for color samples viewed by diffuse illumination. While this represents a major portion of highway viewing, it does not deal with the nighttime situation of signs illuminated by headlights and HID lamps. The colors identified as more effective should be evaluated under nighttime viewing type conditions.

Ideally the evaluation would be done as a psychophysical investigation under laboratory conditions in which geometry, illuminant, and adaptation level are precisely controlled and then validated under field conditions. The study should use a range of observation and entrance angles, which simulate those seen by a motorist approaching a highway sign. A baseline condition under diffuse overhead "daylight" illumination should be included to provide data on the extent of color appearance shifts due to the shift from diffuse to directional viewing. Color samples should 
include retroreflective versions of the ANSI colors and the current FHWA colors to verify that the data from Collins et al. (showing better performance by the ANSI colors) are in fact valid for retroreflective viewing conditions. Color samples should also include aged materials, as well as different types of retroreflective materials from engineering grade to high intensity. The adaptation level should approximate that of a nighttime driver, although the illuminant on the retroreflector should be at normal headlamp intensity. The illuminants should include tungsten halogen, metal halide, and high pressure sodium since the latter sources are candidates for car headlamps in the future. The experimental procedure should determine dominant color name, primary and secondary hue, lightness, and saturation for each color sample to maximize information on color appearance shifts. At the same time, physical measurements should be made of the chromaticity and luminance of each sample under each viewing condition, so that the color appearance judgements can be correlated with the physical characteristics of the color samples.

The proposed procedure will provide evidence on the degree of color shift as a function of viewing geometry and illuminant changes. It should ascertain if the apparent improvement in performance for samples meeting the ANSI specifications found by Collins et al. hold for directional viewing conditions. At the same time, the performance of colors which differ from both the ANSI and FHWA specifications can be assessed to determine if there is a "best" color for retroreflective and normal viewing conditions .

Another situation which should be researched experimentally is an evaluation of the chromatic contribution to contrast for colors (as discussed in section 2.1) using surface colors with different illuminants for different combinations of sign colors. In addition, the relative contribution of car headlamps and street/sign lighting should be measured with a spectroradiometer to determine exactly the spectral power distributions that commonly illuminate roadway signs. At the moment, the assumption is that the illuminant is only the headlamp or only the street lighting. The reality is that the illuminant is some unknown mixture of both, which is likely to shift the chromaticity of roadway signs in some unknown manner.

Eventually, the durability of fluorescent pigments particularly for red signs should also be assessed for highway applications. The studies by Glass et al., Collins et al., and Thornton suggest strongly that the best way of ensuring the red and orange are detected as such under HID sources is to make them fluorescent. While some manufacturers claim to make fluorescent materials that perform adequately after several years of aging, the durability of such materials must be evaluated in laboratory and field aging tests, before using outdoors.

Finally, the field measurement technique suggested for measuring the chromaticity of retroreflective colors should be evaluated with an instrument containing the proper light source-detector geometry. Under laboratory conditions, it is relatively easy to get the light source 
close enough to the detector to maintain a very small observation angle. Under field conditions, however, if the light source is separated from the detector, it is virtually impossible to obtain an observation angle of $0.5^{\circ}$, and have the detecting instrument perform adequately. Therefore, a housing for the light source should be designed, built, and evaluated under field conditions to verify the measurements of chromaticity. In addition, another question to be researched is the feasibility of measuring signs under diffuse illumination (daytime) conditions and then calculating the shift in chromaticity for nighttime conditions. This might allow the measurements to be made during the day when it would be safer for the measuring crew. 


\section{RECOMMENDATIONS}

The analysis presented in the preceding pages suggests that there are reasonable grounds for changing the current chromaticity specifications for highway colors (particularly for yellow, green, and blue) to bring them in line with the ANSI Z53.1 (1979) color codes and to increase their detectability for drivers. The data reviewed in section 3 indicate that the ANSI yellow, in particular, is identified more accurately in terms of hue, lightness. and saturation. The reanalysis of the data in section 2 suggests that serious consideration should be given to altering the FHWA specifications for colors used on traffic control devices to meet the ANSI specifications.

While this change is being implemented, however, the effectiveness of the ANSI colors presented in a retroreflective format should be assessed. Such evaluation should involve field and laboratory research to verify that these new retroreflective colors are recognized accurately when viewed under nighttime conditions with both headlights and fixed roadway lighting, as well as under daytime conditions. In addition, the difficulties in measuring retroreflective materials accurately should be explored in greater detail before implementing the field test method given in Appendix A.

Switching to the ANSI 253 specifications would have the additional advantage of bringing all the safety colors specified in the United States into agreement. This would increase the likelihood that people will learn to recognize the colors more readily since all color codes would be the same (See reviews by Christ, 1975; Cole and MacDonald, 1988). The alteration in color specifications should not have any negative impact on the ability of color defectives to recognize the colors, since the ANSI colors were designed to lie off the confusion lines shown in figure 5. While safety colors currently meet the 253 specifications, retroreflective colors obviously do not, requiring time for manufacturers to determine the feasibility of switching retroreflective materials to the ANSI specifications. 


\section{BIBLIOGRAPHY}

AASHTO, Standard Specification for Retroreflective Sheeting for Traffic Control. AASHTO M268-84.

Adams, A.J. Cone pigments and color vision polymorphism. In National Research Council, Committee on Vision, Frontiers of Visual Science: Proceedings of the 1985 Symposium. Washington, D.C. National Academy Press, 1987, pp.125-128.

Alpern, M. Variation in the visual pigments of human dichromats and normal human trichromats. In National Research Council, Committee on Vision, Frontiers of Visual Science: Proceedings of the 1985 Symposium. Washington, D.C. National Academy Press, 1987, pp. 169-193.

American National Standards Institute (ANSI). Safety Color Code for Marking Physical Hazards. New York: ANSI Z53.1, 1979. (Currently being revised as ANSI Z535 198x.)

Asher, L.B., Harrington, T.L., and Stephenson H.F. Colorimetric measurement of retroreflecting materials: II Daytime conditions. Color Research and Application, 1978, 3, pp. 23-28.

ASTM E 808-81. Standard Practice for Describing Retroreflection. Philadelphia, PA: ASTM, 1981.

ASTM E xxx. Draft Standard Practice for Photoelectric Tristimulus Measurement of Object Colors. Currently being considered by committee letter ballot by ASTM, 1988.

ASTM F 923-85. Standard Guide for Understanding the Properties of High Visibility Materials for Individual Safety. Philadelphia, Pa: ASTM, 1985 .

Billmeyer, F. Survey of color order systems. Color Research and Application, 1987,12, p. 173-186.

Billmeyer, F. and Saltzman, M. Principles of Color Technology, 2nd Ed. New York: John Wiley and Sons, 1981.

Birren, F. Safety on the highway...A problem of vision, visibility, and color. American Journal of Ophthalmology, 1957, 43, pp. 265-270.

Booher, H.R. Effects of visual and auditory impairment in driving performance. Human Factors, 1978, 20, p. 307-320.

Boyce, P.R. and Simons, R.H. Hue discrimination and light sources. Lighting Research and Technology, 1977, 9, pp.125-140. 
Boynton, R.M. and Bush, W.R. Recognition of forms against a complex background. Journal of the Optical Society of America, 1956, 46, pp. $758-764$.

Boynton, R.M., and Olson C.X. Locating basic colors in the OSA space. Color Research and Application, 1987, 12, pp.95-105:

Boynton, R.M. Categorical color perception and color rendering of light sources. Proceedings of the CIE Conference, Venice, 1987, pp. 66-69.

Boynton, R.M. Color vision. Annual Review of Psychology, vol. 39, 1988.

Breton, M.E. and Cowan, W.B. Deuteranomalous color matching in the deuteranomalous eye. Journal of the Optical Society of America, 1981, 71, pp. 1220-1223.

British Standards Institute, The Construction of Road Traffic Signs and Internally Illuminated Bollards. BSI 873, part 6. 1983.

Burns, S.A. and Elsner, A.E. Color matching at high illuminances: The color-match-area effect and photopigment bleaching. Journal of the Optical Society of America, 1985, 2, pp. 698-704.

Burns, S.A., Smith, V.C., Pokorny, J., and Elsner, A.E. Brightness of equal luminance lights. Journal of the Optical Society of America, 1982, 72, pp. 1225-1231.

Carter, E.C. and Carter, R.C. Color and conspicuousness. Journal of the Optical Society of America, 1981, 71, pp. 723-729.

Christ, R.E. Review and analysis of color coding research for visual displays. Human Factors, 1975, 17, pp. 542-570.

Code of Federal Regulations, Title 29, Labor Chapter XVII, Occupational Safety and Health Administration, 1981.

Cole, B.L. Visual aspects of road engineering (Paper no. 820). Proceedings of the Sixth Conference Australian Road Research Board. 1972,6 , pp. 102-148.

Cole, B.L. and Jenkins, S.E. Conspicuity of traffic control devices. Australian Road Research, 1982, 12, pp. 223-238.

Cole, B.L. and Vingrys, A.J. Who fails lantern tests? Documenta Ophthalmologica, 1983, 55, pp. 157-175.

Cole, B.L. and Vingrys, A.J. A survey and evaluation of lantern tests of color vision. American Journal of Optometry and Physiological Optics, 1982 , 59, pp. 346-374.

Cole, B.L. and Vingrys, A.J. Do protanomals have difficulty seeing red lights? CIE - Proceedings 20th Session, 1, E04/1-3 CIE Paris, 1983. 
Cole, B.L. and Vingrys, A.J. Are standards of color vision in the transport industries justified? Report to the Australian Department of Aviation, July 1985.

Collins, B.L., Kuo, B.Y., Mayerson, S.E., Worthey, J.A., and Howett, G.L. Safety color appearance under selected light sources. Gaithersburg, MD: National Bureau of Standards, NBSIR 86-3493, December 1986.

Collins, B.L., and Worthey, J.A. The role of color in lighting for meat and poultry inspection. Gaithersburg, MD: National Bureau of Standards, NBSIR 84-2829, March 1984.

Commission Internationale de l'Eclairage (CIE). Recommendations for Uniform Color. Color Difference Equations, and Psychometric Color Terms. Supplement No 2 to CIE Publication CIE $N^{\circ} 15$ (E-1.3.1) 1971/(TC-1.3), 1978.

Commission Internationale de l'Eclairage (CIE). Recommendations for Surface Colors for Visual Signalling. Publication CIE N ${ }^{\circ}$ 39-2 (TC-1.6), 1983.

Commission Internationale de l'Eclairage (CIE). Colorimetry. Publication CIE no. 15 (E-1.3.1) 1971.

Deutsches Institut fur Normung (DIN). Surface Colors for Traffic Signs. DIN 6171, Berlin, June 1970.

Eckerle, K.L. Photometry and Colorimetry of Retroreflection: State-ofMeasurement-Accuracy Report. Washington, D.C.: National Bureau of Standards, Technical Note 1125, July, 1980.

Frome, F.S., Buck, S.L., and Boynton, R.M. Visibility of borders: Separate and combined effects of color differences, luminance, contrast, and luminance level. Journal of the Optical Society of America, 1981, 71, pp. 145-150.

Glass, R.A., Howett, G.L., Lister, K., and Collins, B. L. Some Criteria for Colors and Signs in Workplaces. Washington D.C.: National Bureau of Standards, NBSIR 83-2694, April 1983.

Green, B.F. and Anderson, L.K. Color coding in a visual search task. Journal of Experimental Psychology, 1956, 51, p.19-24.

GSA, Instrumental Photometric Measurements of Retroreflective, Materials and Retroreflective Devices, Federal Test Method Standard LS-300C, March $1,1977$.

Halsey, R. M. Identification of signal lights. I. Blue, green, white, and purple. Journal of the Optical Society of America, 1959a, 49, p. 4555 . 
Halsey, R. M. Identification of signal lights. II. Elimination of the purple category. Journal of the Optical Society of America, 1959b, 49, p. 167-169.

Howett, G.L. Linear Opponent-Colors Model Optimized for Brightness Prediction. Gaithersburg, MD: National Bureau of Standards, NBSIR 853202, February 1986.

Hurvich, L.M. Color vision deficiencies. In D. Jameson \& L.M. Hurvich (Eds.) Handbook of Sensory Physiology, Vol 7/4, Visual Psychophysics. Berlin: Springer Verlag, 1972, Chap.23, pp. 582-624.

Hurvich, L.M. Color Vision. Sunderland, Massachusetts: Sinauer Assoc., 1981.

Illuminating Engineering Society (IES). IES Lighting Handbook, Reference Volume. New York, New York: Illuminating Engineering Society of North America, 1984.

International Organization for Standardization (ISO). Safety Colors and Safety Signs. ISO 3864.1984-03-01.

Jacobs, G.H. Cone pigments and color vision polymorphism. In National Research Council, Committee on Vision, Frontiers of Visual Science: Proceedings of the 1985 Symposium. Washington, D.C. National Academy Press, 1987, pp. 129-144.

Jacobs, G.H. Cones and opponency. Vision Research, 1986, 26, pp. 15331541.

Jacobsen, A.R., Neri, D.F., and Rogers, W.H. The effects of color coding in geosit displays. I. Color as a redundant code. New London: Naval Submarine Medical Research Laboratory, Report Number 1061, September 1985.

Jameson, D. \& Hurvich, L.M. Dichromatic color language: "Reds" and "greens" don't look alike but their colors do. Sensory Processes, 1978 , 2. pp. 146-155.

Jerome, C.W. The rendering of ANSI safety colors. Journal of the Illuminating Engineering Society, 1977, April, p. 180-183.

Kelly, K.L. and Judd, D.B. Color: Universal Language and Dictionary of Names. Washington, D.C.: National Bureau of Standards, Special Publication 440, December 1976.

Lozano, R.D. The visibility, color and measuring requirements of road signs. Lighting Research and Technology, 1980, 12, pp. 206-212.

MacDonald, W.A. and Cole, B.L. Evaluating the role of color in a flight information cockpit display. Ergonomics, 1988, 31, pp. 13-37. 
MacNeil, R.F. Colors and legibility: Caution and warning data-plates . U.S. Army Technical Note 3-65. Aberdeen Proving Ground, Human Engineering Laboratories, Nov 1965.

Marr, D. Vision. San Francisco: W.H. Freeman \& Co., 1982.

Manual on Uniform Traffic Control Devices (MUTCD) Washington, DC. U.S. Department of Transportation, Federal Highway Administration. ANSI D6.1

- 1978. Revision No. 4, March 1986.

Mollon, J.D. On the origins of polymorphisms. In National Research Council, Committee on Vision, Frontiers of Visual Science: Proceedings of the 1985 Symposium. Washington, D.C. National Academy Press, 1987, pp. 160-168.

Nathan, J., Henry, G.H., and Cole, B.L. Recognition of colored road traffic light signals by normal and color-vision-defective observers. Journal of the Optical Society of America, 1964, 54, pp.1041-1045.

Olson, P.L. and Bernstein, A. The nighttime legibility of highway signs as a function of their luminance characteristics. Human Factors, 1979, 21, pp. $145-160$.

Olson, P.L. Minimum requirements for adequate nighttime conspicuity of highway signs. Ann Arbor: University of Michigan, UMTRI-88-8, 1988.

Piantanida, T.P. and Nathans, J. Molecular genetics of human color vision polymorphism. In National Research Council, Committee on Vision, Frontiers of Visual Science: Proceedings of the 1985 Symposium.

Washington, D.C.: National Academy Press, 1987, pp. 145-149.

Pointer, M.R. Color discrimination as a function of observer adaptation. Journal of the Optical Society of America, 1974, 64, pp. 750-759.

Pokorny, J., and Smith, V.S. The functional nature of polymorphism of human color vision. In National Research Council, Committee on Vision, Frontiers of Visual Science: Proceedings of the 1985 Symposium.

Washington, D.C. National Academy Press, 1987, pp. 150-159.

Pokorny, J. and Smith, V.C. Eye disease and color defects. Vision Research, $1986, \underline{26}$, pp. 1573-1584.

Rennilson, J.J. Chromaticity measurements of retroreflective material under nighttime geometry. Applied Optics, 1980, 19, pp. 1260-1267.

Robertson, A.R. The CIE 1976 color-difference formulae. Color Research and Application. 1977,2 , pp. 7-11.

Robinson, C.G. Color in traffic control. Traffic Engineering, 1967, May, pp. 25-29. 
Schiff, W. Perception: An Applied Approach. Boston: Houghton Mifflin, 1980 .

Shinar, D. The effects of expectancy, clothing reflectance, and detection criterion on nighttime pedestrian visibility. Human Factors, 1985,27 , pp. 327-333.

Sloan, I. L. and Habel, A. Recognition of red and green point sources by color-deficient observers. Journal of the Optical Society of America,

1955b, 45, pp. 599-601.

Sloan, L.L. and Habel, A. Color signal systems for the red-green color blind. An experimental test of the three-color system proposed by Judd. Journal of the Optical Society of America, 1955, 45, pp. 592-598.

Terstiege, H. Colors of transilluminated traffic signs. Lighting Research and Technology, 1980, 12, pp. 69-72.

Thornton, W.A. The design of safety-colors. Journal of the Illuminating Engineering Society, 1977,6 , 92-99.

Uchikawa, K. and Boynton, R.M. Categorical color perception of Japanese observers: Comparison with that of Americans. Vision Research, 1987, 27, Pp 1825-1833.

Uchikawa, K., Uchikawa, H., Kaiser, P.K. Equating colors for saturation and brightness: The relationship to luminance. Journal of the Optical Society of America. 1982, 72, pp. 1219-1231.

Verriest, G., Neubauer, O., Marre, M., and Uvijls, A. New investigations concerning the relationships between congenital color vision defects and road traffic security. International Ophthalmology, 1980, 2, Pp. 87-99. Referenced by Cole and Vingrys, 1985.

Vingrys, A.J. and Cole, B.L. Origins of Color Vision Standards Within the Transport Industry. Report to the Australian Department of Aviation, May 1986.

Vos, J.J., Lazet, A., and Bouman, M.A. Visual contrast thresholds in practical problems. Journal of the Optical Society of America, 1956, 46, pp. 1065-1068.

Werner, J.S. and Wooten, B.R. Opponent chromatic mechanisms: Relation to photopigments and hue naming. Journal of the Optical Society of America, $1979, \underline{69}$, p. 422-434.

Worthey, J.A. Opponent-colors approach to color rendering. Journal of the Optical Society of America, 1982, 72, Pp. 74-82.

Wright, W.D. The Measurement of Color, 4th Ed., New York: Van Nostrand Co, 1969 . 
Wright, W.D. Researches on Normal and Defective Color Vision. London: Henry Kimpton, 1946, pp. 209-255.

Wyszecki, G. and Stiles, W.S. Color Science: Concepts and Methods, Quantitative Data and Formulae. New York: Wiley, 1982.

\section{Other Color Specification Standards}

Association Francaise de Normalisation (AFNOR). Methodes et Appareils de Mesures des Caracteristiques Colorimetriques. X08-012, Dec 1974.

Australian Standard 1614. The Design and Use of Reflectorized Signs for Mines and Tunnels. AS 1614-1985.

Australian Standard 2700. Color Standards for General Purposes. AS 2700-1985.

Australian Standard 1318. SAA Industrial Safety Color Code. Use of Color for the Marking of Physical Hazards and the Identification of Certain Equipment in Industry. AS 1318-1985.

Australian Standard 1319. Safety Signs for the Occupational Environment. AS1319 - 1979.

South African Bureau of Standards. Standard Specification for RetroReflectors (Reflex Reflectors). SABS 513-1985. 


\section{A.1 Background}

\section{A.1.1 Feasibility of Retroreflective Color Specification}

The daytime color specification given in LS 300C (1977) specifies a test method using instruments with $45 / 0$ (or the reverse) illuminating and viewing geometry. The daylight sources are either diffuse (skylight) or directional (sunlight), and when directional can come from any direction. The viewing angle in daylight is roughly zero (perpendicular to the plane of the sign) but varies as the observer approaches the sign.

At night the viewing geometry is similar, but the illuminating geometry is quite different. For the minority of signs which have their own light sources, illumination is approximately at $45^{\circ 9}$. For the great majority of signs, the illumination is from approaching headlamps and is similar to the viewing geometry, given that the observer is almost directly behind one of the headlamps. However, at nighttime the peculiar optical characteristics of the retroreflective sheeting dominate the visibility situation, specifically the narrow observational angles and the larger and more rapidly changing entrance angles.

Can a practical nighttime color standard be written? If so, can a practical test method be written for both laboratory and field testing? A laboratory test method is needed for acceptance of new material. A field test method is needed for determining whether existing signs need replacement; it might also be used for accepting new material.

The procedures and equipment employed by Rennilson (1980) can be used to measure existing specimens known to be satisfactory for both daytime and nighttime use, and from these data a draft nighttime specification could be written. However, such equipment is much more expensive, and the laboratory space needed much more extensive, than that used now for testing for compliance with the daytime color specification. Only a minority of users now employ even this less expensive instrumentation.

If sufficient specimens of all the colors from all the manufacturers are measured in the laboratory, using a variety of observational and entrance angles, as was done by Rennilson (1980), one (or more) nighttime color specification can be written, along with appropriate test methods. It is to be expected that the above measurements will indicate differences in the results obtained on different types of sheeting -enclosed lens, encapsulated lens, prismatic, etc. -- and only extensive review of the data will determine the actual feasibility of such

${ }^{9}$ The contribution from vehicle headlamps to the overall illuminance on an overhead, externally illuminated sign remains to be specified. 
nighttime color specification(s).

One important factor not explored in this study is the differences between the new materials used as measurement specimens and the weathered materials which are candidates for replacement. Such a study will have to use both instrumental and visual test methods, as did the original work that resulted in the daytime color specification. One may find that optical differences that occur among different materials when new will be quite different for weathered retroreflectors.

A second important factor, noted but not extensively explored in the present study, relates to variations in the spectral reflectance characteristics of colors of the same name, both among different products of the same manufacturer and between manufacturers. Among the set of 20 specimens which were measured on the outdoor photometric range, it was noted that one of the three reds had a rather different curve from the other two; this was a difference between different manufacturers. The two browns, from the same manufacturer, have different curve shapes. These curve shape differentials may cause variations in the way the respective colors shift as a function of being viewed under different light sources.

It would seem that a goal for a practical specification and test method would be one that could be performed either in the laboratory or in the field, using the same instrumentation and dedicated light source. Such a test method, when used in the field, should be such that it can be performed with vehicle-mounted equipment by personnel with a minimum of training. The cost of instrumentation and light source can be limited to about $\$ 8,000$ so that it might be feasible for a spectrum of different government agencies.

\section{A2. Field Measurements}

The principal literature source on color measurement of retroreflectors under nighttime conditions is the paper by Rennilson (1980) in which he described the results of spectroradiometric measurements made under laboratory conditions which included four different observation angles and four different entrance angles, a total matrix of 16 different conditions. Rennilson's reference point was the $0.2^{\circ}$ observation angle and $-4^{\circ}$ entrance angle which is a standard geometry used in the evaluation of highway sign materials for intensity of retroreflected light. With this geometry as the zero point he computed chromaticity differences from it for the other fifteen geometric conditions.

Rennilson's study showed that for observation angles up to $0.5^{\circ}$ and entrance angles up to $15^{\circ}$ the chromaticity differences were minor. However, for the next incremental increase in angular deviation, to $1.0^{\circ}$ observation and $30^{\circ}$ entrance angles, chromaticity differences increased to an important degree.

The Manual on Uniform Traffic Control Devices (1978, rev. 1986) states that highway signs should have the same apparent color day and night. 
This is naive in view of abundant information to the contrary with respect to the appearance of colors under a variety of light sources (Collins et al., 1986). Color rendition of light sources has been explored in some detail in recent years, and much of this work has included the specific sources used in highway and highway sign illumination. The chromaticity shift with change in light source is most dramatic for more vivid colors, such as highway sign color. At the same time one can assume that drivers learn the appearance of familiar signs under various conditions of illumination, minimizing the negative aspects of these color shifts.

In an unpublished paper by Rennilson and Hale results were reported of viewing highway sign colors simultaneously under sunlight and CIE Illuminant A, typical of automotive headlamps. The color shifts were quite dramatic, and would have been more so had the sunlight been replaced by any other phase of daylight, which would have been more blue. As one example, highway blue under Illuminant $A$ is visually closer to highway green under sunlight, than it is to highway blue under sunlight.

As background for developing a field test method for color measurement of retroreflective materials, a Minolta CS 100 Luminance Meter was used on an outdoor photometric range to measure a series of 20 retroreflective specimens, consisting of 7 examples of high intensity materials and 13 engineering grade specimens.

As background, it was noted that a field test method should have several requirements:

1. Be capable of making measurements from a variety of distances, as opposed to addressing the sample directly, as is usual with colorimeters and spectrophotometers.

2. Be able to make measurements within an entrance angle spread of \pm $15^{\circ}$ primarily from a vehicle parked at the roadside, with the minimum necessity to dismount from the vehicle to approach the sign.

3. Include the use of a telespectroradiometer or telecolorimeter combined with a high intensity light source representative of CIE Illuminant A $(2856 \mathrm{~K})$.

To develop the field test method the outdoor photometric range used as a light source, an automobile headlamp at a range of $45 \mathrm{ft}(13.7 \mathrm{~m})$ and a specimen holder capable of providing entrance angles from 0 to $\pm 45^{\circ}$, with a tolerance of $\pm 1^{\circ}$. Light source color temperature was determined to be $2960^{-} \mathrm{K}$.

The 12 in by 12 in $(0.3 \mathrm{~m}$ by $0.3 \mathrm{~m})$ specimens were measured using a Minolta CS-100 telecolorimeter, with an acceptance aperture of $1^{\circ}$. It was deployed at a range so that the acceptance angle subtended a circle 8 in $(0.2 \mathrm{~m})$ in diameter, just under the accessible specimen width ( 8.5 in or $0.21 \mathrm{~m}$ ) at the maximum entrance angle. 
The CS100 telecolorimeter was positioned within the edge of the incident light beam so as to minimize the observation angle. Facilities were not available to accurately determine the observation angle, although it was designed to be as low as possible and was maintained throughout the measurements.

Selected results from the field measurements are shown in table A1. The measurements were made at entrance angles of $0,15,20,25$, and $30^{\circ}$, with the data being presented in that sequence. Table Al presents data for white, red; orange, yellow, blue, green, and brown engineering and high intensity grade samples. Data for blue, green, and brown samples are shown in CIELAB, while the data for the other colors are in CIE $x, y$ because their luminance was too high for the meter to convert the data into CIELAB. Little change with entrance angle is obvious for these colors. They are all quite close to the spectrum locus in the CIE 1931 chromaticity diagram. Each color shows a reduction in saturation as the entrance angle increases. For the 13 engineer grade colors, the same pattern of color change with entrance angle is apparent as for high intensity colors. This group includes two blue specimens which behaved similarly to brown and green.

The field data indicate that increasing entrance angle beyond $15-20^{\circ}$ creates scatter in the measurements for green, blue, and brown samples. It also confirms that accurate positioning of the light source close to the receptor is impossible to do with car headlamps and suggests the need for developing a precision-mounted light source for the telecolorimeter. Experience with the crude field method was used in writing the following test method for measuring highway signs. 
Table A1. Data obtained for retroreflective red, orange, yellow, blue, green, and brown samples under field conditions at night with entrance angles of $0,15,20,25$, and $30^{\circ}$.

Engineering Grade

$\begin{array}{clccc}\text { Angle } & \text { Sample } & \text { Y } & \text { x } & y \\ 0 & \text { Red 1 } & 127 & 0.6525 & 0.3419 \\ 15 & \text { Red 1 } & 116 & 0.6534 & 0.3419 \\ 20 & \text { Red 1 } & 109 & 0.6540 & 0.3414 \\ 25 & \text { Red 1 } & 101 & 0.6551 & 0.3405 \\ 30 & \text { Red 1 } & 093 & 0.6558 & 0.3400\end{array}$

\begin{tabular}{|c|c|c|c|c|}
\hline Angle & Sample & $Y$ & $\mathrm{x}$ & $y$ \\
\hline 0 & Orange 1 & 273 & 0.5984 & 0.3781 \\
\hline 15 & Orange 1 & 229 & 0.6080 & 0.3766 \\
\hline 20 & Orange 1 & 215 & 0.6091 & 0.3761 \\
\hline 25 & Orange 1 & 202 & 0.6098 & 0.3758 \\
\hline 30 & Orange 1 & 186 & 0.6104 & 0.3757 \\
\hline
\end{tabular}

$\begin{array}{ccrrr}\text { Angle } & \text { Sample } & \text { Y } & \text { x } & y \\ 0 & \text { Yellow 1 } & 415 & 0.5605 & 0.4330 \\ 15 & \text { Yellow 1 } & 363 & 0.5622 & 0.4331 \\ 20 & \text { Yellow 1 } & 339 & 0.5628 & 0.4308 \\ 25 & \text { Yellow 1 } & 314 & 0.5632 & 0.4303 \\ 30 & \text { Yellow 1 } & 290 & 0.5635 & 0.4300\end{array}$

$\begin{array}{clcc}\text { Angle } & \text { Sample } & \text { L* } & \text { a* } \\ 0 & \text { Green 1 } & 95.75 & -140.76 \\ 15 & \text { Green 1 } & 93.05 & -138.23 \\ 20 & \text { Green 1 } & 91.47 & -136.54 \\ 25 & \text { Green 1 } & 88.44 & -133.13 \\ 30 & \text { Green 1 } & 86.30 & -130.79\end{array}$

$\begin{array}{ccccc}\text { Angle } & \text { Sample } & \text { L* } & \text { a* } & \text { b* } \\ 0 & \text { Blue 1 } & 60.71 & -63.36 & -36.40 \\ 15 & \text { Blue 1 } & 56.93 & -59.98 & -36.20 \\ 20 & \text { Blue 1 } & 54.10 & -56.73 & -35.93 \\ 25 & \text { Blue 1 } & 52.46 & -54.49 & -35.70 \\ 30 & \text { Blue 1 } & 50.57 & -52.18 & -35.41\end{array}$

$\begin{array}{clccc}\text { Angle } & \text { Sample } & \text { L* } & \text { a* } & \text { b* } \\ 0 & \text { Brown 1 } & 32.59 & 18.40 & 29.60 \\ 15 & \text { Brown 1 } & 32.39 & 17.94 & 29.57 \\ 20 & \text { Brown 1 } & 30.75 & 17.70 & 29.71 \\ 25 & \text { Brown 1 } & 31.85 & 16.04 & 27.80 \\ 30 & \text { Brown 1 } & 31.11 & 16.62 & 26.30\end{array}$

$\mathrm{b} *$

65.88

65.39

64.39

63.03

61.87

b*

$-36.40$

$-35.93$

$-35.70$

$-41$

*

9.57

9.71

26.30

\section{High Intensity}

Sample
Red 2
Red 2
Red 2
Red 2
Red 2

Sample

Orange 2

Orange 2

Orange 2

Orange 2

Orange 2

$\mathrm{Y}$
97.8
96.9
96.7
96.7
210.0

$\mathrm{x}$

0.6800

0.6812

0.6818

0.6824

0.6780

$Y$
369
377
378
372
368

$x$
0.6258
0.6262
0.6257
0.6254
0.6253

$y$
0.3706
0.3706
0.3712
0.3711
0.3717

Sample

Yellow 2

Yellow 2

Yellow 2

Yellow 2

Yellow 2

$\begin{array}{cc}x & y \\ 0.5737 & 0.4257 \\ 0.5740 & 0.4256 \\ 0.5737 & 0.4259 \\ 0.5737 & 0.4260 \\ 0.5741 & 0.4257\end{array}$

0.3168

0.3159

0.3155

0.3149

0.3200
Sample

Green 2

Green 2

Green 2

Green 2

Green 2

Sample

Blue 2

Blue 2

Blue 2

Blue 2

Blue 2

Sample

Brown 2

Brown 2

Brown 2

Brown 2

Brown 2

L*
114.53
114.04
113.61
112.01
110.37
L*
27.84
28.45
28.45
29.26
29.66

L* 33.58

36.19

33.43

33.15

33.77 a*

$-164.29$

$-165.15$

$-165.46$

$-164.43$

$-163.11$ 
A3. Test Method for Color Measurement of Retroreflective Highway Signs Under Nighttime Illumination

\section{A3.1. Introduction}

Highway signs are produced in a variety of colors as specified by the Federal Highway Administration. Safety considerations dictate that such specifications contain minimum requirements. In order to determine if new and existing signs meet such requirements, it is necessary to provide a standard test method.

\section{A3.2 Scope}

A3.2.1 This test method is applicable to all types of retroreflective materials approved for highway sign usage. Its purpose is to permit the user to determine when environmental conditions, damage or other factors have rendered the sign unsafe for its intended purpose, as well as to evaluate new materials.

\section{A3.3 Applicable Documents}

A3.3.1 Manual on Uniform Traffic Control Devices (MUTCD), Revision No. 4 March 1986.

A3.3.2 ASTM Standards ${ }^{10}$.

A3.3.2.1 E284 Standard Definitions of Terms Relating to Appearance of Materials.

A3.3.2.2 E808 Standard Practice for Describing Retroreflection

A3.3.2.3 E811 Standard Practice for Measuring Colorimetric Characteristics of Retroreflectors under Nighttime Conditions.

A3.4. Terminology

$\mathrm{A} 3.4 .1$ See A3.3.2.1, A3.3.2.2 and A3.3.2.3 above

A3.5. Summary of Test Method

A3.5.1 Color Measurements are made with a telecolorimeter having a $1^{\circ}$ acceptance angle, at a variety of ranges so that the work can be accomplished with maximum speed and safety, during hours of darkness.

A3.5.2 With the telecolorimeter is a high intensity light source representing CIE Illuminant $A$, with a color temperature of $2856 \pm 25 \mathrm{~K}$, providing an observation angle of $0.33^{\circ}$ as currently specified by the CIE.

${ }^{10}$ ASTM standards are available from ASTM, 1903 Race St., Philadelphia, PA 19003. 
A3.5.3 Measurements shall be made so that the entrance angle does not exceed $15^{\circ}$. (Further research is needed to confirm that there is minimal scatter in chromaticity measurements up to entrance angles of $15^{\circ}$ ).

A3.5.4 The beam spread of the light source shall be such that at the minimum range of $3 \mathrm{~m}$ ( $9.8 \mathrm{ft}$ ), it covers a $127 \mathrm{~mm}$ ( $5 \mathrm{in}$ )

diameter circle, which will increase in diameter as the range increases. This permits the color of highway signs which are ordinarily small (Stop, Curve, Warning, Speed Limit, etc.) to be measured at close range while excluding the legend from the measurement area. The procedure will also permit those signs (directional guide signs, route markers, etc.) which are usually large and often somewhat inaccessible to be measured at a greater range. The intent here is to measure the various types of signs at the ranges at which they are readily accessible, and at which the instrument's acceptance angle will subtend an uninterrupted circle of a size that will be available on that type of sign.

A3.5.5 Procedures for measuring specular gloss are given in ASTM D523. It is not appropriate to measure gloss as part of this test method.

A3.6. Significance and Use

A3.6.1 Highway signs are color coded, with the background color indicating the sign's function, such as regulatory, warning and guidance to locations or intersecting roads, and location of off-highway facilities such as fuel and food. In order for the signs to be differentiated from a variety of backgrounds, and then identified as to color, and therefore function, it is necessary that their color remain sufficiently vivid (saturated) as to be conspicuous.

A3.6.2 Specifications for highway sign materials include standard LS-300C which provides a specification for the color in daylight, along with a test method. This document provides a test method for determining color at night, and can be used to determine when the sign must be replaced because it no longer meets minimum standards for conspicuity during hours of darkness.

\section{A3.7. Apparatus}

A3.7.1 Telecolorimeter. This is a tristimulus colorimeter capable of measuring reflecting materials and self-luminous material at ranges from $3.5 \mathrm{~m}(11.5 \mathrm{ft})$ to infinity. It must have acceptance angles between $0.5^{\circ}$ and $1.5^{\circ}$ in order to measure sign specimens of the appropriate size over the range of interest. 
A3.7.2 Light Source. The light source shall be tungsten and shall have a color temperature of $2856 \pm 25 \mathrm{~K}$, equivalent to CIE Illuminant A. It shall have intensity and beam spread characteristics appropriate to the task.

A3.7.3 The observation angle between light source and telecolorimeter shall be $0.333^{\circ 11}$. The light source may be built into the telecolorimeter or attached to it by mechanical means, or subject to any other arrangement so long as all of the above requirements are met.

\section{A3.8. Test Specimens}

Signs are to be measured in place and are not to be cleaned or otherwise prepared for examination. The instrument-light source combination is meant to be brought to the sign in such a manner that it addresses the sign within the permissible range and entrance angle.

\section{A3.9. Calibration and Standardization}

A3.9.1 The light source shall be calibrated to meet the requirements of section A3.7.2. (See Wyszecki and Stiles, 1982). The calibration should be checked prior to each use of the apparatus. Note that the telecolorimeter, with a suitable dedicated computer, can be used for testing the light source.

A3.9.2 The telecolorimeter shall be calibrated in the following manner. Select the local reference standard of the same nominal color as the specimen to be measured. Activate light source-instrument combination and follow instrument manufacturer's instructions for calibration with local color standard. The reference standard has been calibrated spectroradiometrically for a $0.33^{\circ}$ observation angle and $0^{\circ}$ entrance angle. These geometric conditions should be set with respect to instrument and specimen.

A3.9.3 Reference standards for telecolorimeter calibration are available as noted below. 12

${ }^{11}$ The $0.33^{\circ}$ observation angle in $\mathrm{A3} .7 .3$ was selected to conform with the document being written by CIE Committee 2-9 for nighttime measurement of these materials by spectroradiometry.

${ }^{12}$ Calibrated reference standards are available from Hale Color Consultants, Inc., 1505 Phoenix Rd., Phoenix, MD 21131. 
A3.10. Report will include:

A3.10.1 Name, serial number and manufacturer of telecolorimeter and light source.

A3.10.2 Source and calibration information for calibration standards.

A3.10.3 CIE $Y, x, y$ and CIELAB $L^{*}, a^{*}, b^{*}$ data for CIE Illuminant $A$, for each specimen.

A3.10.4 Measurement range (estimated).

A3.11. Precision and Bias

A3.11.1 Precision and bias data are being developed for this test method. 
A4.1 Future Research on Use of Degraded Signs and Evaluation of Day-Night Color and Gloss

A4.1.1 Degraded Signs

If highway signs were made from paint or similar materials, with conventional reflection properties, there is little question that a good correlation could be found between day and night color, thus permitting the measurements to be made during daylight. Daylight measurements can be made more quickly, under safer conditions, and at lower costs.

It is the singular geometric reflection properties of retroreflective materials that cause the important differences between both their light reflection characteristics and their color between daytime and nighttime. And it is the degradation with age and weathering of such properties that one would wish to evaluate. Thus even if a useful correlation could be determined between day and night appearance of new materials, such as those used in the current study, there is no guarantee that this correlation would be true for the very type of degraded material that should be evaluated. While there is merit in being able to measure the color of retroreflective material for both day and night appearance, it is probable that the more important factor for evaluation is the appearance of this same material when it has deteriorated near the point where it may be necessary to replace it. This should be the subject of a separate study. Some of the techniques employed in the present study will be useful in such further research.

\section{A4.1.2 Gloss Specification for Retroreflective Signs}

Current specifications for evaluation of gloss specify the use of ASTM D 523 Standard Test Method for Specular Gloss, using $85^{\circ}$ geometry.

However, this method includes three separate geometries, 20,60 and $85^{\circ}$, and specifies that $60^{\circ}$ geometry is to be used for most specimens. It continues to specify that if $60^{\circ}$ gloss is higher than 70 units, then the $20^{\circ}$ geometry is to be employed. Similarly, D 523 notes that when $60^{\circ}$ gloss is less than 10 units, $85^{\circ}$ geometry should be used.

It has long been known that none of these geometries are linear with visual gloss appraisal. Specifically, the $60^{\circ}$ geometry scale is compressed at both the high and low gloss ends, so that large visual gloss differences measure as very little different; on the other hand, the $60^{\circ}$ geometry is usually quite adequate between 10 and 70 gloss units. Similarly, the 20 and $85^{\circ}$ geometry have an expanded scale in the high and low gloss regions, respectively, and compressed scales in other regions.

The literature search has disclosed no references to explain the use of $85^{\circ}$ geometry for high gloss retroreflective sheeting, when $D 523$

specifies $20^{\circ}$ gloss for such specimens. However, a leading researcher at one of the manufacturers suggests that $20^{\circ}$ geometry has been tried, and rejected because at this angle some of the incident light penetrates the first surface and is retroreflected within the acceptance cone of the 
gloss measuring instrument, adding to the specular beam and resulting in a false reading.

Inasmuch as the $85^{\circ}$ gloss scale is importantly compressed for high gloss specimens, one must be careful in setting minimum values for new material. A study is indicated where all current materials are employed, as different types of first surface plastic sheeting are employed within the industry. Consideration should be given to $60^{\circ}$ and $85^{\circ}$ geometry, as well as other geometries. It might also be useful to consider other types of gloss evaluation, such as distinctness of image gloss as well as to reappraise the requirement for a gloss specification.

While new materials have a high gloss finish, outdoor aging results in deterioration of both gloss and color. For new materials the 45/0 geometry specified for color measurement probably excludes the specular beam completely or almost so, permitting color and gloss to be separately measured. It is also quite possible that a sphere instrument with a light trap for excluding the specular component would work as well for the color measurement of new material. The 45/0 geometry is that which has historically been used in tristimulus colorimeters, the usual instrument employed with retroreflective material, and the wide availability of these instruments (which have a lower cost than a spectrophotometer) is a good reason for specifying this geometry.

As retroreflective sheeting deteriorates under environmental conditions, the high gloss surface gradually assumes a lower gloss. At some point in this degradation, the specularly reflected beam may encompass such a wide angle that some portion of it falls within the acceptance cone for color measurement. It will then interfere with the color measurement, adding unmodified reflected light, amount unknown, to the chromatic characteristics of the specimen.

It thus appears that the entire matter of gloss behavior and its evaluation should be the subject of further study. It needs to be positively determined if it is necessary to evaluate gloss of both new and old materials; and if so, how this should be done.

A4.1.3 Future Research on Nighttime Color Measurement Using a Telecolorimeter

While it has been determined that the telecolorimeter used in'this experiment, the Minolta CS-100, is quite capable of making accurate and repeatable colorimetric measurement of retroreflective materials, it was not possible to calibrate it satisfactorily for measurements made at the range of entrance angles necessary for evaluation of highway signs at night.

It would be necessary to make extensive measurements by spectroradiometry of the full range of retroreflective materials, at a variety of entrance angles up to $30^{\circ}$, to select a series of calibration standards against which the instrument would be calibrated. It is likely that such a set of reference standards would be samples of retroreflective sheeting 
of reference standards would be samples of retroreflective sheeting previously calibrated on a spectroradiometer.

If the above experimental work determined that the telecolorimeter response did not change importantly over an entrance angle range from 0 to 15 or $20^{\circ}$, it would then seem possible that a standard and test method could be successfully written and employed for nighttime color measurement in the field. The range of entrance angles must be wide enough that field measurements could be confined to this range by a visual determination, and without reducing personnel safety or increasing time and difficulty of field measurement.

The CS-100 is currently calibrated using a white porcelain panel, for $45 / 0$ geometry. However, as $\mathrm{E} \mathrm{XXXX}^{13}$ specifies that colorimetric measurement of retroreflective sheeting should employ chromatic calibration standards which are themselves retroreflective, it is believed this approach would produce more accurate results.

${ }^{13} \mathrm{E}$ XXXX is the new ASTM E-12 Committee draft standard on Photoelectric Tristimulus Measurement of Object Colors, currently up for ASTM society ballot after approval by Committee E-12. It is referenced in the new draft specification for daytime measurement of highway sign material recently approved by task group of ASTM Committee D04.38 on Traffic Control Devices, which is intended to replace LS-300c. 
Would minor shifts in the highway color specifications enhance or degrade the observer's ability to discriminate these colors, or to identify them correctly under various light sources? Collins et al. (1986) showed that some sources tend to obscure the hue differences between colors adjacent to each other in color space, in the sequence red, orange, yellow, green, blue. However, while the first three of these hues are rather close to each other, the last two are more distant from the initial three, and from each other. This visual spacing as specified under daylight (CIE Illuminant C) directly relates to their visual spacing under other sources.

The original highway colors, red, yellow, green, blue and white, were formally specified some thirty years ago. The hue spacing of the four chromatic colors was certainly adequate, as was the contrast of these background colors with white and black used for legends. Red, green and blue were quite dark and contrasted well with white. Yellow, a light color, contrasted well with black.

When the FHWA recognized the need for additional sign colors, it proposed a vivid orange for highway construction sites, and a brown for parks, historic sites and similar locations. About this same time a purple color was tested in several locations; it was soon apparent that pigments available for purple color in signs did not have the requisite outdoor ageing characteristics needed for this application.

While pigment characteristics were not a problem for orange and brown, color spacing was. Both of these had to be placed in the available space between red and yellow. Orange was located midway between these two colors, and appropriate tolerances set to define the "color boxes" in CIE $\mathrm{x}, \mathrm{y}$ space; color tolerance charts were designed to provide visual interpretation of these tolerances, as had been done for the oxiginal colors. The same applied to brown, which was specified sufficiently dark so as not be confused with orange which occupies the same hue region.

A further problem with orange is that in vivid form it falls closer to the center of the lightness range between black and white than did any of the original four chromatic colors. Because contrast and legibility are largely a function of lightness contrast, less contrast is achievable between legend and surround with orange than with the other colors.

If color boundaries are shifted slightly to improve hue spacing, are pigments available with the necessary aging characteristics, especially in the more critical red, orange, yellow hue region? Manufacturers of retroreflective sheeting are understandably reticent to disclose proprietary data, or the results of their research with pigments necessary to produce other colors. However, any color shifts proposed would certainly be of modest magnitude, and time for necessary research and testing could be allowed. Improvements in correlation between accelerated testing and real-time aging serve to shrink the time-frame for such work. Where colors are now the product of two chromatic 
pigments, the hue shift proposed may be so minor that these same pigments could still be used, but in different ratios. Manufacture of highway sign materials is now highly competitive; this will be an incentive for rapid response to proposed new colors.

1. Table A2 contains Munsell specifications for the daylight color of both the ANSI and FHWA colors. The recommendations for switching to the ANSI specifications are partly based upon the data obtained by Collins et al. and the author's extensive experience in this field. Although it is desirable that they be subjected to suitable experimental investigation under retroreflective viewing conditions, the following points are pertinent.

A. The table below shows, in Munsell hue steps, the spacing between adjacent hues in the existing FHWA and ANSI Z53.1 standards :

\begin{tabular}{lll} 
Adjacent Colors & FHWA & $\underline{Z 53.1}$ \\
\hline Red to Orange & 3.25 & 5.25 \\
Orange to Yellow & 4.75 & 7.25 \\
Yellow to Green & 23.0 & 18.5 \\
Green to Blue & 19.5 & 19.5 \\
Blue to Red & 31.0 & 32.0
\end{tabular}

B. To increase the separation between Red, Orange and Yellow:

1. Consider changing Highway orange to the Z53.1 orange, increasing the red-orange hue spacing from 3.25 steps to 5.75 steps.

2. Also consider switching Highway yellow to the Z53.1 yellow. This would increase the orange-yellow hue spacing from 4.75 steps to 7.25 steps.

3. Consider the possibility of further research to determine if colors which are intermediate between the ANSI and highway specifications might be recognized more accurately. Such colors were not explicitly evaluated by Collins et al., although their data suggest that several other blue, green, and yellow colors could be further evaluated for highway applications.

C. No color change has been suggested for White or Brown. 
Table A2. Munsell notation for FHWA and ANSI Highway Color Centroids and Tolerances.

$\underline{\text { Color }}$

RED

Centroid

$\mathrm{H} \pm$
$\mathrm{V} \pm$
$\mathrm{C} \pm$

ORANGE

Centroid

$\mathrm{H} \pm$
$\mathrm{V} \pm$
$\mathrm{C} \pm$

BROWN

Centroid

$H \pm$
$V \pm$
$C \pm$

YELLOW

Centroid

$\mathrm{H} \pm$

V \pm

GREEN

Centroid

$\mathrm{H} \pm$
$\mathrm{V} \pm$
$\mathrm{C} \pm$

BLUE

Centroid

$\mathrm{H} \pm$

$\mathrm{V} \pm$
FHWA

$$
\begin{aligned}
& 7.0 \mathrm{R}-3.5 / 13 \\
& 6.0-8.0 \mathrm{R} \\
& 3.0-4.0 \\
& 12.0-15.0
\end{aligned}
$$

2.5YR $\quad 5.5 / 14$

$1.25-3.75 \mathrm{YR}$

$5.0-6.0$

$12.0-15.0$

5. OYR $2.75 / 5$

$2.5-7.0 \mathrm{YR}$

$2.25-3.25$

$4.0-6.0$

$\begin{array}{rlrl}10 . \mathrm{YR} & 7.5 / 14 & 5.0 Y & 8.0 / 12 \\ 8.5 \mathrm{YR} & -2.0 \mathrm{Y} & 3.5 & =6.54 \mathrm{Y} \\ 6.5 & -8.0 & 7.5-8.5 \\ 12.0 & -16.0 & 10.0-14.0\end{array}$

$7.5 \mathrm{G} \quad 3.0 / 8$

$5.0 \mathrm{G}-0.5 \mathrm{BG}$

$2.25-3.75$

$6.0-10.0$

2. $5 \mathrm{~PB} 3 / 8$

$10 B-5 P B$

$2.0-3.5$

$6.0-10.0$
ANSI

7. $5 \mathrm{R} 4 / 14$

$6.5-8.5 R$

$3.5-4.5$

$12.0-16.0$

$$
5.0 \mathrm{YR} \quad 6.0 / 15
$$

$3.75-6.25 \mathrm{YR}$

$13.0-16.0$

$5.0 Y R \quad 2.75 / 5$

$2.5-7.0 \mathrm{YR}$

$2.25-3.25$

$4.0-6.0$
$5.5-6.5$ 
D. Changes in Lightness. Changes of this type impact chiefly on the contrast of the sign surrounds with the white or black legends. Recommendations are:

1. Red should be changed from Munsell 3.5 to 4.0 value, to agree with the lightness range of the 253.1 Red. Tolerances would be 3.5 to 4.5 .

2. Orange, if changed to the $\mathrm{Z53.1}$ orange, would then have a lightness of 6.0 value, instead of $5.5 /$, which will improve its contrast with its black legend. Where orange is contrasted with white, as in construction barricades, consideration should be given to retaining a lightness of 5.5 .

3. Yellow should increase in lightness from 7.5 to 8.0 , to agree with the $\mathrm{Z53} .1$ Yellow. The existing yellow lightness minimum is at 6.5 value, which is quite dark. A lightness range with a 7.0 value minimum would seem more suitable. No light limit specification is necessary or desirable.

4. Green and blue have very dark lightness minima, as well as dark specifications for their centroid colors. Both colors should have their centroids lightened by 0.5 value steps, at 3.5 , with light and dark tolerances at 4.0 and 3.0 .

E. Changes in Saturation. If saturation increases are feasible for retroreflective materials, experiments should be performed to determine if such increases would improve visibility and legibility.

2. Appendix B shows CIELAB data for the existing FHWA colors in tabular form for the corner points of the color boxes, and the range of lightness, in CIELAB L*, $a *, b *$ form, for the CIE $19762^{\circ}$ Observer with data computed for CIE Illuminant $C$. The table is difficult to use, however, because extensive interpolation is needed between the light and dark levels for the corner boxes in CIELAB. Further work is needed to define usable color boxes in CIELAB. Although ASTM D3134 provides CIELAB tolerances, it is in the process of being revised so that it is premature to express tolerances in D-3134 format until such tolerances have been determined to be firmer. 
Appendix B. CIE $x, y$ and CIELAB specifications for Highway Colors

\begin{tabular}{|c|c|c|c|c|c|c|}
\hline Color & $\mathrm{x}$ & $\mathrm{y}$ & $Y$ & L* & $a *$ & $b *$ \\
\hline White 1 & 0.305 & 0.290 & 35 & 65.75 & 8.31 & -8.05 \\
\hline White 2 & 0.350 & 0.342 & 35 & 65.75 & 5.04 & 12.22 \\
\hline White 3 & 0.321 & 0.361 & 35 & 65.75 & -11.32 & 13.17 \\
\hline White 4 & 0.276 & 0.308 & 35 & 65.75 & $-10 \cdot 44$ & -6.40 \\
\hline Red 1 Dark & 0.602 & 0.317 & 8 & 33.98 & 53.09 & 34.46 \\
\hline Red 1 Light & 0.602 & 0.317 & 12 & 41.22 & 60.77 & 39.45 \\
\hline Red 2 Dark & 0.664 & 0.336 & 8 & 33.98 & 56.68 & 58.59 \\
\hline Red 2 Light & 0.664 & 0.336 & 12 & 41.22 & 64.88 & 71.06 \\
\hline Red 3 Dark & 0.644 & 0.356 & 8 & 33.98 & 48.78 & 58.59 \\
\hline Red 3 Light & 0.644 & 0.356 & 12 & 41.22 & 55.84 & 71.06 \\
\hline Red 4 Dark & 0.575 & 0.356 & 8 & 33.98 & 38.98 & 39.01 \\
\hline Red 4 Light & 0.575 & 0.356 & 12 & 41.22 & 44.62 & 44.66 \\
\hline Orange 1 Dark & 0.535 & 0.375 & 18 & 49.50 & 37.57 & 46.56 \\
\hline Orange 1 Light & 0.535 & 0.375 & 30 & 61.65 & 44.54 & 55.20 \\
\hline Orange 2 Dark & 0.607 & 0.393 & 18 & 49.50 & 46.15 & 85.34 \\
\hline Orange 2 Light & 0.607 & 0.393 & 30 & 61.65 & 54.71 & 106.30 \\
\hline Orange 3 Dark & 0.582 & 0.417 & 18 & 49.50 & 35.24 & 84.77 \\
\hline Orange 3 Light & 0.582 & 0.417 & 30 & 61.65 & 41.78 & 105.35 \\
\hline Orange 4 Dark & 0.535 & 0.399 & 18 & 49.50 & 31.02 & 54.30 \\
\hline Orange 4 Light & 0.535 & 0.399 & 30 & 61.65 & 36.78 & 64.38 \\
\hline Brown 1 Dark & 0.445 & 0.353 & 4 & 23.67 & 14.93 & 14.70 \\
\hline Brown 1 Light & 0.445 & 0.353 & 9 & 35.98 & 19.56 & 19.26 \\
\hline Brown 2 Dark & 0.604 & 0.396 & 4 & 23.67 & 27.12 & 40.81 \\
\hline Brown 2 Light & 0.604 & 0.396 & 9 & 35.98 & 35.54 & 62.04 \\
\hline Brown 3 Dark & 0.556 & 0.443 & 4 & 23.67 & 14.65 & 40.69 \\
\hline Brown 3 Light & 0.556 & 0.443 & 9 & 35.98 & 19.20 & 61.77 \\
\hline Brown 4 Dark & 0.445 & 0.386 & 4 & 23.67 & 9.47 & 19.28 \\
\hline Brown 4 Light & 0.445 & 0.386 & 9 & 35.98 & 12.41 & 25.26 \\
\hline Yellow 1 Dark & 0.482 & 0.450 & 29 & 60.78 & 9.87 & 65.70 \\
\hline Yellow 1 Light & 0.482 & 0.450 & 45 & 72.89 & 11.43 & 76.06 \\
\hline Yellow 2 Dark & 0.532 & 0.465 & 29 & 60.78 & 17.44 & 102.33 \\
\hline Yellow 2 Light & 0.532 & 0.465 & 45 & 72.89 & 20.19 & 121.85 \\
\hline Yellow 3 Dark & 0.505 & 0.494 & 29 & 60.78 & 4.61 & 104.02 \\
\hline Yellow 3 Light & 0.505 & 0.494 & 45 & 72.89 & 5.34 & 124.48 \\
\hline Yellow 4 Dark & 0.475 & 0.485 & 29 & 60.78 & -0.04 & 77.86 \\
\hline Yellow 4 Light & 0.475 & 0.485 & 45 & 72.89 & -0.04 & 90.14 \\
\hline Green 1 Dark & 0.130 & 0.369 & 3.5 & 21.94 & -47.29 & -3.09 \\
\hline Green 1 Light & 0.130 & 0.369 & 9 & 35.98 & -64.78 & -4.23 \\
\hline Green 2 Dark & 0.180 & 0.391 & 3.5 & 21.94 & -36.44 & 1.61 \\
\hline Green 2 Light & 0.180 & 0.391 & 9 & 35.98 & -49.93 & 2.20 \\
\hline Green 3 Dark & 0.155 & 0.460 & 3.5 & 21.94 & -49.00 & 7.11 \\
\hline Green 3 Light & 0.155 & 0.460 & 9 & 35.98 & -67.13 & 9.75 \\
\hline Green 4 Dark & 0.107 & 0.439 & 3.5 & 21.94 & -60.72 & 2.85 \\
\hline Green 4 Light & 0.107 & 0.439 & 9 & 35.98 & -83.19 & 3.91 \\
\hline Blue 1 Dark & 0.147 & 0.075 & 1 & 8.99 & 27.97 & -45.78 \\
\hline Blue 1 Light & 0.147 & 0.075 & 4 & 23.67 & 44.39 & -72.68 \\
\hline Blue 2 Dark & 0.176 & 0.091 & 1 & 8.99 & 27.37 & -38.60 \\
\hline Blue 2 Light & 0.176 & 0.091 & 4 & 23.67 & 43.44 & -61.27 \\
\hline Blue 3 Dark & 0.176 & 0.151 & 1. & 8.99 & 6.38 & -23.97 \\
\hline Blue 3 Light & 0.176 & 0.151 & 4 & 23.67 & 10.13 & -38.05 \\
\hline Blue 4 Dark & 0.106 & 0.113 & 1 & 8.99 & -1.58 & -34.53 \\
\hline Blue 4 Light & 0.106 & 0.113 & 4 & 23.67 & -2.52 & -54.82 \\
\hline
\end{tabular}




\section{Appendix C. Definitions}

Definitions have been adopted from ASTM E284 as reprinted in ASTM Standards on Color and Appearance Measurement, 2nd Ed, 1987, except where noted.

Chromaticity - the color quality of a color stimulus definable by its chromaticity coordinates, or by its dominant (or complimentary) wavelength and its purity taken together. (CIE, 1983)

Chromaticity coordinates - the ratios of each of the tristimulus values of a color to the sum of the tristimulus values. (Chromaticity coordinates in the CIE system of color specification are designated by $\mathrm{x}, \mathrm{y}$, and $\mathrm{z}$ and by $\mathrm{a} * \mathrm{~b} *$ and $L *$ in the CIELAB system.)

Color - (1) (Perceived) attribute of visual perception that can be described by color names such as white, gray, black, yellow, brown, vivid red, deep reddish purple, or by combinations of such names.

Note 1 - Perceived color depends greatly on the spectral power distribution of the color stimulus, but also on the size, shape, structure, and surround of the stimulus area, the state of adaptation of the observer's visual system, and the observer's experience with similar observations.

(2) (psychophysical) characteristics of a color stimulus (that is, light producing a sensation of color) denoted by a colorimetric specification with three values, such as tristimulus values.

Note 1 - Tristimulus values are sometimes derived on a relative rather than an absolute basis. In this case they may need to be supplemented by the value of a suitable absolute photometric quantity.

Note 2 - The appearance of colors depends not only on their absolute tristimulus values, but also on the conditions under which they are viewed, including the nature of the surround; however, colors having the same absolute tristimulus values appear the same in identical viewing conditions. Spectrally different color stimuli can have the same absolute tristimulus values.

Color Difference - (1) Color difference (perceived), the magnitude and character of the difference between two colors described in such terms as redder, bluer, lighter, darker, grayer, or cleaner.

(2) Color difference (computed), - the magnitude and direction of the difference between two psychophysical color stimuli and their components computed from tristimulus values, or chromaticity coordinates and luminance factor, by means of a specified set of color-difference equations.

Colorimeter, tristimulus - Instrument that measures color, in terms of tristimulus values, often by the use of filters to convert the relative spectral power distribution of the illuminating source to that of a standard illuminant, and to convert the relative spectral sensitivity of the sensor to sensitivities prescribed by a standard observer.

Colorimetry - The science of color measurement.

Color space - A geometric space, usually of three dimensions in which colors are arranged systematically. 
Color temperature - of a source, the temperature usually expressed in kelvins, of a full radiator that would emit light of the same chromaticity as the source.

Contrast - (objective) - the degree of dissimilarity of a measured quantity such as luminance of two areas, expressed as number computed by a specified formula such as $\mathrm{C} \doteq \mathrm{L}_{2}-\mathrm{L}_{1} / \mathrm{L}_{1}$.

Footcandle ( $f c$ ) - The unit of illuminance when the foot is taken as the unit of length. It is the illuminance on a surface one square foot in area on which there is a uniformly distributed flux of one lumen, or the illuminance produced on a surface all points of which are at a distance of one foot from a directionally uniform point source of one candela. (IESNA, 1984)

Goniophotometer - Instrument that measures luminous flux as a function of angles of incidence or propagation.

Hue - The attribute of color perception by means of which a color is judged to be red, orange, yellow, green, blue, purple, or intermediate between adjacent pairs of these considered in a closed ring (red purple being an adjacent pair).

\section{Illuminance - Luminous flux incident per unit of area.}

Lightness - (1) the attribute of color perception by which a non-selfluminous body is judged to reflect more or less light. (2) The attribute by which a perceived color is judged to be equivalent to one of a series of grays ranging from black to white.

Luminance - Luminous flux in a beam, emanating from a surface, or falling on a surface, in a given direction, per unit of projected area of the surface as viewed from that direction, per unit solid angle.

Photometer - an instrument for measuring light.

Retroreflection - Reflection characterized by the flux in an incident beam being returned in directions close to the direction from which it came, this effect occurring over a wide range of incident angles.

Saturation - Attribute of a visual sensation according to which an area appears to exhibit more or less chromatic color, judged in proportion to its lightness or brightness.

Transilluminated signs - intended to be viewed by both daylight and by supplementary artificial illumination. "The materials used for the surfaces of transilluminated signs exhibit properties partially of transmission and partially of diffuse reflection. By day they are commonly seen by reflected daylight; but they may also be illuminated artificially by transmitted light, so as to make them visible by night or to enhance their visibility under poor daylight conditions. The color perceived is dependent on the color of the illuminant under the conditions of observation. In addition, particularly in the case of internally colored materials, there may be difference in color depending on whether the light is transmitted or reflected" (CIE, 1983, p23). 
NBS.114A (REV. 2.8C)

U.S. DEPT. OF COMM.

BIBLIOGRAPHIC DATA

SHEET (See in structions)

1. PUBLICATION OR REPORTNO.

NISTIR. 88-3894
2. Performing Organ. Report Nof 3. Publication Date

NOVEMBER 1988

4. TITLE ANO SUBTITLE

Evaluation of Colors for Use on Traffic Control Devices

5. $A \cup T H O R(S)$

6. PERFORMING ORGANIZATION (If joint or other than NBS, see in structions)

7. Contraed Grant No.

NATIONAL BUREAU OF STANDARDS

U.S. DEPARTMENT OF COMMERCE GAITHERSBURG, MD 20899

9. SPONSORING ORGANIZATION NAME AND COMPLETE ADDRESS (Street, City, State, ZIP)

Federal Highway Administration

6300 Georgetown Pike

McLean, VA 22101

10. SUPPLEMENTARY NOTES

Document describes a computer program; SF-185, FIPS Software Summary, is attached.

11. ABSTRACT (A 200-word or less factual summary of most significant information. If document includes a significant bibliogrophy or literoture survey. mention it here)

Color has traditionally been used to code safety information because of its ability to attract attention and evoke a rapid response. In the present report, research on color coding, highway safety color codes, color deficiency, retroreflective materials, and conspicuity, is reviewed to evaluate the effectiveness of chromaticity specifications for highway signs and markings. The current highway specifications are typically for colors of medium lightness and saturation, and sometimes can appear quite dark. Data from a study by Collins et al (1986) are reanalyzed to compare color appearance data for both ANSI and highway colors viewed under seven illuminants, including several HID sources. The analysis demonstrated that the ANSI colors, expecially safety yellow, were identified more accurately in terms of color name, lightness, saturation, and primary hue than the corresponding highway color. In addition, selected measurements of the chromaticity of retroreflective materials were made as background for assessing the feasibility of developing a nighttime chromaticity specification. Based on the analysis of the appearance of safety colors under different illuminants, it is recommended that consideration be given to switching the FHWA specifications for the chromaticity of colors used on traffic control devices to the ANSI specifications.

12. KEY WORDS (Six to twelve entries; alphabetical order: copitalize only proper nomes; and seporate key words by semicolons)

Chromaticity, CIELAB, color, color code, color vision, color defects, conspicuity, highway, safety, retroreflectivity

13. AVAILABILITY

[] Unlimited

$\square$ For Official Distribution. Do Not Release to NTIS

$\square$ Order From Superintendent of Documents, U.S. Government Printing Office, Washington, D.C. 20402.

Order From National Technical Information Service (NTIS), Springfield, VA. 2216I
14. NO. OF PBINJED PAGES 109

15. Price

$\$ 18.95$ 
. 

